\title{
ACTUALIZACIÓN DEL CATÁLOGO DE LAS PLANTAS VASCULARES DEL CONO SUR
}

\author{
Fernando O. Zuloaga', Manuel J. Belgrano'1 \& Christian A. Zanotti²
}

\author{
${ }^{1}$ Instituto de Botánica Darwinion (CONICET-ANCEFN), Labardén 200, Casilla de Correo 22, B1642HYD, San \\ Isidro, Buenos Aires, Argentina; fzuloaga@darwin.edu.ar (autor corresponsal). \\ ${ }^{2}$ Instituto de Limnología "Dr. Raúl A. Ringuelet" (CONICET), Bv. 120 y 62, 1900 La Plata, Buenos Aires, Argentina.
}

\begin{abstract}
Zuloaga, F. O.; M. J. Belgrano \& C. A. Zanotti. 2019. An update of the Catalogue of the Vascular Plants of the Southern Cone. Darwiniana, nueva serie 7(2): 208-278.

An update of the "Catalogue of the vascular plants of the Southern Cone" of South America (Argentina, southern Brazil, Chile, Paraguay, and Uruguay) is here summarized, eleven years after the publication of the original treatment. A summary of all Lycophyta, Monilophyta, Gimnospermae, and Angiospermae (Monocotyledoneae and Dictotyledoneae), updated to July 2019, is here presented. This checklist includes nowadays 289 families, 2813 genera, and 18931 species. The number of species is 1238 higher than that registered for the original version, which shows the large number of floristic and taxonomic news published for the area, and the importance of keeping this information updated. Tables sumarizing the floristic information for the Southern Cone and for each country are included, distinguishing by higher groups, families and genera; a comparison with the original version of the catalogue is conducted; endemic families and genera are specified, as well as the introduced ones; finally floristic relationships between the involved countries are evaluated. In addition, two digital appendixes are included, one with the new version of the catalogue, the other with a summary of the flora for each country.
\end{abstract}

Keywords. Argentina; checklist; Chile; Paraguay; southern Brazil; southern Cone; Uruguay; vascular plants.

Resumen. Zuloaga, F. O.; M. J. Belgrano \& C. A. Zanotti. 2019. Actualización del Catálogo de las Plantas Vasculares del Cono Sur. Darwiniana, nueva serie 7(2): 208-278.

En la presente contribución se brinda una versión actualizada del "Catálogo de las plantas vasculares del Cono Sur" de Sudamérica (Argentina, sur del Brasil, Chile, Paraguay y Uruguay), once años después de la publicación de su versión original. Se brinda un detalle de las Lycophyta, Monilophyta, Gymnospermae y Angiospermae (Monocoyledoneae y Dicotyledoneae) registradas para el área hasta julio de 2019. Este catálogo incluye, en la actualidad, 289 familias, 2813 géneros y 18931 especies. Este número de especies es superior en 1238 al registrado en la versión original, lo cual ilustra sobre la gran cantidad de novedades florísticas y taxonómicas registradas para el área y la importancia de matener esta información actualizada. Es digno de destacar que más del 40\% de las especies presentes en el Cono Sur son endémicas de esta área. En este trabajo se aportan tablas que resumen la información florística para el Cono Sur y para cada uno de los países que lo integran, discriminando por grandes grupos taxonómicos, familias y géneros; se hacen comparaciones respecto a la versión original, se especifican las familias y géneros endémicos del área y también aquellos introducidos; finalmente se analizan las relaciones florísticas entre los países involucrados. Adicionalmente, se incluyen apéndices en formato digital, que corresponden a la nueva versión catálogo, completo y actualizado, y al resumen florístico para cada país.

Palabras clave. Argentina; catálogo; Chile; Cono Sur; Paraguay; plantas vasculares; sur del Brasil; Uruguay. 


\section{INTRODUCCIÓN}

La confección del Catálogo de plantas vasculares del Cono Sur surgió luego de un proyecto previo, el "Catálogo de las plantas vasculares de Argentina". Este proyecto se gestó a comienzos de la década de 1990, a partir de la colaboración entre el Instituto de Botánica Darwinion y el Missouri Botanical Garden, a través de su director, el Dr. Peter H. Raven. De este modo se inició, con las dificultades propias de llevar a cabo un trabajo de esta complejidad hace casi treinta años, el análisis y la informatización del fichero de plantas existente en el Darwinion. Este fichero compendiaba datos sobre especies, con sus nombres vigentes y sinónimos, trabajo llevado a cabo durante muchos años por diversos investigadores de este instituto.

Inicialmente, esta información fue digitalizada en archivos de Microsoft Word, por no contar con una estructura de base de datos (en su momento se llevó a cabo una experiencia fallida con pcTROPICOS, herramienta brindada por el Jardín Botánico de Missouri). Es así que las dos primeras entregas del Catálogo de Plantas Vasculares de la Argentina se compilaron en su totalidad mediante este procesador de textos (Zuloaga et al. 1994, referida a la familia Poaceae; Zuloaga \& Morrone, 1996, Pteridófitas, Gimnospermas y Angiospermas Monocotiledóneas). Posteriormente, el Ing. Daniel Rodríguez comenzó a desarrollar una base de datos en Microsoft Access, que fue pacientemente mejorada y ampliada en conjunción con investigadores del Instituto. Así, la tercera entrega del Catálogo de Argentina (Zuloaga \& Morrone, 1999, Angiospermas Dicotiledóneas), publicada en dos tomos, se basó mayoritamente en los datos almacenados en esta base de datos.

Como se comentó inicialmente, la fructífera, aunque siempre mejorable, experiencia con el Catálogo de plantas vasculares de la Argentina nos condujo hacia un proyecto más ambicioso, la preparación de un Catálogo que incluyera las plantas vasculares de América del Sur templada y subtropical, el denominado "Cono Sur" de Sudamérica, que abarca los territorios de Argentina, Chile, Paraguay, Uruguay y el sur de Brasil (estados de Paraná, Rio Grande do Sul y Santa Catarina). Este nuevo emprendimiento requirió la migración de la base de datos desde Microsoft Access a MySQL, con dos objetivos primordiales: 1) que la misma permitiera el incremento, almacenamiento y manejo de grandes cantidades de datos, referidos a nombres científicos (aceptados y sinónimos), distribución geográfica, colecciones de herbario, botánicos, siglas de autoridades, etc. (ver "Materiales y métodos"); 2) que fuera accesible no sólo internamente, es decir, para el personal del instituto, sino también para el público en general, a través de internet. Esta nueva base de datos de desarrollo web, y que denominamos Documenta Florae Australis ${ }^{\mathcal{O}}$ (en adelante DFA), fue desarrollada en colaboración con el Instituto de Botánica del Nordeste (IBONE, CONICET, Corrientes) y el Instituto Multidisciplinario de Biología Vegetal (IMBIV, CONICET, Córdoba), y constituyó la base de la publicación del Catálogo del Cono Sur (Zuloaga et al., 2008).

De este modo, los trabajos realizados y la enorme cantidad de datos disponibles en la base de datos DFA constituyen un valioso repositorio digital, de libre acceso, sobre la biodiversidad de plantas vasculares del Cono Sur. Los objetivos particulares que se persiguen mediante el desarrollo e implementación de esta base de datos son, entonces: 1) reunir toda la información disponible para cada taxón y establecer un sistema dinámico de actualización; 2) permitir el libre acceso a esta información, a través de la página web del catálogo del Cono Sur (http:// conosur.floraargentina.edu.ar); 3) utilizar estas herramientas para orientar políticas de protección de especies nativas en peligro y colaborar en este sentido con la conservación de la biodiversidad.

Desde la publicación original del Catálogo del Cono Sur (Zuloaga et al., 2008), su información florística ha sido constantemente actualizada y atendidas las novedades sistemáticas, taxonómicas y nomenclaturales. Con anterioridad al presente trabajo, la información contenida en DFA se utilizó en otras colaboraciones florísticas (Zuloaga \& Belgrano, 2015; Ulloa et al., 2017) y hoy, habiendo transcurrido once años desde la publicación original del Catálogo del Cono Sur, el objetivo principal de 
este trabajo es proveer de una nueva actualización, comparar los datos de aquella publicación con los disponibles actualmente $\mathrm{y}$, finalmente, la de brindar una nueva versión del catálogo completo, en esta oportunidad en soporte digital. (Apéndice 1 disponible en: http://www.ojs.darwin.edu.ar/index. $\mathrm{php} /$ darwiniana/article/view/861/1167).

\section{MATERIALES Y MÉTODOS}

Los tratamientos sistemáticos para cada familia fueron preparados oportunamente por especialistas (cfr. Zuloaga et al., 2008) y actualizados desde entonces por los editores del Catálogo del Cono Sur, sobre la base de nuevas publicaciones referidas a las plantas vasculares del área. Esta información, antes de ser incorporada, es revisada y analizada cuidadosamente; en muchos casos, antes de tomar alguna decisión, se consulta previamente con especialistas. Estos trabajos conllevan la adición de nuevos taxones para el Cono Sur, extensiones de área, nueva sinonimia, etc. Recientemente, se consultaron el Catálogo de las plantas vasculares de Chile (Rodríguez et al., 2018) y la Lista de Flora de Brasil (BFG, 2018, 2020).

Adicionalmente, gran cantidad de especímenes de herbario, depositados en los principales herbarios de la región y del mundo, han sido y son revisados por especialistas e incorporados a DFA, suplementando así los datos publicados en diferentes revistas científicas.

Así, la base de datos DFA, con la información permanentemente actualizada, provee de una lista al día de las plantas vasculares del Cono Sur. Los datos pueden visualizarse on-line en: http:// conosur.floraargentina.edu.ar

El catálogo de plantas vasculares del Cono Sur sigue, en líneas generales, el ordenamiento filogenético publicado por «Angiosperm Phylogeny Group» (APG IV - Byng et al., 2016). Sólo en casos puntuales no se ha seguido este ordenamiento, como por ejemplo para las familias Chenopodiaceae, Malesherbiaceae y Turneraceae, a las que se consideran separadamente.
En el Catálogo actualizado, que se presenta como Apéndice (Apéndice 1 disponible en: http:// www.ojs.darwin.edu.ar/index.php/darwiniana/ article/view/861/1167), la información que se brinda para cada especie o taxón infraespecífico, comprende el nombre de uso correcto, sinónimos relevantes para el área, distribución geográfica por país y por divisiones menores (provincias, departamentos, estados), status y elevación, de acuerdo al siguiente formato:

1) Nombre aceptado, corresponde al nombre de uso correcto (de acuerdo con el Código Internacional de Nomenclatura Botánica - Turland et al., 2018), se escribe en negritas y los autores de los taxones se abrevian de acuerdo a Brummitt \& Powell (1992).

2) Sinónimos, se mencionan luego del nombre aceptado y se escriben en cursiva; se incluyen sólo los sinónimos, tanto taxonómicos como nomenclaturales, pertenecientes al área de estudio.

3) Hábito, status y elevación. Se indica si se trata de árboles, arbustos o hierbas; si son nativos, endémicos o introducidos en el área; cuando es posible, se indica el rango altitudinal en el que crece el taxón.

4) Distribución. Indica la presencia de los taxones en los distintos países del Cono Sur, discriminando según las divisiones políticas de primer grado para cada país, utilizando las siguientes abreviaturas:

Argentina: BAI: Buenos Aires; CAT: Catamarca; DFE: Ciudad Autónoma de Buenos Aires. CHA: Chaco; CHU: Chubut; COR: Córdoba; COS: Corrientes; ERI: Entre Ríos; FOR: Formosa; JUJ: Jujuy; LPA: La Pampa; LRI: La Rioja; MEN: Mendoza; MIS: Misiones; NEU: Neuquén; RNE: Río Negro; SAL: Salta; SCR: Santa Cruz; SDE: Santiago del Estero; SFE: Santa Fe; SJU: San Juan; SLU: San Luis; TDF: Tierra del Fuego, Antártida e Islas del Atlántico Sur; TUC: Tucumán.

Sur de Brasil: PAR: Paraná; RGS: Rio Grande do Sul; SCA: Santa Catarina.

Chile: AIS: Aisen; ANT: Antofagasta; ARA: Araucanía: AYP: Arica y Parinacota; ATA: Atacama; BIO: Bio Bío; COQ: Coquimbo; IDP: Isla de Pascua; IDE: Islas Desventuradas; JFE: Islas Juan Fernández; LBO: Libertador Bernardo O'Higgins; LLA: Los Lagos; LRI: Los Ríos; MAG: Magallanes; MAU: Maule; RME: Metropolitana; TAR: Tarapacá; VAL: Valparaíso. 
Paraguay: APA: Alto Paraná; APY: Alto Paraguay (incluye Boquerón, Chaco y Nueva Asunción); AMA: Amambay; CAA: Caazapá; CAU: Caaguazú; CAN: Canindeyú; CEN: Central; CON: Concepción; COA: Cordillera; GUA: Guairá; ITA: Itapúa; MIE: Misiones; ÑEE: Ñeembucú; PAI: Paraguarí; PHA: Presidente Hayes; SPE: San Pedro.

Uruguay: ART: Artigas; CAS: Canelones; CLA: Cerro Largo; COL: Colonia; DUR: Durazno; FLO: Flores; FLA: Florida; LAV: Lavalleja; MAL: Maldonado; MON: Montevideo; PAY: Paysandú; RNO: Río Negro; RIV: Rivera; ROC: Rocha; SAO: Salto; SJO: San José; SOR: Soriano; TAC: Tacuarembó; TYT: Treinta y Tres.

\section{RESULTADOS Y DISCUSIÓN}

\section{Nueva versión del Catálogo del Cono Sur}

En el Apéndice 1 se presenta el nuevo Catálogo de las plantas vasculares del Cono Sur, como texto digitalizado en formato PDF, siguiendo el formato antes mencionado en "Materiales y métodos".
Las familias se presentan alfabéticamente, primero las Lycophyta y Monilophyta, luego las Gimnospermae y por último las Angiospermae. (Apéndice 1 disponible en: http://www.ojs.darwin. edu.ar/index.php/darwiniana/article/view/861/1167)

La información para cada taxón puede también consultarse on-line en http://conosur. floraargentina.edu.ar, accediendo por nombre científico. A través de esta página web se pueden visualizar, adicionalmente, ilustraciones botánicas y fotografías de campo para la mayoría de los taxones. En la actualidad están disponibles más de 12.000 ilustraciones originales y más de 45.000 fotografías.

\section{Análisis de la Flora del Cono Sur}

En cuanto a la Flora vascular del Cono Sur, en la Tabla 1 se presenta un resumen, actualizado a julio de 2019. Para cada gran grupo taxonómico se indica el número de familias, géneros, especies y especies endémicas, y se indica el porcentaje con el que aporta cada uno al total de la flora.

Tabla 1. Resumen de la Flora vascular del Cono Sur (2019).

\begin{tabular}{|l|c|c|c|c|c|c|}
\hline Grupo & Familias & Géneros & Especies & \% & Endémicas & \% \\
\hline Lycophyta/Monilophyta & 37 & 156 & 890 & 4,70 & 172 & 2,23 \\
\hline Gymnospermae & 5 & 13 & 40 & 0,21 & 16 & 0,21 \\
\hline Angiosp.-Dicotyledoneae & 202 & 2028 & 13374 & 70,65 & 5786 & 74,99 \\
\hline Angiosp,-Monocotyledoneae & 45 & 616 & 4627 & 24,46 & 1739 & 22,60 \\
\hline Total & $\mathbf{2 8 9}$ & $\mathbf{2 8 1 3}$ & $\mathbf{1 8 9 3 1}$ & & $\mathbf{7 7 1 3}$ & \\
\hline
\end{tabular}

Para poder establecer comparaciones, en la Tabla 2 se presenta el resumen de la Flora Vascular del Cono Sur publicada en su versión impresa original (Zuloaga et al., 2008).

Tabla 2. Resumen de la Flora vascular del Cono Sur (2008).

\begin{tabular}{|l|c|c|c|c|c|c|}
\hline Grupo & Familias & Géneros & Especies & \% & Endémicas & \% \\
\hline Lycophyta/Monilophyta & 29 & 118 & 759 & 4,29 & 164 & 2,13 \\
\hline Gymnospermae & 5 & 12 & 30 & 0,17 & 17 & 0,22 \\
\hline Angiosp.-Dicotyledoneae & 216 & 1846 & 12551 & 70,94 & 5828 & 75,78 \\
\hline Angiosp.-Monocotyledoneae & 58 & 610 & 4353 & 24,60 & 1682 & 21,87 \\
\hline Total & $\mathbf{3 0 8}$ & $\mathbf{2 5 8 6}$ & $\mathbf{1 7 6 9 3}$ & & $\mathbf{7 6 9 1}$ & \\
\hline
\end{tabular}


De la comparación entre estas tablas (Tablas 1 y 2) se evidencian difrencias significativas en los rangos de familia, género y especie. En la actualidad se contabilizan 19 familias menos, 227 géneros más y 1238 especies más que en 2008, lo que representa un incremento cercano al $7 \%$ en el número de especies citadas; a su vez, se contabilizaron 22 especies endémicas menos.
Un detalle de las familias que se registran en el Cono Sur, con su número de géneros, especies, taxones infraespecíficos, especies endémicas, nativas e introducidas, se brinda en la Tabla 3. Aquí se contabilizan 289 familias, 2813 géneros, 18931 especies (de las cuales 7713 son endémicas del Cono Sur, 9892 nativas y 1326 introducidas), con 1713 taxones de rango infraespecífico.

Tabla 3. Familias de plantas vasculares representadas en el Cono Sur (incluyendo número de géneros, especies, taxones infraespecíficos, especies endémicas, nativas e introducidas).

\begin{tabular}{|c|c|c|c|c|c|c|c|}
\hline Grupo & Familia & Géneros & Especies & Infraesp. & Endémicas & Nativas & Introd. \\
\hline Angiosp.-Dicot. & Acanthaceae & 23 & 134 & 4 & 28 & 99 & 7 \\
\hline Angiosp.-Dicot. & Aceraceae & 1 & 2 & & & & 2 \\
\hline Angiosp.-Dicot. & Achariaceae & 1 & 1 & & & & 1 \\
\hline Angiosp.-Dicot. & Achatocarpaceae & 1 & 5 & 3 & 2 & 3 & \\
\hline Angiosp.-Dicot. & Aextoxicaceae & 1 & 1 & & 1 & & \\
\hline Angiosp.-Dicot. & Aizoaceae & 8 & 21 & & 9 & 3 & 9 \\
\hline Angiosp.-Monocot. & Alismataceae & 6 & 24 & 1 & 3 & 19 & 2 \\
\hline Angiosp.-Monocot. & Alstroemeriaceae & 3 & 62 & 17 & 48 & 14 & \\
\hline Angiosp.-Dicot. & Amaranthaceae & 15 & 143 & 50 & 42 & 89 & 12 \\
\hline Angiosp.-Monocot. & Amaryllidaceae & 33 & 295 & 10 & 246 & 36 & 13 \\
\hline Angiosp.-Dicot. & Anacampserotaceae & 2 & 3 & & 2 & 1 & \\
\hline Angiosp.-Dicot. & Anacardiaceae & 12 & 52 & 14 & 17 & 34 & 1 \\
\hline Lyco/Monilophyta & Anemiaceae & 1 & 15 & 4 & & 15 & \\
\hline Angiosp.-Dicot. & Annonaceae & 6 & 31 & & 5 & 26 & \\
\hline Angiosp.-Dicot. & Apiaceae & 43 & 207 & 4 & 128 & 53 & 26 \\
\hline Angiosp.-Dicot. & Apocynaceae & 62 & 348 & 12 & 129 & 212 & 7 \\
\hline Angiosp.-Dicot. & Apodanthaceae & 1 & 2 & & & 2 & \\
\hline Angiosp.-Dicot. & Aponogetonaceae & 1 & 1 & & & & 1 \\
\hline Angiosp.-Dicot. & Aquifoliaceae & 1 & 12 & 2 & 1 & 11 & \\
\hline Angiosp.-Monocot. & Araceae & 29 & 84 & 1 & 12 & 61 & 11 \\
\hline Angiosp.-Dicot. & Araliaceae & 8 & 36 & & 7 & 27 & 2 \\
\hline Gymnospermae & Araucariaceae & 1 & 2 & & 1 & 1 & \\
\hline Angiosp.-Monocot. & Arecaceae & 15 & 53 & 3 & 15 & 35 & 3 \\
\hline Angiosp.-Dicot. & Aristolochiaceae & 3 & 40 & 1 & 13 & 27 & \\
\hline Angiosp.-Monocot. & Asparagaceae & 14 & 34 & 1 & 11 & 10 & 13 \\
\hline Angiosp.-Monocot. & Asphodelaceae & 3 & 3 & & & 1 & 2 \\
\hline Lyco/Monilophyta & Aspleniaceae & 4 & 67 & 2 & 10 & 55 & 2 \\
\hline Angiosp.-Monocot. & Asteliaceae & 1 & 1 & & 1 & & \\
\hline Angiosp.-Dicot. & Asteraceae & 352 & 2593 & 235 & 1404 & 1037 & 152 \\
\hline
\end{tabular}


Tabla 3. (Continuación).

\begin{tabular}{|c|c|c|c|c|c|c|c|}
\hline Grupo & Familia & Géneros & Especies & Infraesp. & Endémicas & Nativas & Introd. \\
\hline Angiosp.-Dicot. & Atherospermataceae & 1 & 1 & & 1 & & \\
\hline Lyco/Monilophyta & Athyriaceae & 3 & 24 & & 1 & 22 & 1 \\
\hline Angiosp.-Dicot. & Balanophoraceae & 5 & 7 & 1 & & 7 & \\
\hline Angiosp.-Dicot. & Balsaminaceae & 1 & 3 & & & & 3 \\
\hline Angiosp.-Dicot. & Basellaceae & 2 & 5 & 1 & & 5 & \\
\hline Angiosp.-Dicot. & Begoniaceae & 1 & 61 & 1 & 33 & 28 & \\
\hline Angiosp.-Dicot. & Berberidaceae & 1 & 36 & 1 & 31 & 5 & \\
\hline Angiosp.-Dicot. & Berberidopsidaceae & 1 & 1 & & 1 & & \\
\hline Angiosp.-Dicot. & Betulaceae & 2 & 5 & & & 1 & 4 \\
\hline Angiosp.-Dicot. & Bignoniaceae & 28 & 123 & 4 & 19 & 101 & 3 \\
\hline Angiosp.-Dicot. & Bixaceae & 2 & 3 & & & 3 & \\
\hline Lyco/Monilophyta & Blechnaceae & 10 & 48 & 1 & 17 & 31 & \\
\hline Angiosp.-Dicot. & Boraginaceae & 34 & 222 & 11 & 107 & 90 & 25 \\
\hline Angiosp.-Dicot. & Brassicaceae & 71 & 298 & 4 & 176 & 51 & 71 \\
\hline Angiosp.-Monocot. & Bromeliaceae & 25 & 323 & 57 & 151 & 172 & \\
\hline Angiosp.-Monocot. & Burmanniaceae & 6 & 9 & 1 & & 9 & \\
\hline Angiosp.-Dicot. & Burseraceae & 2 & 3 & & & 3 & \\
\hline Angiosp.-Dicot. & Cabombaceae & 1 & 3 & & & 3 & \\
\hline Angiosp.-Dicot. & Cactaceae & 56 & 437 & 90 & 311 & 120 & 6 \\
\hline Angiosp.-Dicot. & Calceolariaceae & 2 & 84 & 20 & 65 & 19 & \\
\hline Angiosp.-Dicot. & Calophyllaceae & 2 & 3 & 2 & & 3 & \\
\hline Angiosp.-Dicot. & Calyceraceae & 5 & 47 & 3 & 42 & 5 & \\
\hline Angiosp.-Dicot. & Campanulaceae & 10 & 62 & 9 & 23 & 33 & 6 \\
\hline Angiosp.-Dicot. & Canellaceae & 1 & 2 & & 1 & 1 & \\
\hline Angiosp.-Dicot. & Cannabaceae & 3 & 8 & 1 & & 6 & 2 \\
\hline Angiosp.-Monocot. & Cannaceae & 1 & 5 & & 1 & 4 & \\
\hline Angiosp.-Dicot. & Capparaceae & 8 & 13 & & & 13 & \\
\hline Angiosp.-Dicot. & Caprifoliaceae & 9 & 97 & 1 & 68 & 17 & 12 \\
\hline Angiosp.-Dicot. & Cardiopteridaceae & 1 & 6 & & 1 & 5 & \\
\hline Angiosp.-Dicot. & Caricaceae & 3 & 6 & & 1 & 5 & \\
\hline Angiosp.-Dicot. & Caryocaraceae & 1 & 1 & 1 & & 1 & \\
\hline Angiosp.-Dicot. & Caryophyllaceae & 30 & 175 & 32 & 81 & 51 & 43 \\
\hline Angiosp.-Dicot. & Celastraceae & 15 & 41 & & 5 & 36 & \\
\hline Angiosp.-Dicot. & Ceratophyllaceae & 1 & 2 & 1 & & 2 & \\
\hline Angiosp.-Dicot. & Cervantesiaceae & 2 & 3 & & 1 & 2 & \\
\hline Angiosp.-Dicot. & Chenopodiaceae & 17 & 124 & 10 & 60 & 30 & 34 \\
\hline Angiosp.-Dicot. & Chrysobalanaceae & 3 & 5 & & & 5 & \\
\hline Angiosp.-Dicot. & Cistaceae & 1 & 1 & & 1 & & \\
\hline Angiosp.-Dicot. & Cleomaceae & 4 & 19 & 1 & 4 & 15 & \\
\hline
\end{tabular}


Tabla 3. (Continuación).

\begin{tabular}{|c|c|c|c|c|c|c|c|}
\hline Grupo & Familia & Géneros & Especies & Infraesp. & Endémicas & Nativas & Introd. \\
\hline Angiosp.-Dicot. & Clethraceae & 1 & 2 & 2 & 1 & 1 & \\
\hline Angiosp.-Dicot. & Clusiaceae & 3 & 5 & 1 & & 5 & \\
\hline Angiosp.-Dicot. & Columelliaceae & 1 & 1 & 1 & 1 & & \\
\hline Angiosp.-Dicot. & Combretaceae & 5 & 14 & & 2 & 11 & 1 \\
\hline Angiosp.-Monocot. & Commelinaceae & 9 & 44 & & 9 & 33 & 2 \\
\hline Angiosp.-Dicot. & Connaraceae & 2 & 3 & & & 3 & \\
\hline Angiosp.-Dicot. & Convolvulaceae & 15 & 226 & 45 & 70 & 146 & 10 \\
\hline Angiosp.-Dicot. & Coriariaceae & 1 & 1 & & 1 & & \\
\hline Angiosp.-Dicot. & Corsiaceae & 1 & 1 & & 1 & & \\
\hline Angiosp.-Monocot. & Costaceae & 1 & 3 & & & 3 & \\
\hline Angiosp.-Dicot. & Crassulaceae & 5 & 24 & 2 & 5 & 10 & 9 \\
\hline Angiosp.-Dicot. & Cucurbitaceae & 24 & 83 & 8 & 14 & 58 & 11 \\
\hline Lyco/Monilophyta & Culcitaceae & 1 & 1 & & & 1 & \\
\hline Angiosp.-Dicot. & Cunoniaceae & 4 & 12 & & 4 & 8 & \\
\hline Gymnospermae & Cupressaceae & 5 & 6 & & 3 & & 3 \\
\hline Lyco/Monilophyta & Cyatheaceae & 2 & 24 & 1 & & 23 & 1 \\
\hline Angiosp.-Monocot. & Cyclanthaceae & 1 & 2 & & 1 & 1 & \\
\hline Angiosp.-Monocot. & Cymodoceaceae & 1 & 1 & & & 1 & \\
\hline Angiosp.-Monocot. & Cyperaceae & 34 & 516 & 76 & 163 & 334 & 19 \\
\hline Lyco/Monilophyta & Cystopteridaceae & 1 & 2 & & & 2 & \\
\hline Lyco/Monilophyta & Dennstaedtiaceae & 7 & 20 & 8 & & 20 & \\
\hline Lyco/Monilophyta & Dicksoniaceae & 3 & 5 & & 3 & 2 & \\
\hline Lyco/Monilophyta & Didymochlaenaceae & 1 & 1 & & & 1 & \\
\hline Angiosp.-Dicot. & Dilleniaceae & 4 & 12 & & & 12 & \\
\hline Angiosp.-Monocot. & Dioscoreaceae & 1 & 101 & 6 & 56 & 44 & 1 \\
\hline Angiosp.-Dicot. & Droseraceae & 1 & 6 & & 1 & 5 & \\
\hline Lyco/Monilophyta & Dryopteridaceae & 16 & 123 & 2 & 32 & 88 & 3 \\
\hline Angiosp.-Dicot. & Ebenaceae & 1 & 3 & & & 3 & \\
\hline Angiosp.-Dicot. & Elaeagnaceae & 1 & 1 & & & & 1 \\
\hline Angiosp.-Dicot. & Elaeocarpaceae & 4 & 11 & 1 & 5 & 6 & \\
\hline Angiosp.-Dicot. & Elatinaceae & 1 & 3 & 1 & 1 & 2 & \\
\hline Gymnospermae & Ephedraceae & 1 & 9 & & 7 & 2 & \\
\hline Lyco/Monilophyta & Equisetaceae & 1 & 2 & & & 2 & \\
\hline Angiosp.-Dicot. & Eremolepidaceae & 3 & 3 & & 2 & 1 & \\
\hline Angiosp.-Dicot. & Ericaceae & 7 & 43 & 3 & 23 & 20 & \\
\hline Angiosp.-Monocot. & Eriocaulaceae & 6 & 44 & 5 & 18 & 26 & \\
\hline Angiosp.-Dicot. & Erythroxylaceae & 1 & 19 & & 4 & 15 & \\
\hline Angiosp.-Dicot. & Escalloniaceae & 3 & 31 & 6 & 20 & 11 & \\
\hline Angiosp.-Dicot. & Euphorbiaceae & 42 & 453 & 38 & 188 & 240 & 25 \\
\hline
\end{tabular}


Tabla 3. (Continuación).

\begin{tabular}{|c|c|c|c|c|c|c|c|}
\hline Grupo & Familia & Géneros & Especies & Infraesp. & Endémicas & Nativas & Introd. \\
\hline Angiosp.-Dicot. & Fabaceae & 183 & 1437 & 234 & 632 & 682 & 123 \\
\hline Angiosp.-Dicot. & Fagaceae & 1 & 1 & & & & 1 \\
\hline Angiosp.-Dicot. & Francoaceae & 7 & 18 & 3 & 15 & 3 & \\
\hline Angiosp.-Dicot. & Frankeniaceae & 1 & 10 & & 6 & 3 & 1 \\
\hline Angiosp.-Dicot. & Gentianaceae & 18 & 63 & 3 & 21 & 37 & 5 \\
\hline Angiosp.-Dicot. & Geraniaceae & 3 & 28 & 1 & 10 & 7 & 11 \\
\hline Angiosp.-Dicot. & Gesneriaceae & 10 & 49 & & 18 & 31 & \\
\hline Lyco/Monilophyta & Gleicheniaceae & 3 & 17 & 1 & 4 & 13 & \\
\hline Angiosp.-Dicot. & Gomortegaceae & 1 & 1 & & 1 & & \\
\hline Angiosp.-Dicot. & Goodeniaceae & 2 & 2 & & & 2 & \\
\hline Angiosp.-Dicot. & Griseliniaceae & 1 & 5 & 1 & 4 & 1 & \\
\hline Angiosp.-Dicot. & Grossulariaceae & 1 & 10 & 1 & 8 & 2 & \\
\hline Angiosp.-Dicot. & Gunneraceae & 1 & 11 & 2 & 7 & 4 & \\
\hline Angiosp.-Dicot. & Halophytaceae & 1 & 1 & & 1 & & \\
\hline Angiosp.-Dicot. & Haloragaceae & 4 & 6 & 3 & 3 & 3 & \\
\hline Angiosp.-Monocot. & Heliconiaceae & 1 & 5 & 1 & & 5 & \\
\hline Lyco/Monilophyta & Hemidictyaceae & 1 & 1 & & & 1 & \\
\hline Angiosp.-Dicot. & Humiriaceae & 1 & 1 & & & 1 & \\
\hline Angiosp.-Dicot. & Hydrangeaceae & 1 & 1 & & 1 & & \\
\hline Angiosp.-Monocot. & Hydrocharitaceae & 6 & 11 & & & 11 & \\
\hline Angiosp.-Dicot. & Hydroleaceae & 1 & 2 & 1 & & 2 & \\
\hline Lyco/Monilophyta & Hymenophyllaceae & 7 & 69 & 2 & 22 & 47 & \\
\hline Angiosp.-Dicot. & Hypericaceae & 1 & 31 & 7 & 17 & 11 & 3 \\
\hline Angiosp.-Monocot. & Hypoxidaceae & 3 & 6 & & 2 & 3 & 1 \\
\hline Angiosp.-Dicot. & Icacinaceae & 1 & 1 & & 1 & & \\
\hline Angiosp.-Monocot. & Iridaceae & 29 & 229 & 31 & 149 & 64 & 16 \\
\hline Lyco/Monilophyta & Isoetaceae & 1 & 21 & & 17 & 4 & \\
\hline Angiosp.-Dicot. & Juglandaceae & 2 & 2 & & & 1 & 1 \\
\hline Angiosp.-Monocot. & Juncaceae & 7 & 68 & 8 & 27 & 34 & 7 \\
\hline Angiosp.-Monocot. & Juncaginaceae & 2 & 6 & & 1 & 5 & \\
\hline Angiosp.-Dicot. & Krameriaceae & 1 & 3 & & 1 & 2 & \\
\hline Angiosp.-Dicot. & Lacistemataceae & 1 & 2 & & & 2 & \\
\hline Angiosp.-Dicot. & Lamiaceae & 49 & 240 & 14 & 76 & 128 & 36 \\
\hline Angiosp.-Dicot. & Lardizabalaceae & 2 & 2 & & 2 & & \\
\hline Angiosp.-Dicot. & Lauraceae & 13 & 90 & & 13 & 74 & 3 \\
\hline Angiosp.-Dicot. & Lecythidaceae & 1 & 2 & & & 2 & \\
\hline Angiosp.-Dicot. & Lentibulariaceae & 3 & 28 & & 4 & 24 & \\
\hline Angiosp.-Monocot. & Liliaceae & 1 & 1 & & & & 1 \\
\hline Angiosp.-Dicot. & Linaceae & 2 & 16 & 2 & 5 & 8 & 3 \\
\hline
\end{tabular}


Tabla 3. (Continuación).

\begin{tabular}{|c|c|c|c|c|c|c|c|}
\hline Grupo & Familia & Géneros & Especies & Infraesp. & Endémicas & Nativas & Introd. \\
\hline Angiosp.-Dicot. & Linderniaceae & 3 & 4 & & & 3 & 1 \\
\hline Lyco/Monilophyta & Lindsaeaceae & 1 & 10 & 3 & & 10 & \\
\hline Angiosp.-Dicot. & Loasaceae & 10 & 73 & 4 & 53 & 19 & 1 \\
\hline Angiosp.-Dicot. & Loganiaceae & 2 & 30 & & 11 & 19 & \\
\hline Lyco/Monilophyta & Lomariopsidaceae & 1 & 1 & & & 1 & \\
\hline Angiosp.-Dicot. & Loranthaceae & 7 & 15 & & 6 & 9 & \\
\hline Lyco/Monilophyta & Lycopodiaceae & 9 & 45 & & 14 & 31 & \\
\hline Lyco/Monilophyta & Lygodiaceae & 1 & 2 & & & 2 & \\
\hline Angiosp.-Dicot. & Lythraceae & 8 & 66 & 9 & 15 & 45 & 6 \\
\hline Angiosp.-Dicot. & Magnoliaceae & 1 & 1 & & & 1 & \\
\hline Angiosp.-Dicot. & Malesherbiaceae & 1 & 16 & 9 & 15 & 1 & \\
\hline Angiosp.-Dicot. & Malpighiaceae & 30 & 129 & & 25 & 104 & \\
\hline Angiosp.-Dicot. & Malvaceae & 63 & 533 & 30 & 202 & 315 & 16 \\
\hline Angiosp.-Monocot. & Marantaceae & 6 & 32 & & 6 & 26 & \\
\hline Lyco/Monilophyta & Marattiaceae & 3 & 5 & & & 5 & \\
\hline Angiosp.-Dicot. & Marcgraviaceae & 3 & 4 & 1 & & 4 & \\
\hline Lyco/Monilophyta & Marsileaceae & 3 & 6 & & 1 & 5 & \\
\hline Angiosp.-Dicot. & Martyniaceae & 2 & 3 & & 1 & 2 & \\
\hline Angiosp.-Monocot. & Mayacaceae & 1 & 3 & & & 3 & \\
\hline Angiosp.-Dicot. & Mazaceae & 1 & 1 & & & & 1 \\
\hline Angiosp.-Dicot. & Melastomataceae & 28 & 233 & 6 & 48 & 184 & 1 \\
\hline Angiosp.-Dicot. & Meliaceae & 5 & 22 & 4 & 2 & 19 & 1 \\
\hline Angiosp.-Dicot. & Menispermaceae & 6 & 17 & 3 & 1 & 16 & \\
\hline Angiosp.-Dicot. & Menyanthaceae & 1 & 2 & & & 2 & \\
\hline Angiosp.-Dicot. & Microteaceae & 1 & 5 & & & 5 & \\
\hline Angiosp.-Dicot. & Misodendraceae & 1 & 8 & & 7 & 1 & \\
\hline Angiosp.-Dicot. & Molluginaceae & 2 & 4 & & & 2 & 2 \\
\hline Angiosp.-Dicot. & Monimiaceae & 4 & 18 & & 4 & 14 & \\
\hline Angiosp.-Dicot. & Montiaceae & 8 & 68 & & 59 & 8 & 1 \\
\hline Angiosp.-Dicot. & Moraceae & 9 & 46 & 3 & 1 & 42 & 3 \\
\hline Angiosp.-Dicot. & Muntingiaceae & 1 & 1 & & & 1 & \\
\hline Angiosp.-Monocot. & Musaceae & 1 & 2 & & & & 2 \\
\hline Angiosp.-Dicot. & Myricaceae & 1 & 2 & & & 2 & \\
\hline Angiosp.-Dicot. & Myristicaceae & 1 & 1 & & & 1 & \\
\hline Angiosp.-Dicot. & Myrtaceae & 25 & 317 & 15 & 100 & 208 & 9 \\
\hline Angiosp.-Dicot. & Nanodeaceae & 1 & 1 & & 1 & & \\
\hline Lyco/Monilophyta & Nephrolepidaceae & 1 & 7 & & & 7 & \\
\hline Angiosp.-Dicot. & Nothofagaceae & 1 & 10 & & 10 & & \\
\hline Angiosp.-Dicot. & Nyctaginaceae & 10 & 37 & 4 & 6 & 28 & 3 \\
\hline
\end{tabular}


Tabla 3. (Continuación).

\begin{tabular}{|c|c|c|c|c|c|c|c|}
\hline Grupo & Familia & Géneros & Especies & Infraesp. & Endémicas & Nativas & Introd. \\
\hline Angiosp.-Monocot. & Nymphaeaceae & 3 & 10 & 1 & & 7 & 3 \\
\hline Angiosp.-Dicot. & Ochnaceae & 2 & 12 & & & 12 & \\
\hline Angiosp.-Dicot. & Olacaceae & 3 & 5 & 1 & & 5 & \\
\hline Angiosp.-Dicot. & Oleaceae & 7 & 19 & & 2 & 7 & 10 \\
\hline Lyco/Monilophyta & Oleandraceae & 2 & 2 & & 2 & & \\
\hline Angiosp.-Dicot. & Onagraceae & 7 & 101 & 15 & 31 & 58 & 12 \\
\hline Lyco/Monilophyta & Ophioglossaceae & 5 & 13 & & 3 & 10 & \\
\hline Angiosp.-Dicot. & Opiliaceae & 1 & 2 & & & 2 & \\
\hline Angiosp.-Monocot. & Orchidaceae & 133 & 983 & 21 & 251 & 730 & 2 \\
\hline Angiosp.-Dicot. & Orobanchaceae & 12 & 55 & 2 & 22 & 27 & 6 \\
\hline Lyco/Monilophyta & Osmundaceae & 2 & 3 & & & 3 & \\
\hline Angiosp.-Dicot. & Oxalidaceae & 1 & 122 & 19 & 71 & 49 & 2 \\
\hline Angiosp.-Dicot. & Papaveraceae & 7 & 22 & & 2 & 3 & 17 \\
\hline Angiosp.-Dicot. & Passifloraceae & 1 & 43 & 2 & 3 & 40 & \\
\hline Angiosp.-Dicot. & Pentaphylacaceae & 1 & 2 & & & 2 & \\
\hline Angiosp.-Dicot. & Peraceae & 1 & 2 & & & 2 & \\
\hline Angiosp.-Monocot. & Philesiaceae & 2 & 2 & & 2 & & \\
\hline Angiosp.-Dicot. & Phrymaceae & 1 & 5 & 2 & 4 & 1 & \\
\hline Angiosp.-Dicot. & Phyllanthaceae & 5 & 28 & 1 & 5 & 20 & 3 \\
\hline Angiosp.-Dicot. & Phytolaccaceae & 10 & 21 & 1 & 6 & 14 & 1 \\
\hline Angiosp.-Dicot. & Picramniaceae & 2 & 6 & & 1 & 5 & \\
\hline Angiosp.-Dicot. & Picrodendraceae & 1 & 1 & & & 1 & \\
\hline Gymnospermae & Pinaceae & 2 & 14 & & & & 14 \\
\hline Angiosp.-Dicot. & Piperaceae & 4 & 160 & 23 & 31 & 129 & \\
\hline Angiosp.-Dicot. & Pittosporaceae & 1 & 2 & & & & 2 \\
\hline Lyco/Monilophyta & Plagiogyriaceae & 1 & 1 & & & 1 & \\
\hline Angiosp.-Dicot. & Plantaginaceae & 30 & 164 & 20 & 68 & 68 & 28 \\
\hline Angiosp.-Dicot. & Plumbaginaceae & 5 & 7 & & 4 & 2 & 1 \\
\hline Angiosp.-Monocot. & Poaceae & 209 & 1563 & 190 & 544 & 800 & 219 \\
\hline Gymnospermae & Podocarpaceae & 4 & 9 & 1 & 5 & 4 & \\
\hline Angiosp.-Dicot. & Podostemaceae & 9 & 20 & & 8 & 12 & \\
\hline Angiosp.-Dicot. & Polemoniaceae & 10 & 14 & & 4 & 9 & 1 \\
\hline Angiosp.-Dicot. & Polygalaceae & 7 & 112 & 3 & 50 & 62 & \\
\hline Angiosp.-Dicot. & Polygonaceae & 16 & 104 & 6 & 40 & 36 & 28 \\
\hline Lyco/Monilophyta & Polypodiaceae & 22 & 99 & 3 & 10 & 89 & \\
\hline Angiosp.-Monocot. & Pontederiaceae & 2 & 14 & & 2 & 12 & \\
\hline Angiosp.-Dicot. & Portulacaceae & 2 & 30 & 7 & 15 & 13 & 2 \\
\hline Angiosp.-Monocot. & Potamogetonaceae & 3 & 14 & 1 & 2 & 11 & 1 \\
\hline Angiosp.-Dicot. & Primulaceae & 12 & 42 & 1 & 9 & 30 & 3 \\
\hline
\end{tabular}


Tabla 3. (Continuación).

\begin{tabular}{|c|c|c|c|c|c|c|c|}
\hline Grupo & Familia & Géneros & Especies & Infraesp. & Endémicas & Nativas & Introd. \\
\hline Angiosp.-Dicot. & Proteaceae & 7 & 14 & 3 & 6 & 7 & 1 \\
\hline Lyco/Monilophyta & Psilotaceae & 1 & 1 & & & 1 & \\
\hline Lyco/Monilophyta & Pteridaceae & 26 & 151 & 9 & 24 & 120 & 7 \\
\hline Angiosp.-Dicot. & Quiinaceae & 1 & 1 & & & 1 & \\
\hline Angiosp.-Dicot. & Quillajaceae & 1 & 2 & & 1 & 1 & \\
\hline Angiosp.-Dicot. & Ranunculaceae & 13 & 65 & 11 & 31 & 26 & 8 \\
\hline Angiosp.-Dicot. & Resedaceae & 1 & 5 & & & & 5 \\
\hline Angiosp.-Monocot. & Restionaceae & 2 & 2 & & 2 & & \\
\hline Angiosp.-Dicot. & Rhamnaceae & 17 & 42 & 3 & 20 & 20 & 2 \\
\hline Angiosp.-Dicot. & Rhizophoraceae & 1 & 1 & & & 1 & \\
\hline Angiosp.-Dicot. & Rosaceae & 23 & 133 & 6 & 49 & 37 & 47 \\
\hline Angiosp.-Dicot. & Rubiaceae & 60 & 321 & 14 & 82 & 227 & 12 \\
\hline Angiosp.-Monocot. & Ruppiaceae & 1 & 3 & 1 & & 3 & \\
\hline Angiosp.-Dicot. & Rutaceae & 13 & 34 & 3 & 4 & 25 & 5 \\
\hline Angiosp.-Dicot. & Sabiaceae & 1 & 1 & 3 & & 1 & \\
\hline Lyco/Monilophyta & Saccolomataceae & 1 & 3 & & & 3 & \\
\hline Angiosp.-Dicot. & Salicaceae & 9 & 47 & & 13 & 26 & 8 \\
\hline Lyco/Monilophyta & Salviniaceae & 2 & 7 & 1 & & 6 & 1 \\
\hline Angiosp.-Dicot. & Santalaceae & 1 & 1 & & 1 & & \\
\hline Angiosp.-Dicot. & Sapindaceae & 23 & 100 & 6 & 10 & 90 & \\
\hline Angiosp.-Dicot. & Sapotaceae & 7 & 27 & 1 & 2 & 25 & \\
\hline Angiosp.-Dicot. & Saxifragaceae & 5 & 7 & & 4 & 2 & 1 \\
\hline Lyco/Monilophyta & Schizaeaceae & 2 & 5 & & & 5 & \\
\hline Angiosp.-Dicot. & Schlegeliaceae & 1 & 1 & & 1 & & \\
\hline Angiosp.-Dicot. & Schoepfiaceae & 3 & 8 & 1 & 5 & 2 & 1 \\
\hline Angiosp.-Dicot. & Scrophulariaceae & 6 & 36 & 2 & 14 & 13 & 9 \\
\hline Lyco/Monilophyta & Selaginellaceae & 1 & 23 & & 2 & 16 & 5 \\
\hline Angiosp.-Dicot. & Simaroubaceae & 5 & 7 & & 1 & 5 & 1 \\
\hline Angiosp.-Dicot. & Siparunaceae & 1 & 2 & & & 2 & \\
\hline Angiosp.-Monocot. & Smilacaceae & 1 & 14 & & 2 & 12 & \\
\hline Angiosp.-Dicot. & Solanaceae & 40 & 556 & 36 & 283 & 264 & 9 \\
\hline Angiosp.-Dicot. & Sphenocleaceae & 1 & 1 & & & & 1 \\
\hline Angiosp.-Dicot. & Stylidiaceae & 2 & 2 & & 2 & & \\
\hline Angiosp.-Dicot. & Styracaceae & 1 & 9 & & & 9 & \\
\hline Angiosp.-Dicot. & Symplocaceae & 1 & 21 & & 3 & 18 & \\
\hline Angiosp.-Dicot. & Talinaceae & 1 & 5 & & & 3 & 2 \\
\hline Angiosp.-Dicot. & Tamaricaceae & 1 & 1 & & & & 1 \\
\hline Angiosp.-Monocot. & Tecophilaeaceae & 3 & 9 & 1 & 8 & 1 & \\
\hline Lyco/Monilophyta & Tectariaceae & 1 & 3 & & & 3 & \\
\hline
\end{tabular}


Tabla 3. (Continuación).

\begin{tabular}{|c|c|c|c|c|c|c|c|}
\hline Grupo & Familia & Géneros & Especies & Infraesp. & Endémicas & Nativas & Introd. \\
\hline Angiosp.-Dicot. & Tetrachondraceae & 1 & 1 & & 1 & & \\
\hline Angiosp.-Dicot. & Theaceae & 2 & 2 & & & 1 & 1 \\
\hline Lyco/Monilophyta & Thelypteridaceae & 8 & 62 & 3 & 10 & 48 & 4 \\
\hline Angiosp.-Dicot. & Thesiaceae & 1 & 2 & & & 2 & \\
\hline Angiosp.-Monocot. & Thismiaceae & 1 & 1 & & 1 & & \\
\hline Angiosp.-Dicot. & Thymelaeaceae & 3 & 9 & & 4 & 5 & \\
\hline Angiosp.-Dicot. & Trigoniaceae & 1 & 2 & 1 & & 2 & \\
\hline Angiosp.-Monocot. & Triuridaceae & 2 & 2 & & & 2 & \\
\hline Angiosp.-Dicot. & Tropaeolaceae & 1 & 34 & 5 & 28 & 5 & 1 \\
\hline Angiosp.-Dicot. & Turneraceae & 3 & 33 & 6 & 7 & 26 & \\
\hline Angiosp.-Monocot. & Typhaceae & 1 & 4 & & & 4 & \\
\hline Angiosp.-Dicot. & Ulmaceae & 2 & 2 & & & 1 & 1 \\
\hline Angiosp.-Dicot. & Urticaceae & 13 & 51 & 2 & 13 & 32 & 6 \\
\hline Angiosp.-Monocot. & Velloziaceae & 2 & 4 & & 1 & 3 & \\
\hline Angiosp.-Dicot. & Verbenaceae & 25 & 247 & 28 & 129 & 117 & 1 \\
\hline Angiosp.-Dicot. & Viburnaceae & 2 & 4 & & & 3 & 1 \\
\hline Angiosp.-Dicot. & Violaceae & 6 & 133 & 14 & 104 & 25 & 4 \\
\hline Angiosp.-Dicot. & Viscaceae & 1 & 23 & & 1 & 22 & \\
\hline Angiosp.-Dicot. & Vitaceae & 3 & 17 & 1 & & 14 & 3 \\
\hline Angiosp.-Dicot. & Vochysiaceae & 3 & 12 & 1 & 1 & 11 & \\
\hline Angiosp.-Dicot. & Winteraceae & 1 & 5 & 2 & 4 & 1 & \\
\hline Lyco/Monilophyta & Woodsiaceae & 1 & 1 & & & 1 & \\
\hline Angiosp.-Monocot. & Xyridaceae & 2 & 32 & & 3 & 29 & \\
\hline Angiosp.-Monocot. & Zingiberaceae & 2 & 2 & & & 1 & 1 \\
\hline Angiosp.-Monocot. & Zosteraceae & 1 & 1 & & 1 & & \\
\hline \multirow[t]{2}{*}{ Angiosp.-Dicot. } & Zygophyllaceae & 10 & 20 & & 10 & 9 & 1 \\
\hline & 289 & 2813 & 18931 & 1713 & 7713 & 9892 & 1326 \\
\hline
\end{tabular}

De las 289 familias presentes en el Cono Sur, las diez mejor representadas son Asteraceae, con 2593 especies (1404 endémicas), Poaceae 1563 (544), Fabaceae 1437 (632), Orchidaceae 983 (251), Solanaceae 556 (283), Malvaceae 533 (202), Cyperaceae 516 (163), Euphorbiaceae 453 (188), Cactaceae 436 (311) y Apocynaceae 348 (129). Estas 10 familias incluyen ca. 50\% del total de especies citadas para el Cono Sur. Contrariamente, 43 familias (ca. 15\% del total) están representadas en el Cono Sur por sólo 1 especie.
Para ilustrar las modificaciones que se produjeron en el nivel de familia desde 2008 hasta la actualidad, se mencionan los siguientes ejemplos: Agapanthaceae = Amaryllidaceae; Agavaceae = Asparagaceae; Alliaceae = Amaryllidaceae; Anthericaceae $=$ Asparagaceae; Callitrichaceae $=$ Plantaginaceae; Cecropiaceae = Urticaceae; Centrolepidaceae $=$ Restionaceae; Cochlospermaceae $=$ Bixaceae; Desfontainiaceae $=$ Columelliaceae Dipsacaceae $=$ Caprifoliaceae; Donatiaceae = Stylidaceae; Empetraceae 
= Ericaceae; Epacridaceae = Ericaceae; Eucryphiaceae $=$ Cunoniaceae $;$ Fumariaceae $=$ Papaveraceae; Gilliesiaceae = Amaryllidaceae; Herreriaceae = Asparagaceae; Hippuridaceae = Plantaginaceae; Hyacinthaceae = Asparagaceae; Hydnoraceae $=$ Aristolochiaceae; Laxmanniaceae $=$ Asparagaceae; Ledocarpaceae = Francoaceae; Lemnaceae = Araceae; Limnocharitaceae $=$ Alismataceae Luzuriagaceae = Alstroemeriaceae; Myoporaceae = Scrophulariaceae $;$ Myrsinaceae $=$ Primulaceae; Najadaceae $=$ Hydrocharitaceae Parnassiaceae = Celastraceae; Ruscaceae = Asparagaceae; Samolaceae $=$ Primulaceae; Sterculiaceae $=$ Malvaceae; Tribeleaceae = Escalloniaceae; Valerianaceae = Caprifoliaceae; Vivianiaceae = Francoaceae; Ximeniaceae = Olacaceae; Zannicheliaceae $=$ Potamogetonaceae.

A nivel de género los cambios evidenciados devienen de la descripción de nuevos géneros, algunos a partir de especies segregadas de otros, originalmente mayores en número, entre éstos pueden mencionarse: Aimara, Alshehbazia y Atacama (Brassicaceae), segregados de Menonvillea, Eudema y Sisymbrium, respectivamente; Arquita y Libidibia (Fabaceae), segregados de Caesalpinia; Austroeupatorium, Barrosoa, etc. (Asteraceae) segregados de Eupatorium; Brargentina (Apocynaceae), segregado de Gyrostelma; Famatinanthus (Asteraceae), segregado de Aphyllocladus; Hildaea y Stapfochloa (Poaceae), segregados de Ichnanthus y Chloris, respectivamente; Oedochloa, Osvaldoa, Rugoloa y Trichanthecium (Poaceae), segregados de Panicum; Rhodoscirpus (Cyperaceae), segregado de Scirpus; Spegazziniophyton (Euphorbiaceae), segregado de Colliguaja; Camonea y Distimake (Convolvulaceae) segregados de Convolvulus; Grausa (Loasaceae), segregado de Loasa; Austroblechnum, Cranfilia, Lomaridium y Parablechnum (Blechnaceae), segregados de Blechnum. Entre los géneros fundados recientemente, algunos endémicos para el Cono Sur, se pueden indicar: Topea y Tressenia (Apocynaceae), Kieslingia (Asteraceae) y Zuloagocardamum (Brassicaceae). Contrariamente, algunos géneros han sido incluidos en sinonimia de otros, entre ellos podemos citar a modo de ejemplo: Huanaca $=$ Laretia $=$ Mulinum $=$ Azorella (Apiaceae); Pennisetum $=$ Cenchrus (Poaceae); Spartina $=$ Sporobolus (Poaceae).

Los diez géneros más numerosos en el Cono Sur son Senecio (Asteraceae, 415 especies), Solanum (Solanaceae, 216), Baccharis (Asteraceae, 210), Adesmia (Fabaceae, 200), Mimosa (Fabaceae, 177), Croton (Euphorbiaceae, 134), Carex (Cyperaceae, 133), Miconia (Melastomataceae, 123), Oxalis (Oxalidaceae, 122) y Paspalum (Poaceae, 115). En el extremo opuesto, 1141 géneros (el 40,6\% del total de géneros del Cono Sur) están representados por sólo 1 especie, 466 géneros (el 16,6\%) por 2 especies y 224 géneros (el 8\%) por 3 especies (Tabla 4).

Tabla 4. Géneros presentes en el Cono Sur con detalle del número de especies con el que están representados en el área. Se resaltan los géneros no citados para el Cono Sur en 2008: con $\left(^{*}\right)$ se indican los géneros fundados recientemente, con $(* *)$ aquellos anteriores pero que no habían sido citados previamente.

\begin{tabular}{|l|l|c|}
\hline Género & Familia & $\mathbf{N}^{\mathbf{0}}$ Sp. \\
\hline Aa & Orchidaceae & 4 \\
\hline Abarema & Fabaceae & 2 \\
\hline Abatia & Salicaceae & 2 \\
\hline Abildgaardia & Cyperaceae & 1 \\
\hline Abobra & Cucurbitaceae & 1 \\
\hline Abolboda $\left(^{* *}\right)$ & Xyridaceae & 1 \\
\hline Abrodictyum $(* *)$ & Hymenophyllaceae & 1 \\
\hline Abrotanella & Asteraceae & 7 \\
\hline
\end{tabular}

\begin{tabular}{|l|l|c|}
\hline Género & Familia & $\mathbf{N}^{\mathbf{0}}$ Sp. \\
\hline Abrus $\left(^{* *}\right)$ & Fabaceae & 1 \\
\hline Abuta & Menispermaceae & 1 \\
\hline Abutilon & Malvaceae & 11 \\
\hline Acaciella $\left(^{* *}\right)$ & Fabaceae & 1 \\
\hline Acaena & Rosaceae & 25 \\
\hline Acalypha & Euphorbiaceae & 24 \\
\hline Acanthocalycium & Cactaceae & 5 \\
\hline Acanthocladus & Polygalaceae & 2 \\
\hline
\end{tabular}


Tabla 4. (Continuación).

\begin{tabular}{|c|c|c|}
\hline Género & Familia & $\mathbf{N}^{0}$ Sp. \\
\hline Acantholippia & Verbenaceae & 1 \\
\hline Acanthospermum & Asteraceae & 3 \\
\hline Acanthostachys & Bromeliaceae & 1 \\
\hline Acanthostyles (**) & Asteraceae & 1 \\
\hline Acanthosyris & Cervantesiaceae & 2 \\
\hline Acaulimalva & Malvaceae & 1 \\
\hline$A c c a$ & Myrtaceae & 1 \\
\hline Acer & Aceraceae & 2 \\
\hline Achatocarpus & Achatocarpaceae & 5 \\
\hline Achetaria & Plantaginaceae & 1 \\
\hline Achillea & Asteraceae & 2 \\
\hline Achyrocline & Asteraceae & 19 \\
\hline Aciachne & Poaceae & 1 \\
\hline Acianthera & Orchidaceae & 62 \\
\hline Acicarpha & Calyceraceae & 5 \\
\hline Acilepidopsis (**) & Asteraceae & 1 \\
\hline Aciotis & Melastomataceae & 1 \\
\hline Acisanthera & Melastomataceae & 5 \\
\hline Acmella & Asteraceae & 13 \\
\hline Acnistus & Solanaceae & 1 \\
\hline Acosmium & Fabaceae & 2 \\
\hline Acrisione (**) & Asteraceae & 2 \\
\hline Acroceras & Poaceae & 2 \\
\hline Acrocomia & Arecaceae & 3 \\
\hline Acronia & Orchidaceae & 1 \\
\hline Acrostichum & Pteridaceae & 2 \\
\hline Actinocephalus & Eriocaulaceae & 1 \\
\hline Actinoseris & Asteraceae & 1 \\
\hline Actinostachys & Schizaeaceae & 2 \\
\hline Actinostemon & Euphorbiaceae & 4 \\
\hline Adelia & Euphorbiaceae & 1 \\
\hline Adenaria & Lythraceae & 1 \\
\hline Adenocalymma & Bignoniaceae & 9 \\
\hline Adenocarpus & Fabaceae & 1 \\
\hline Adenocaulon & Asteraceae & 1 \\
\hline Adenopeltis & Euphorbiaceae & 1 \\
\hline Adenostemma & Asteraceae & 3 \\
\hline Adesmia & Fabaceae & 200 \\
\hline Adetogramma (*) & Polypodiaceae & 1 \\
\hline
\end{tabular}

\begin{tabular}{|c|c|c|}
\hline Género & Familia & $\mathbf{N}^{0}$ Sp. \\
\hline Adiantopsis & Pteridaceae & 11 \\
\hline Adiantum & Pteridaceae & 37 \\
\hline Aechmea & Bromeliaceae & 27 \\
\hline Aegiphila & Lamiaceae & 12 \\
\hline Aeschynomene & Fabaceae & 22 \\
\hline Aextoxicon & Aextoxicaceae & 1 \\
\hline Agalinis & Orobanchaceae & 7 \\
\hline Agapanthus & Amaryllidaceae & 1 \\
\hline Agarista & Ericaceae & 9 \\
\hline Agave & Asparagaceae & 1 \\
\hline Agenium & Poaceae & 3 \\
\hline Ageratina (**) & Asteraceae & 4 \\
\hline Ageratum & Asteraceae & 2 \\
\hline Agonandra & Opiliaceae & 2 \\
\hline Agoseris & Asteraceae & 2 \\
\hline Agrimonia & Rosaceae & 3 \\
\hline Agropyron & Poaceae & 1 \\
\hline Agrostemma & Caryophyllaceae & 1 \\
\hline Agrostis & Poaceae & 32 \\
\hline Ailanthus & Simaroubaceae & 1 \\
\hline Aimara (*) & Brassicaceae & 1 \\
\hline Aiouea & Lauraceae & 8 \\
\hline Aira & Poaceae & 4 \\
\hline Airampoa (**) & Cactaceae & 1 \\
\hline Alansmia (*) & Polypodiaceae & 1 \\
\hline Albizia & Fabaceae & 8 \\
\hline Alcea & Malvaceae & 1 \\
\hline Alchornea & Euphorbiaceae & 4 \\
\hline Aldama (**) & Asteraceae & 30 \\
\hline Aleurites $(* *)$ & Euphorbiaceae & 1 \\
\hline Alibertia & Rubiaceae & 2 \\
\hline Alicia & Malpighiaceae & 1 \\
\hline Alisma & Alismataceae & 2 \\
\hline Allagoptera & Arecaceae & 3 \\
\hline Allamanda & Apocynaceae & 2 \\
\hline Allenrolfea & Chenopodiaceae & 2 \\
\hline Alliaria & Brassicaceae & 1 \\
\hline Allionia & Nyctaginaceae & 2 \\
\hline Allium & Amaryllidaceae & 7 \\
\hline
\end{tabular}


Tabla 4. (Continuación).

\begin{tabular}{|c|c|c|}
\hline Género & Familia & $\mathbf{N}^{0}$ Sp. \\
\hline Allophylus & Sapindaceae & 6 \\
\hline Almeidea (**) & Rutaceae & 1 \\
\hline Alnus & Betulaceae & 4 \\
\hline $\operatorname{Alocasia}(* *)$ & Araceae & 1 \\
\hline Alonsoa & Scrophulariaceae & 2 \\
\hline Alopecurus & Poaceae & 9 \\
\hline Aloysia & Verbenaceae & 25 \\
\hline Alseis & Rubiaceae & 1 \\
\hline Alshehbazia (*) & Brassicaceae & 3 \\
\hline Alsophila & Cyatheaceae & 6 \\
\hline Alstroemeria & Alstroemeriaceae & 51 \\
\hline Alternanthera & Amaranthaceae & 36 \\
\hline Alvaradoa & Picramniaceae & 1 \\
\hline Alysicarpus (**) & Fabaceae & 2 \\
\hline Alyssum & Brassicaceae & 1 \\
\hline Amaioua & Rubiaceae & 2 \\
\hline Amaranthus & Amaranthaceae & 30 \\
\hline Amaryllis & Amaryllidaceae & 1 \\
\hline Amasonia & Lamiaceae & 1 \\
\hline Amauropelta $(* *)$ & Thelypteridaceae & 31 \\
\hline Amblyopappus & Asteraceae & 1 \\
\hline Ambrosia & Asteraceae & 8 \\
\hline Amburana & Fabaceae & 1 \\
\hline Ameghinoa & Asteraceae & 1 \\
\hline Amelichloa & Poaceae & 4 \\
\hline Amicia & Fabaceae & 3 \\
\hline Ammannia & Lythraceae & 4 \\
\hline Ammi & Apiaceae & 2 \\
\hline Ammophila & Poaceae & 1 \\
\hline Ammoselinum & Apiaceae & 1 \\
\hline Amomyrtella & Myrtaceae & 1 \\
\hline Amomyrtus & Myrtaceae & 2 \\
\hline Amorimia & Malpighiaceae & 1 \\
\hline Amorpha & Fabaceae & 1 \\
\hline Amphibromus & Poaceae & 1 \\
\hline Amphilophium & Bignoniaceae & 11 \\
\hline Amphipetalum & Portulacaceae & 1 \\
\hline Amphiscirpus & Cyperaceae & 1 \\
\hline Amsinckia & Boraginaceae & 2 \\
\hline
\end{tabular}

\begin{tabular}{|c|c|c|}
\hline Género & Familia & $\mathbf{N}^{0}$ Sp. \\
\hline Anacampseros & Anacampserotaceae & 2 \\
\hline Anacardium & Anacardiaceae & 1 \\
\hline Anadenanthera & Fabaceae & 2 \\
\hline Anagallis & Primulaceae & 3 \\
\hline Ananas & Bromeliaceae & 3 \\
\hline Anaphalis & Asteraceae & 1 \\
\hline Anarthrophyllum & Fabaceae & 15 \\
\hline Anathallis & Orchidaceae & 37 \\
\hline Anatherostipa & Poaceae & 8 \\
\hline Anchietea & Violaceae & 2 \\
\hline Anchusa & Boraginaceae & 1 \\
\hline Ancistrotropis (*) & Fabaceae & 4 \\
\hline Andeimalva & Malvaceae & 1 \\
\hline Andira & Fabaceae & 4 \\
\hline Andropogon & Poaceae & 17 \\
\hline Androsace & Primulaceae & 1 \\
\hline Androtrichum & Cyperaceae & 2 \\
\hline Anemia & Anemiaceae & 15 \\
\hline Anemonastrum (**) & Ranunculaceae & 1 \\
\hline Anemone & Ranunculaceae & 6 \\
\hline Anemopaegma & Bignoniaceae & 7 \\
\hline Anethum & Apiaceae & 1 \\
\hline Angelonia & Plantaginaceae & 2 \\
\hline Aniba & Lauraceae & 1 \\
\hline Aniseia & Convolvulaceae & 3 \\
\hline Anisocapparis (*) & Capparaceae & 1 \\
\hline Anisomeria & Phytolaccaceae & 2 \\
\hline Annona & Annonaceae & 21 \\
\hline Anoda & Malvaceae & 2 \\
\hline Anogramma & Pteridaceae & 1 \\
\hline Anomospermum (**) & Menispermaceae & 1 \\
\hline Anredera & Basellaceae & 4 \\
\hline Antennaria & Asteraceae & 2 \\
\hline Anthaenantia & Poaceae & 1 \\
\hline Anthaenantiopsis & Poaceae & 4 \\
\hline Anthemis & Asteraceae & 2 \\
\hline Anthericum & Asparagaceae & 4 \\
\hline Anthodon & Celastraceae & 1 \\
\hline Anthoxanthum & Poaceae & 7 \\
\hline
\end{tabular}


Tabla 4. (Continuación).

\begin{tabular}{|c|c|c|c|c|c|}
\hline Género & Familia & $\mathbf{N}^{\circ} \mathrm{Sp}$ & Género & Familia & $\mathbf{N}^{0}$ Sp. \\
\hline Anthriscus & Apiaceae & 2 & Argylia & Bignoniaceae & 13 \\
\hline Anthurium & Araceae & 16 & Argyranthemum & Asteraceae & 1 \\
\hline Antidaphne & Eremolepidaceae & 1 & Argyrochosma & Pteridaceae & 4 \\
\hline Antigonon & Polygonaceae & 1 & Aristeguietia (**) & Asteraceae & 1 \\
\hline Antiphytum & Boraginaceae & 1 & Aristida & Poaceae & 45 \\
\hline Antirrhinum & Plantaginaceae & 1 & Aristolochia & Aristolochiaceae & 37 \\
\hline Apalanthe & Hydrocharitaceae & 1 & Aristotelia & Elaeocarpaceae & 1 \\
\hline Aparisthmium & Euphorbiaceae & 1 & Arjona & Schoepfiaceae & 5 \\
\hline Apera & Poaceae & 1 & Armeria & Plumbaginaceae & 1 \\
\hline Aphanes & Rosaceae & 5 & Arnica & Asteraceae & 1 \\
\hline Aphelandra & Acanthaceae & 7 & Arquita (*) & Fabaceae & 2 \\
\hline Aphyllocladus & Asteraceae & 4 & Arrhenatherum & Poaceae & 1 \\
\hline Apinagia & Podostemaceae & 4 & Artemisia & Asteraceae & 9 \\
\hline Apium & Apiaceae & 9 & Arthropogon & Poaceae & 2 \\
\hline Apoclada & Poaceae & 1 & Arthropteris & Oleandraceae & 1 \\
\hline Apodanthera & Cucurbitaceae & 6 & Arthrosia $(* *)$ & Orchidaceae & 5 \\
\hline Apodasmia & Restionaceae & 1 & Artocarpus & Moraceae & 1 \\
\hline Aponogeton & Aponogetonaceae & 1 & Arum & Araceae & 1 \\
\hline Apopyros & Asteraceae & 1 & Arundinella & Poaceae & 2 \\
\hline Aptenia & Aizoaceae & 1 & Arundo & Poaceae & 1 \\
\hline Apteria & Burmanniaceae & 1 & Aschersoniodoxa & Brassicaceae & 1 \\
\hline Apuleia & Fabaceae & 1 & Asclepias & Apocynaceae & 11 \\
\hline Apurimacia & Fabaceae & 1 & Asemeia & Polygalaceae & 7 \\
\hline Aquilegia & Ranunculaceae & 1 & Asparagus & Asparagaceae & 6 \\
\hline Arabidopsis & Brassicaceae & 1 & Aspasia & Orchidaceae & 1 \\
\hline Arachis & Fabaceae & 23 & Asperugo & Boraginaceae & 1 \\
\hline Arachniodes & Dryopteridaceae & 1 & Asphodelus & Asphodelaceae & 1 \\
\hline Arachnitis & Corsiaceae & 1 & Aspicarpa & Malpighiaceae & 5 \\
\hline Araeoandra & Francoaceae & 1 & Aspidogyne & Orchidaceae & 14 \\
\hline Aralia & Araliaceae & 2 & Aspidosperma & Anacardiaceae & 2 \\
\hline Araucaria & Araucariaceae & 2 & Aspilia & Asteraceae & 16 \\
\hline Araujia & Apocynaceae & 11 & Asplenium & Aspleniaceae & 63 \\
\hline Archidasyphyllum (*) & Asteraceae & 2 & Asplundia & Cyclanthaceae & 2 \\
\hline Arctium & Asteraceae & 1 & Astelia & Asteliaceae & 1 \\
\hline Arctotheca & Asteraceae & 1 & Asteranthera & Gesneriaceae & 1 \\
\hline Arctotis & Asteraceae & 1 & Asteriscium & Apiaceae & 9 \\
\hline Ardisia & Primulaceae & 1 & Asteropsis & Asteraceae & 1 \\
\hline Arenaria & Caryophyllaceae & 14 & Asterostigma & Araceae & 2 \\
\hline Argemone & Papaveraceae & 4 & Astraea & Euphorbiaceae & 3 \\
\hline
\end{tabular}


Tabla 4. (Continuación).

\begin{tabular}{|c|c|c|}
\hline Género & Familia & $\mathbf{N}^{o}$ Sp. \\
\hline Astragalus & Fabaceae & 97 \\
\hline Astrocaryum & Arecaceae & 1 \\
\hline Astrolepis & Pteridaceae & 1 \\
\hline Astronium & Anacardiaceae & 3 \\
\hline $\operatorname{Atacama}(*)$ & Brassicaceae & 1 \\
\hline Atamisquea (**) & Capparaceae & 1 \\
\hline Ateleia & Fabaceae & 1 \\
\hline Athroostachys (**) & Poaceae & 1 \\
\hline Athyana & Sapindaceae & 1 \\
\hline Athyrium & Athyriaceae & 1 \\
\hline Atriplex & Chenopodiaceae & 50 \\
\hline Attalea & Arecaceae & 3 \\
\hline Augustea $(* *)$ & Caryophyllaceae & 3 \\
\hline Aulonemia & Poaceae & 5 \\
\hline Aureliana & Solanaceae & 5 \\
\hline Austroblechnum (*) & Blechnaceae & 11 \\
\hline Austrobrickellia (**) & Asteraceae & 2 \\
\hline Austrocactus & Cactaceae & 5 \\
\hline Austrocedrus & Cupressaceae & 1 \\
\hline Austrochthamalia (*) & Apocynaceae & 2 \\
\hline Austrocritonia $(* *)$ & Asteraceae & 1 \\
\hline Austrocylindropuntia & Cactaceae & 4 \\
\hline Austroeupatorium (**) & Asteraceae & 7 \\
\hline Austrolycopodium (**) & Lycopodiaceae & 5 \\
\hline Austropeucedanum & Apiaceae & 1 \\
\hline Austrostipa & Poaceae & 2 \\
\hline Avellanita & Euphorbiaceae & 1 \\
\hline Avena & Poaceae & 6 \\
\hline Avenella (**) & Poaceae & 1 \\
\hline Averrhoidium & Sapindaceae & 1 \\
\hline Avicennia & Acanthaceae & 2 \\
\hline Axonopus & Poaceae & 22 \\
\hline Ayapana (**) & Asteraceae & 1 \\
\hline Ayapanopsis (**) & Asteraceae & 2 \\
\hline Ayenia & Malvaceae & 14 \\
\hline Aylacophora & Asteraceae & 1 \\
\hline Azara & Salicaceae & 10 \\
\hline Azolla & Salviniaceae & 2 \\
\hline Azorella & Apiaceae & 34 \\
\hline
\end{tabular}

\begin{tabular}{|c|c|c|}
\hline Género & Familia & $\mathbf{N}^{0}$ Sp. \\
\hline Baccharis & Asteraceae & 210 \\
\hline Bacopa & Plantaginaceae & 16 \\
\hline Bactris & Arecaceae & 5 \\
\hline Bahia & Asteraceae & 1 \\
\hline Bakerolimon & Plumbaginaceae & 1 \\
\hline Balbisia & Francoaceae & 9 \\
\hline Balfourodendron & Rutaceae & 1 \\
\hline Ballota & Lamiaceae & 1 \\
\hline Balsamocarpon & Fabaceae & 1 \\
\hline Baltimora & Asteraceae & 1 \\
\hline Banara & Salicaceae & 4 \\
\hline Banisteriopsis & Malpighiaceae & 17 \\
\hline Barbacenia & Velloziaceae & 1 \\
\hline Barbaceniopsis & Velloziaceae & 3 \\
\hline Barbarea & Brassicaceae & 2 \\
\hline Barbosella & Orchidaceae & 7 \\
\hline Barjonia & Apocynaceae & 1 \\
\hline Barleria (**) & Acanthaceae & 1 \\
\hline Barnadesia & Asteraceae & 1 \\
\hline Barneoudia & Ranunculaceae & 3 \\
\hline Barrosoa (**) & Asteraceae & 4 \\
\hline Basistemon & Plantaginaceae & 1 \\
\hline Baskervilla & Orchidaceae & 1 \\
\hline Bassia & Chenopodiaceae & 2 \\
\hline Bastardia & Malvaceae & 2 \\
\hline Bastardiopsis & Malvaceae & 1 \\
\hline Bathysa & Rubiaceae & 1 \\
\hline Bauhinia & Fabaceae & 16 \\
\hline Beauverdia (**) & Amaryllidaceae & 4 \\
\hline Becquerelia & Cyperaceae & 2 \\
\hline Begonia & Begoniaceae & 61 \\
\hline Behuria & Melastomataceae & 1 \\
\hline Beilschmiedia & Lauraceae & 2 \\
\hline Bejaranoa (**) & Asteraceae & 1 \\
\hline Belamcanda & Iridaceae & 1 \\
\hline Bellardia (**) & Orobanchaceae & 2 \\
\hline Bellis & Asteraceae & 1 \\
\hline Belloa & Asteraceae & 2 \\
\hline Beloglottis & Orchidaceae & 1 \\
\hline
\end{tabular}


Tabla 4. (Continuación).

\begin{tabular}{|c|c|c|}
\hline Género & Familia & $\mathbf{N}^{0}$ Sp. \\
\hline Benthamiella & Solanaceae & 12 \\
\hline Berberidopsis & Berberidopsidaceae & 1 \\
\hline Berberis & Berberidaceae & 36 \\
\hline Bernardia & Euphorbiaceae & 15 \\
\hline Berroa & Asteraceae & 1 \\
\hline Bertolonia & Melastomataceae & 4 \\
\hline Beschorneria (**) & Asparagaceae & 1 \\
\hline Besleria & Gesneriaceae & 1 \\
\hline Beta & Chenopodiaceae & 1 \\
\hline Betula & Betulaceae & 1 \\
\hline $\operatorname{Bia}(* *)$ & Euphorbiaceae & 1 \\
\hline Bidens & Asteraceae & 18 \\
\hline Bifrenaria & Orchidaceae & 6 \\
\hline Bignonia & Bignoniaceae & 4 \\
\hline Billbergia & Bromeliaceae & 9 \\
\hline Bipinnula & Orchidaceae & 10 \\
\hline Bishovia (**) & Asteraceae & 1 \\
\hline Bixa & Bixaceae & 1 \\
\hline Blackstonia & Gentianaceae & 1 \\
\hline Blandowia & Podostemaceae & 1 \\
\hline Blechnum & Blechnaceae & 18 \\
\hline Blennosperma & Asteraceae & 1 \\
\hline Blepharidachne & Poaceae & 2 \\
\hline Blepharocalyx & Myrtaceae & 2 \\
\hline Blepharodon & Apocynaceae & 6 \\
\hline Bletia & Orchidaceae & 2 \\
\hline Blitum (**) & Chenopodiaceae & 1 \\
\hline Blossfeldia & Cactaceae & 1 \\
\hline Blotiella (**) & Dennstaedtiaceae & 1 \\
\hline Blumenbachia & Loasaceae & 10 \\
\hline Blutaparon & Amaranthaceae & 1 \\
\hline Bocconia & Papaveraceae & 1 \\
\hline Boehmeria & Urticaceae & 6 \\
\hline Boerhavia & Nyctaginaceae & 5 \\
\hline Bolax & Apiaceae & 2 \\
\hline Bolbitis & Dryopteridaceae & 2 \\
\hline Bolboschoenus & Cyperaceae & 2 \\
\hline Bomarea & Alstroemeriaceae & 8 \\
\hline Bonamia & Convolvulaceae & 4 \\
\hline
\end{tabular}

\begin{tabular}{|c|c|c|}
\hline Género & Familia & $\mathbf{N}^{0}$ Sp. \\
\hline Boopis & Calyceraceae & 10 \\
\hline Boquila & Lardizabalaceae & 1 \\
\hline Borago & Boraginaceae & 1 \\
\hline Bordasia & Malvaceae & 1 \\
\hline Borreria & Rubiaceae & 26 \\
\hline Bothriochloa & Poaceae & 17 \\
\hline Botrychium & Ophioglossaceae & 1 \\
\hline Botrypus $(* *)$ & Ophioglossaceae & 1 \\
\hline Bouchea & Verbenaceae & 2 \\
\hline Bouchetia & Solanaceae & 1 \\
\hline Bougainvillea & Nyctaginaceae & 7 \\
\hline Bouteloua & Poaceae & 6 \\
\hline Bowdichia & Fabaceae & 1 \\
\hline Bowlesia & Apiaceae & 12 \\
\hline Brachionidium & Orchidaceae & 1 \\
\hline Brachyclados & Asteraceae & 3 \\
\hline Brachylaena (**) & Asteraceae & 1 \\
\hline Brachyotum & Melastomataceae & 1 \\
\hline Brachypodium & Poaceae & 2 \\
\hline Brachystele & Orchidaceae & 15 \\
\hline Brargentina (*) & Apocynaceae & 1 \\
\hline Brasiliopuntia & Cactaceae & 2 \\
\hline Brasiliorchis (*) & Orchidaceae & 2 \\
\hline Brassavola & Orchidaceae & 2 \\
\hline Brassica & Brassicaceae & 6 \\
\hline Brayopsis & Brassicaceae & 2 \\
\hline Bredemeyera & Polygalaceae & 5 \\
\hline Breynia & Euphorbiaceae & 1 \\
\hline Brickellia & Asteraceae & 1 \\
\hline Bridgesia & Sapindaceae & 1 \\
\hline Briquetia & Malvaceae & 1 \\
\hline Briza & Poaceae & 3 \\
\hline Bromelia & Bromeliaceae & 4 \\
\hline Bromidium & Poaceae & 5 \\
\hline Bromus & Poaceae & 33 \\
\hline Brosimum & Moraceae & 3 \\
\hline Broussonetia & Moraceae & 1 \\
\hline Browningia & Cactaceae & 2 \\
\hline Brugmansia & Solanaceae & 1 \\
\hline
\end{tabular}


Tabla 4. (Continuación).

\begin{tabular}{|c|c|c|}
\hline Género & Familia & $\mathbf{N}^{0} \mathrm{Sp}$. \\
\hline Brunfelsia & Solanaceae & 7 \\
\hline Brunsvigia & Amaryllidaceae & 1 \\
\hline Bryantiella & Polemoniaceae & 1 \\
\hline Bryophyllum (**) & Crassulaceae & 4 \\
\hline Buchenavia & Combretaceae & 1 \\
\hline Buchnera & Orobanchaceae & 6 \\
\hline Buchtienia & Orchidaceae & 1 \\
\hline Buddleja & Scrophulariaceae & 26 \\
\hline Buglossoides (**) & Boraginaceae & 1 \\
\hline Bulbophyllum & Orchidaceae & 25 \\
\hline Bulbostylis & Cyperaceae & 35 \\
\hline Bulnesia & Zygophyllaceae & 4 \\
\hline Bunchosia & Malpighiaceae & 3 \\
\hline Bupleurum & Apiaceae & 1 \\
\hline Burkartia & Asteraceae & 1 \\
\hline Burmannia & Burmanniaceae & 4 \\
\hline Butia & Arecaceae & 17 \\
\hline Byrsonima & Malpighiaceae & 12 \\
\hline Byttneria & Malvaceae & 22 \\
\hline Caamembeca (*) & Polygalaceae & 3 \\
\hline Cabomba & Cabombaceae & 3 \\
\hline Cabralea & Meliaceae & 1 \\
\hline Cabreraea (*) & Asteraceae & 1 \\
\hline Caesalpinia & Fabaceae & 2 \\
\hline Caesarea & Francoaceae & 1 \\
\hline Caiophora & Loasaceae & 19 \\
\hline Cajanus & Fabaceae & 1 \\
\hline Cakile & Brassicaceae & 1 \\
\hline Caladium & Araceae & 1 \\
\hline Calandrinia & Montiaceae & 14 \\
\hline Calceolaria & Calceolariaceae & 81 \\
\hline Caldcluvia & Cunoniaceae & 1 \\
\hline Calea & Asteraceae & 28 \\
\hline Calendula & Asteraceae & 3 \\
\hline Calibrachoa & Solanaceae & 25 \\
\hline Callaeum & Malpighiaceae & 1 \\
\hline Calliandra & Fabaceae & 10 \\
\hline Callianthe (*) & Malvaceae & 13 \\
\hline Callianthemoides & Ranunculaceae & 1 \\
\hline
\end{tabular}

\begin{tabular}{|c|c|c|}
\hline Género & Familia & $\mathbf{N}^{0}$ Sp. \\
\hline Callisia & Commelinaceae & 2 \\
\hline Callisthene & Vochysiaceae & 3 \\
\hline Callitriche & Plantaginaceae & 10 \\
\hline Calolisianthus & Gentianaceae & 4 \\
\hline Calopappus & Asteraceae & 1 \\
\hline Calophyllum & Calophyllaceae & 1 \\
\hline Calopogonium & Fabaceae & 3 \\
\hline Calotropis & Apocynaceae & 1 \\
\hline Caltha & Ranunculaceae & 3 \\
\hline Calycera & Calyceraceae & 9 \\
\hline Calycophyllum & Rubiaceae & 1 \\
\hline Calyculogygas & Malvaceae & 2 \\
\hline Calydorea & Iridaceae & 19 \\
\hline Calyptocarpus & Asteraceae & 2 \\
\hline Calyptraemalva & Malvaceae & 1 \\
\hline Calyptrocarya & Cyperaceae & 1 \\
\hline Calystegia & Convolvulaceae & 4 \\
\hline Camarea & Malpighiaceae & 1 \\
\hline Cambajuva (*) & Poaceae & 1 \\
\hline Cambessedesia & Melastomataceae & 2 \\
\hline Camelina & Brassicaceae & 3 \\
\hline Camellia (**) & Theaceae & 1 \\
\hline Camissonia & Onagraceae & 1 \\
\hline Camonea (**) & Convolvulaceae & 1 \\
\hline Campanula & Campanulaceae & 2 \\
\hline Campomanesia & Myrtaceae & 13 \\
\hline Campovassouria $(* *)$ & Asteraceae & 2 \\
\hline Campsidium & Bignoniaceae & 1 \\
\hline Campsis & Bignoniaceae & 1 \\
\hline Camptosema & Fabaceae & 4 \\
\hline Campuloclinium (**) & Asteraceae & 7 \\
\hline Campylocentrum & Orchidaceae & 21 \\
\hline Campyloneurum & Polypodiaceae & 14 \\
\hline Canastra & Poaceae & 1 \\
\hline Canavalia & Fabaceae & 7 \\
\hline Canna & Cannaceae & 5 \\
\hline Cantinoa (*) & Lamiaceae & 5 \\
\hline Capanemia & Orchidaceae & 7 \\
\hline Caperonia & Euphorbiaceae & 9 \\
\hline
\end{tabular}


Tabla 4. (Continuación).

\begin{tabular}{|c|c|c|}
\hline Género & Familia & $\mathbf{N}^{0}$ Sp. \\
\hline Capparicordis (*) & Capparaceae & 1 \\
\hline Capparidastrum (**) & Capparaceae & 3 \\
\hline Capraria & Scrophulariaceae & 1 \\
\hline Capsella & Brassicaceae & 1 \\
\hline Capsicum & Solanaceae & 6 \\
\hline Cardamine & Brassicaceae & 18 \\
\hline Cardenanthus & Iridaceae & 1 \\
\hline Cardionema & Caryophyllaceae & 5 \\
\hline Cardiospermum & Sapindaceae & 3 \\
\hline Carduus & Asteraceae & 5 \\
\hline Carex & Cyperaceae & 133 \\
\hline Carica & Caricaceae & 2 \\
\hline Cariniana & Lecythidaceae & 2 \\
\hline Carolus & Malpighiaceae & 1 \\
\hline Carpha & Cyperaceae & 1 \\
\hline Carpobrotus & Aizoaceae & 2 \\
\hline Carthamus & Asteraceae & 2 \\
\hline Carya (**) & Juglandaceae & 1 \\
\hline Caryocar & Caryocaraceae & 1 \\
\hline Cascaronia & Fabaceae & 1 \\
\hline Casearia & Salicaceae & 11 \\
\hline Casimirella & Icacinaceae & 1 \\
\hline Casselia & Verbenaceae & 1 \\
\hline Cassia & Fabaceae & 4 \\
\hline Cassytha $(* *)$ & Lauraceae & 1 \\
\hline Castela & Simaroubaceae & 2 \\
\hline Castelnavia & Podostemaceae & 1 \\
\hline Castilleja & Orobanchaceae & 4 \\
\hline Catabrosa & Poaceae & 2 \\
\hline Catapodium & Poaceae & 1 \\
\hline Catasetum & Orchidaceae & 7 \\
\hline Catharanthus & Apocynaceae & 1 \\
\hline Catopsis & Bromeliaceae & 2 \\
\hline Cattleya & Orchidaceae & 10 \\
\hline Caucalis & Apiaceae & 1 \\
\hline Cayaponia & Cucurbitaceae & 20 \\
\hline Cecropia & Urticaceae & 2 \\
\hline Cedrela & Meliaceae & 5 \\
\hline Ceiba & Malvaceae & 4 \\
\hline
\end{tabular}

\begin{tabular}{|c|c|c|}
\hline Género & Familia & $\mathbf{N}^{0}$ Sp. \\
\hline Celastrus (**) & Celastraceae & 1 \\
\hline Celosia & Amaranthaceae & 3 \\
\hline Celtis & Cannabaceae & 5 \\
\hline Cenchropsis (**) & Poaceae & 1 \\
\hline Cenchrus & Poaceae & 17 \\
\hline Cenostigma (**) & Fabaceae & 3 \\
\hline Centaurea & Asteraceae & 18 \\
\hline Centaurium & Gentianaceae & 5 \\
\hline Centaurodendron & Asteraceae & 2 \\
\hline Centella & Apiaceae & 1 \\
\hline Centipeda & Asteraceae & 1 \\
\hline Centranthus (**) & Caprifoliaceae & 1 \\
\hline Centratherum & Asteraceae & 2 \\
\hline Centrolobium & Fabaceae & 2 \\
\hline Centrosema & Fabaceae & 11 \\
\hline Centunculus & Primulaceae & 1 \\
\hline Cephalanthus & Rubiaceae & 1 \\
\hline Ceradenia & Polypodiaceae & 3 \\
\hline Cerastium & Caryophyllaceae & 20 \\
\hline Ceratocephalus & Ranunculaceae & 1 \\
\hline Ceratophyllum & Ceratophyllaceae & 2 \\
\hline Ceratopteris & Pteridaceae & 2 \\
\hline Ceratosanthes & Cucurbitaceae & 3 \\
\hline Ceratostigma $(* *)$ & Plumbaginaceae & 1 \\
\hline Cereus & Cactaceae & 9 \\
\hline Cestrum & Solanaceae & 20 \\
\hline Ceterach & Aspleniaceae & 1 \\
\hline Chacoa $(* *)$ & Asteraceae & 1 \\
\hline Chaerophyllum (**) & Apiaceae & 2 \\
\hline Chaetanthera & Asteraceae & 30 \\
\hline Chaetogastra (**) & Melastomataceae & 6 \\
\hline Chaetostoma & Melastomataceae & 1 \\
\hline Chamaecrista & Fabaceae & 26 \\
\hline Chamissoa & Amaranthaceae & 3 \\
\hline Chaptalia & Asteraceae & 10 \\
\hline Chascolytrum & Poaceae & 21 \\
\hline Chasmanthe & Iridaceae & 1 \\
\hline Cheilanthes & Pteridaceae & 17 \\
\hline Cheiloclinium & Celastraceae & 1 \\
\hline
\end{tabular}


Tabla 4. (Continuación).

\begin{tabular}{|c|c|c|}
\hline Género & Familia & $\mathbf{N}^{0}$ Sp. \\
\hline Cheiroglossa & Ophioglossaceae & 1 \\
\hline Chelidonium & Papaveraceae & 1 \\
\hline Chelonanthus & Gentianaceae & 3 \\
\hline Chenopodiastrum (*) & Chenopodiaceae & 1 \\
\hline Chenopodium & Chenopodiaceae & 26 \\
\hline Chersodoma & Asteraceae & 5 \\
\hline Chevreulia & Asteraceae & 6 \\
\hline Chiliophyllum & Asteraceae & 1 \\
\hline Chiliotrichiopsis & Asteraceae & 2 \\
\hline Chiliotrichum & Asteraceae & 2 \\
\hline Chilocardamum & Brassicaceae & 4 \\
\hline Chiococca & Rubiaceae & 1 \\
\hline Chionanthus & Oleaceae & 3 \\
\hline Chionolaena $(* *)$ & Asteraceae & 1 \\
\hline Chiropetalum & Euphorbiaceae & 16 \\
\hline Chlidanthus & Amaryllidaceae & 2 \\
\hline Chloraea & Orchidaceae & 44 \\
\hline Chloris & Poaceae & 12 \\
\hline Chloroleucon & Fabaceae & 3 \\
\hline Chomelia & Rubiaceae & 8 \\
\hline Chondrilla & Asteraceae & 1 \\
\hline Chorispora & Brassicaceae & 1 \\
\hline Chorizanthe & Polygonaceae & 18 \\
\hline Chresta & Asteraceae & 2 \\
\hline Christella (**) & Thelypteridaceae & 3 \\
\hline Christianella & Malpighiaceae & 1 \\
\hline Chromolaena $(* *)$ & Asteraceae & 42 \\
\hline Chrysanthellum & Asteraceae & 1 \\
\hline Chrysanthemoides & Asteraceae & 1 \\
\hline Chrysanthemum & Asteraceae & 2 \\
\hline Chrysolaena (**) & Asteraceae & 17 \\
\hline Chrysophyllum & Sapotaceae & 6 \\
\hline Chrysopogon & Poaceae & 1 \\
\hline Chrysosplenium & Saxifragaceae & 2 \\
\hline Chuquiraga & Asteraceae & 17 \\
\hline Chusquea & Poaceae & 37 \\
\hline Cicendia & Gentianaceae & 1 \\
\hline Cichorium & Asteraceae & 1 \\
\hline Cienfuegosia & Malvaceae & 14 \\
\hline
\end{tabular}

\begin{tabular}{|c|c|c|}
\hline Género & Familia & $\mathbf{N}^{0}$ Sp. \\
\hline Cinnamodendron & Canellaceae & 2 \\
\hline Cinnamoтum & Lauraceae & 4 \\
\hline Cipura & Iridaceae & 1 \\
\hline Cirrhaea & Orchidaceae & 4 \\
\hline Cirsium & Asteraceae & 2 \\
\hline Cissampelos & Menispermaceae & 7 \\
\hline Cissarobryon & Francoaceae & 1 \\
\hline Cissus & Vitaceae & 14 \\
\hline Cistanthe & Montiaceae & 31 \\
\hline Citharexylum & Verbenaceae & 7 \\
\hline Citronella & Cardiopteridaceae & 6 \\
\hline Citrullus & Cucurbitaceae & 2 \\
\hline Citrus & Rutaceae & 2 \\
\hline Cladanthus (**) & Asteraceae & 1 \\
\hline Cladium & Cyperaceae & 2 \\
\hline Clara & Asparagaceae & 3 \\
\hline Clarkia & Onagraceae & 2 \\
\hline Clavija & Primulaceae & 1 \\
\hline Claytonia & Montiaceae & 1 \\
\hline Cleistes & Orchidaceae & 14 \\
\hline Cleistocactus & Cactaceae & 4 \\
\hline Clematis & Ranunculaceae & 6 \\
\hline Cleobulia (**) & Fabaceae & 1 \\
\hline Cleome & Cleomaceae & 3 \\
\hline Cleoserrata (*) & Cleomaceae & 1 \\
\hline Clerodendrum & Lamiaceae & 4 \\
\hline Clethra & Clethraceae & 2 \\
\hline Clibadium & Asteraceae & 2 \\
\hline Clidemia & Melastomataceae & 2 \\
\hline Clinanthus & Amaryllidaceae & 1 \\
\hline Clinopodium & Lamiaceae & 6 \\
\hline Cliococca & Linaceae & 1 \\
\hline Clistax (**) & Acanthaceae & 1 \\
\hline Clitoria & Fabaceae & 9 \\
\hline Clusia & Clusiaceae & 1 \\
\hline Cnicothamnus & Asteraceae & 2 \\
\hline Cnidoscolus & Euphorbiaceae & 13 \\
\hline Cobaea (**) & Polemoniaceae & 1 \\
\hline Coccocypselum & Rubiaceae & 10 \\
\hline
\end{tabular}


Tabla 4. (Continuación).

\begin{tabular}{|c|c|c|}
\hline Género & Familia & $\mathbf{N}^{0}$ Sp. \\
\hline Coccoloba & Polygonaceae & 11 \\
\hline Cochliasanthus (**) & Fabaceae & 1 \\
\hline Cochlidium & Polypodiaceae & 2 \\
\hline Cochlospermum & Bixaceae & 2 \\
\hline Codonanthe & Gesneriaceae & 3 \\
\hline Codonorchis & Orchidaceae & 2 \\
\hline Cohniella (**) & Orchidaceae & 2 \\
\hline Coix & Poaceae & 1 \\
\hline Colanthelia & Poaceae & 3 \\
\hline Coleataenia (**) & Poaceae & 1 \\
\hline Coleostephus & Asteraceae & 1 \\
\hline Colignonia & Nyctaginaceae & 1 \\
\hline Collaea & Fabaceae & 6 \\
\hline Colletia & Rhamnaceae & 6 \\
\hline Colliguaja & Euphorbiaceae & 5 \\
\hline Collomia & Polemoniaceae & 2 \\
\hline Colobanthus & Caryophyllaceae & 3 \\
\hline Colocasia & Araceae & 1 \\
\hline Cologania & Fabaceae & 1 \\
\hline Colubrina & Rhamnaceae & 2 \\
\hline Colutea & Fabaceae & 1 \\
\hline Comanthera (**) & Eriocaulaceae & 1 \\
\hline Combera & Solanaceae & 2 \\
\hline Combretum & Combretaceae & 6 \\
\hline Commelina & Commelinaceae & 9 \\
\hline Commiphora & Burseraceae & 1 \\
\hline Comparettia & Orchidaceae & 1 \\
\hline Conanthera & Tecophilaeaceae & 5 \\
\hline Conchocarpus & Rutaceae & 2 \\
\hline Condalia & Rhamnaceae & 4 \\
\hline Condea (**) & Lamiaceae & 5 \\
\hline Condylocarpon & Apocynaceae & 1 \\
\hline Condylostylis (**) & Fabaceae & 1 \\
\hline Conium & Apiaceae & 1 \\
\hline Connarus & Connaraceae & 2 \\
\hline Conobea (**) & Plantaginaceae & 2 \\
\hline Conocarpus $(* *)$ & Combretaceae & 1 \\
\hline Constantia & Orchidaceae & 2 \\
\hline Convolvulus & Convolvulaceae & 12 \\
\hline
\end{tabular}

\begin{tabular}{|c|c|c|}
\hline Género & Familia & $\mathbf{N}^{0}$ Sp. \\
\hline Conyza & Asteraceae & 43 \\
\hline Copaifera & Fabaceae & 3 \\
\hline Copernicia & Arecaceae & 1 \\
\hline Copiapoa & Cactaceae & 24 \\
\hline Coprosma & Rubiaceae & 2 \\
\hline Corchorus & Malvaceae & 2 \\
\hline Cordia & Boraginaceae & 16 \\
\hline Cordiera & Rubiaceae & 4 \\
\hline Cordobia & Malpighiaceae & 1 \\
\hline Cordyline & Asparagaceae & 2 \\
\hline Coreopsis & Asteraceae & 5 \\
\hline Coriandrum & Apiaceae & 1 \\
\hline Coriaria & Coriariaceae & 1 \\
\hline Coronilla & Fabaceae & 1 \\
\hline Corrigiola & Caryophyllaceae & 3 \\
\hline Corryocactus & Cactaceae & 1 \\
\hline Cortaderia & Poaceae & 6 \\
\hline Corymborkis & Orchidaceae & 1 \\
\hline Corynabutilon & Malvaceae & 7 \\
\hline Corynephorus & Poaceae & 2 \\
\hline Cosmos & Asteraceae & 3 \\
\hline Costus & Costaceae & 3 \\
\hline Cotoneaster & Rosaceae & 4 \\
\hline Cottea & Poaceae & 1 \\
\hline Cotula & Asteraceae & 3 \\
\hline Conepia & Chrysobalanaceae & 2 \\
\hline Coursetia & Fabaceae & 2 \\
\hline Coussapoa & Urticaceae & 1 \\
\hline Coussarea & Rubiaceae & 2 \\
\hline Coutarea & Rubiaceae & 1 \\
\hline Cranfillia (*) & Blechnaceae & 3 \\
\hline Cranichis & Orchidaceae & 5 \\
\hline Craniolaria & Martyniaceae & 1 \\
\hline Crassula & Crassulaceae & 13 \\
\hline Crataegus & Rosaceae & 1 \\
\hline Crateva & Capparaceae & 1 \\
\hline Cratylia & Fabaceae & 1 \\
\hline Cremolobus & Brassicaceae & 2 \\
\hline Crepidomanes (**) & Hymenophyllaceae & 1 \\
\hline
\end{tabular}


Tabla 4. (Continuación).

\begin{tabular}{|c|c|c|}
\hline Género & Familia & $\mathbf{N}^{0}$ Sp. \\
\hline Crepis & Asteraceae & 5 \\
\hline Cressa & Convolvulaceae & 2 \\
\hline Crinodendron & Elaeocarpaceae & 4 \\
\hline Crinum & Amaryllidaceae & 1 \\
\hline Criscia & Asteraceae & 1 \\
\hline Cristaria & Malvaceae & 19 \\
\hline Cristobalia (*) & Apocynaceae & 2 \\
\hline Critonia (**) & Asteraceae & 3 \\
\hline Critoniopsis (**) & Asteraceae & 1 \\
\hline Crocanthemum & Cistaceae & 1 \\
\hline Crocosmia & Iridaceae & 1 \\
\hline Crotalaria & Fabaceae & 26 \\
\hline Croton & Euphorbiaceae & 134 \\
\hline Cruckshanksia & Rubiaceae & 7 \\
\hline Crumenaria & Rhamnaceae & 2 \\
\hline Cryptangium (**) & Cyperaceae & 3 \\
\hline Cryptantha & Boraginaceae & 46 \\
\hline Cryptocarya & Lauraceae & 4 \\
\hline Cryptogramma & Pteridaceae & 1 \\
\hline Ctenanthe & Marantaceae & 10 \\
\hline Ctenitis & Dryopteridaceae & 16 \\
\hline Ctenium & Poaceae & 4 \\
\hline Cuatrecasasiella & Asteraceae & 1 \\
\hline Cucumis & Cucurbitaceae & 3 \\
\hline Cucurbita & Cucurbitaceae & 4 \\
\hline Cucurbitella & Cucurbitaceae & 1 \\
\hline Culcita $(* *)$ & Culcitaceae & 1 \\
\hline Cuminia & Lamiaceae & 1 \\
\hline Cumulopuntia & Cactaceae & 3 \\
\hline Cunila & Lamiaceae & 11 \\
\hline Cupania & Sapindaceae & 3 \\
\hline Cuphea & Lythraceae & 43 \\
\hline Cupressus (**) & Cupressaceae & 2 \\
\hline Curatella & Dilleniaceae & 1 \\
\hline Curculigo (**) & Hypoxidaceae & 1 \\
\hline Curitiba (*) & Myrtaceae & 1 \\
\hline Curtia & Gentianaceae & 2 \\
\hline Cuscuta & Convolvulaceae & 39 \\
\hline Cuspidaria & Bignoniaceae & 4 \\
\hline
\end{tabular}

\begin{tabular}{|c|c|c|}
\hline Género & Familia & $\mathbf{N}^{0}$ Sp. \\
\hline Cyanaeorchis & Orchidaceae & 2 \\
\hline Cyanocephalus (*) & Lamiaceae & 3 \\
\hline Cyathea & Cyatheaceae & 18 \\
\hline Cybianthus & Primulaceae & 2 \\
\hline Cybistax & Bignoniaceae & 1 \\
\hline Cyclanthera & Cucurbitaceae & 8 \\
\hline Cyclodium & Dryopteridaceae & 1 \\
\hline Cyclolepis & Asteraceae & 1 \\
\hline Cyclolobium & Fabaceae & 1 \\
\hline Cycloloma & Chenopodiaceae & 1 \\
\hline Cyclopogon & Orchidaceae & 25 \\
\hline Cyclosorus (*) & Thelypteridaceae & 1 \\
\hline Cyclospermum & Apiaceae & 3 \\
\hline Cylindropuntia & Cactaceae & 2 \\
\hline Cymbalaria & Plantaginaceae & 1 \\
\hline Cymbocarpa & Burmanniaceae & 1 \\
\hline Cymbopogon & Poaceae & 2 \\
\hline Cynanchum & Apocynaceae & 3 \\
\hline Cynara & Asteraceae & 1 \\
\hline Cynodon & Poaceae & 12 \\
\hline Cynoglossum & Boraginaceae & 3 \\
\hline Cynometra & Fabaceae & 1 \\
\hline Cynophalla (**) & Capparaceae & 4 \\
\hline Cynosurus & Poaceae & 2 \\
\hline Cypella & Iridaceae & 34 \\
\hline Cyperus & Cyperaceae & 90 \\
\hline Cyphocarpus & Campanulaceae & 3 \\
\hline Cypselea & Aizoaceae & 1 \\
\hline Cyrtocymura (**) & Asteraceae & 2 \\
\hline Cyrtomium & Dryopteridaceae & 1 \\
\hline Cyrtopodium & Orchidaceae & 17 \\
\hline Cystopteris & Cystopteridaceae & 2 \\
\hline Cytisus & Fabaceae & 2 \\
\hline Dactylaena & Capparaceae & 1 \\
\hline Dactylis & Poaceae & 1 \\
\hline Dactyloctenium & Poaceae & 1 \\
\hline Dahlia & Asteraceae & 1 \\
\hline Dahlstedtia & Fabaceae & 5 \\
\hline Dalbergia & Fabaceae & 8 \\
\hline
\end{tabular}


Tabla 4. (Continuación).

\begin{tabular}{|c|c|c|}
\hline Género & Familia & $\mathbf{N}^{0}$ Sp. \\
\hline Dalea & Fabaceae & 6 \\
\hline Dalechampia & Euphorbiaceae & 22 \\
\hline Danaea & Marattiaceae & 3 \\
\hline Danthonia & Poaceae & 12 \\
\hline Daphnopsis & Thymelaeaceae & 6 \\
\hline Dasyanthina (**) & Asteraceae & 1 \\
\hline Dasycondylus (**) & Asteraceae & 3 \\
\hline Dasyphyllum & Asteraceae & 14 \\
\hline Datura & Solanaceae & 3 \\
\hline Daucus & Apiaceae & 3 \\
\hline Davilla & Dilleniaceae & 3 \\
\hline Declieuxia & Rubiaceae & 4 \\
\hline Deianira (**) & Gentianaceae & 1 \\
\hline Deinacanthon (**) & Bromeliaceae & 1 \\
\hline Delairea $(* *)$ & Asteraceae & 1 \\
\hline Delilia & Asteraceae & 1 \\
\hline Delpinophytum & Brassicaceae & 1 \\
\hline Dendropanax & Araliaceae & 4 \\
\hline Dendrophorbium (**) & Asteraceae & 10 \\
\hline Denisophytum (**) & Fabaceae & 1 \\
\hline Denmoza & Cactaceae & 1 \\
\hline Dennstaedtia & Dennstaedtiaceae & 6 \\
\hline Deparia $(* *)$ & Athyriaceae & 1 \\
\hline Deppea & Rubiaceae & 1 \\
\hline Deschampsia & Poaceae & 20 \\
\hline Descurainia & Brassicaceae & 9 \\
\hline Desfontainia & Columelliaceae & 1 \\
\hline Desmanthus & Fabaceae & 6 \\
\hline Desmaria & Loranthaceae & 1 \\
\hline Desmodium & Fabaceae & 30 \\
\hline Desmoscelis & Melastomataceae & 1 \\
\hline Deuterocohnia & Bromeliaceae & 10 \\
\hline Deyeuxia & Poaceae & 43 \\
\hline Dianthus & Caryophyllaceae & 2 \\
\hline Diastatea (**) & Campanulaceae & 1 \\
\hline Diatenopteryx & Sapindaceae & 1 \\
\hline Dicella & Malpighiaceae & 2 \\
\hline Dichaea & Orchidaceae & 7 \\
\hline Dichanthelium & Poaceae & 6 \\
\hline
\end{tabular}

\begin{tabular}{|c|c|c|}
\hline Género & Familia & $\mathbf{N}^{0}$ Sp. \\
\hline Dichanthium & Poaceae & 2 \\
\hline Dichelachne (**) & Poaceae & 2 \\
\hline Dichondra & Convolvulaceae & 5 \\
\hline Dichorisandra & Commelinaceae & 5 \\
\hline Dicksonia & Dicksoniaceae & 3 \\
\hline Dicliptera & Acanthaceae & 7 \\
\hline Dicranopteris & Gleicheniaceae & 3 \\
\hline Dictyophragmus & Brassicaceae & 1 \\
\hline Dictyostega & Burmanniaceae & 1 \\
\hline Didymochlaena & Didymochlaenaceae & 1 \\
\hline Didymoglossum (**) & Hymenophyllaceae & 7 \\
\hline Dieffenbachia & Araceae & 1 \\
\hline Digitalis & Plantaginaceae & 1 \\
\hline Digitaria & Poaceae & 40 \\
\hline Dilodendron & Sapindaceae & 1 \\
\hline Dimerostemma & Asteraceae & 11 \\
\hline Dimorphandra & Fabaceae & 1 \\
\hline Dinebra $(* *)$ & Poaceae & 1 \\
\hline Dinemagonum & Malpighiaceae & 1 \\
\hline Dinemandra & Malpighiaceae & 1 \\
\hline Dinoseris & Asteraceae & 1 \\
\hline Dioclea & Fabaceae & 4 \\
\hline Diodia & Rubiaceae & 2 \\
\hline Dioscorea & Dioscoreaceae & 101 \\
\hline Diospyros & Ebenaceae & 3 \\
\hline Diostea & Verbenaceae & 1 \\
\hline Diphasiastrum (**) & Lycopodiaceae & 2 \\
\hline Diphasium $(* *)$ & Lycopodiaceae & 2 \\
\hline Diplachne (**) & Poaceae & 1 \\
\hline Diplazium & Athyriaceae & 22 \\
\hline Diplokeleba & Sapindaceae & 1 \\
\hline Diplolepis & Apocynaceae & 14 \\
\hline Diploon & Sapotaceae & 1 \\
\hline Diplopterys & Malpighiaceae & 2 \\
\hline Diplostephium & Asteraceae & 3 \\
\hline Diplotaxis & Brassicaceae & 3 \\
\hline Dipogon & Fabaceae & 1 \\
\hline Diposis & Apiaceae & 3 \\
\hline Dipsacus & Caprifoliaceae & 2 \\
\hline
\end{tabular}


Tabla 4. (Continuación).

\begin{tabular}{|c|c|c|}
\hline Género & Familia & $\mathbf{N}^{0}$ Sp. \\
\hline Dipteryx & Fabaceae & 1 \\
\hline Diptychandra & Fabaceae & 1 \\
\hline Dipyrena & Verbenaceae & 1 \\
\hline Dirhamphis & Malvaceae & 1 \\
\hline Disakisperma (**) & Poaceae & 1 \\
\hline Discaria & Rhamnaceae & 3 \\
\hline Disciphania & Menispermaceae & 2 \\
\hline Discocactus & Cactaceae & 1 \\
\hline Discolobium & Fabaceae & 3 \\
\hline Distichia & Juncaceae & 2 \\
\hline Distichlis & Poaceae & 6 \\
\hline Distimake (**) & Convolvulaceae & 9 \\
\hline Disynaphia (**) & Asteraceae & 11 \\
\hline Ditassa & Apocynaceae & 7 \\
\hline Ditaxis & Euphorbiaceae & 9 \\
\hline Dittrichia & Asteraceae & 1 \\
\hline Dodonaea & Sapindaceae & 1 \\
\hline Dolichandra & Bignoniaceae & 8 \\
\hline Dolichlasium & Asteraceae & 1 \\
\hline Dolichopsis & Fabaceae & 2 \\
\hline Doliocarpus & Dilleniaceae & 4 \\
\hline Domeykoa & Apiaceae & 3 \\
\hline Donatia & Stylidiaceae & 1 \\
\hline Doniophyton & Asteraceae & 2 \\
\hline Dorstenia & Moraceae & 8 \\
\hline Doryopteris & Pteridaceae & 18 \\
\hline Downingia & Campanulaceae & 1 \\
\hline Draba & Brassicaceae & 11 \\
\hline Dracontium & Araceae & 1 \\
\hline Drapetes & Thymelaeaceae & 1 \\
\hline Drimys & Winteraceae & 5 \\
\hline Drosera & Droseraceae & 6 \\
\hline Dryadella & Orchidaceae & 4 \\
\hline Drymaria & Caryophyllaceae & 6 \\
\hline Dryopteris & Dryopteridaceae & 4 \\
\hline Duchesnea & Rosaceae & 1 \\
\hline Duguetia & Annonaceae & 3 \\
\hline Dunalia & Solanaceae & 2 \\
\hline Duranta & Verbenaceae & 3 \\
\hline
\end{tabular}

\begin{tabular}{|c|c|c|}
\hline Género & Familia & $\mathbf{N}^{\circ}$ Sp. \\
\hline Duseniella & Asteraceae & 1 \\
\hline Dyckia & Bromeliaceae & 63 \\
\hline Dyschoriste & Acanthaceae & 11 \\
\hline Dysopsis & Euphorbiaceae & 2 \\
\hline Dysphania & Chenopodiaceae & 12 \\
\hline Dyssochroma & Solanaceae & 2 \\
\hline Dyssodia & Asteraceae & 1 \\
\hline Ecclinusa & Sapotaceae & 1 \\
\hline Eccremocarpus & Bignoniaceae & 1 \\
\hline Echeandia $(* *)$ & Asparagaceae & 1 \\
\hline Echeveria & Crassulaceae & 1 \\
\hline Echinochloa & Poaceae & 7 \\
\hline Echinodorus & Alismataceae & 11 \\
\hline Echinopepon & Cucurbitaceae & 2 \\
\hline Echinopsis & Cactaceae & 9 \\
\hline Echium & Boraginaceae & 2 \\
\hline Eclipta & Asteraceae & 3 \\
\hline Edmundoa & Bromeliaceae & 1 \\
\hline Egeria & Hydrocharitaceae & 2 \\
\hline Egletes & Asteraceae & 1 \\
\hline Ehretia & Boraginaceae & 1 \\
\hline Ehrharta & Poaceae & 3 \\
\hline Eithea & Amaryllidaceae & 1 \\
\hline Elachyptera & Celastraceae & 2 \\
\hline Elaeagnus & Elaeagnaceae & 1 \\
\hline Elaphoglossum & Dryopteridaceae & 57 \\
\hline Elatine & Elatinaceae & 3 \\
\hline Eleocharis & Cyperaceae & 79 \\
\hline Elephantopus & Asteraceae & 3 \\
\hline Eleusine & Poaceae & 2 \\
\hline Eleutheranthera $(* *)$ & Asteraceae & 1 \\
\hline Eleutherine & Iridaceae & 2 \\
\hline Elionurus & Poaceae & 4 \\
\hline Elleanthus & Orchidaceae & 2 \\
\hline Elodea & Hydrocharitaceae & 2 \\
\hline Eltroplectris & Orchidaceae & 4 \\
\hline Elymus & Poaceae & 7 \\
\hline Elytraria & Acanthaceae & 1 \\
\hline Elytrigia (**) & Poaceae & 1 \\
\hline
\end{tabular}


Tabla 4. (Continuación).

\begin{tabular}{|c|c|c|}
\hline Género & Familia & $\mathbf{N}^{0}$ Sp. \\
\hline Elytropus & Apocynaceae & 1 \\
\hline Embothrium & Proteaceae & 1 \\
\hline Emex & Polygonaceae & 1 \\
\hline Emilia & Asteraceae & 2 \\
\hline Emmeorhiza & Rubiaceae & 1 \\
\hline Empetrum & Ericaceae & 1 \\
\hline Encelia & Asteraceae & 1 \\
\hline Encyclia & Orchidaceae & 6 \\
\hline Endlicheria & Lauraceae & 1 \\
\hline Ennealophus & Iridaceae & 4 \\
\hline Enneapogon & Poaceae & 1 \\
\hline Enterolobium & Fabaceae & 2 \\
\hline Enydra & Asteraceae & 2 \\
\hline Ephedra & Ephedraceae & 9 \\
\hline Epidendrum & Orchidaceae & 36 \\
\hline Epilobium & Onagraceae & 17 \\
\hline Epiphyllum & Cactaceae & 1 \\
\hline Epistephium & Orchidaceae & 2 \\
\hline Equisetum & Equisetaceae & 2 \\
\hline Eragrostis & Poaceae & 49 \\
\hline Ercilla & Phytolaccaceae & 2 \\
\hline Erechtites & Asteraceae & 6 \\
\hline Eremium $(* *)$ & Poaceae & 1 \\
\hline Eremocharis & Apiaceae & 2 \\
\hline Eriachaenium & Asteraceae & 1 \\
\hline Erigeron & Asteraceae & 25 \\
\hline Eriobotrya (**) & Rosaceae & 1 \\
\hline Eriocaulon & Eriocaulaceae & 19 \\
\hline Eriochloa & Poaceae & 9 \\
\hline Eriochrysis & Poaceae & 5 \\
\hline Eriogonum & Polygonaceae & 1 \\
\hline Eriolarynx & Solanaceae & 2 \\
\hline Erioneuron & Poaceae & 2 \\
\hline Eriope & Lamiaceae & 2 \\
\hline Eriosema & Fabaceae & 15 \\
\hline Eriosyce & Cactaceae & 5 \\
\hline Eriotheca & Malvaceae & 2 \\
\hline Erodium & Geraniaceae & 5 \\
\hline Errazurizia & Fabaceae & 1 \\
\hline
\end{tabular}

\begin{tabular}{|c|c|c|}
\hline Género & Familia & $\mathbf{N}^{0} \mathrm{Sp}$. \\
\hline Eruca & Brassicaceae & 1 \\
\hline Eryngium & Apiaceae & 71 \\
\hline Erysimum & Brassicaceae & 2 \\
\hline Erythranthe (**) & Phrymaceae & 5 \\
\hline Erythrina & Fabaceae & 5 \\
\hline Erythrostemon (**) & Fabaceae & 7 \\
\hline Erythroxylum & Erythroxylaceae & 19 \\
\hline Escallonia & Escalloniaceae & 29 \\
\hline Eschscholzia & Papaveraceae & 1 \\
\hline Escobedia & Orobanchaceae & 1 \\
\hline Esenbeckia & Rutaceae & 5 \\
\hline Esterhazya & Orobanchaceae & 2 \\
\hline Eubrachion & Eremolepidaceae & 1 \\
\hline Eucalyptus & Myrtaceae & 7 \\
\hline Eucryphia & Cunoniaceae & 2 \\
\hline Eugenia & Myrtaceae & 109 \\
\hline Eulophia & Orchidaceae & 1 \\
\hline Eulychnia & Cactaceae & 8 \\
\hline Eumachia (**) & Rubiaceae & 5 \\
\hline Eupatorium & Asteraceae & 6 \\
\hline Euphorbia & Euphorbiaceae & 89 \\
\hline Euphrasia & Orobanchaceae & 15 \\
\hline Euplassa & Proteaceae & 2 \\
\hline Euploca $(* *)$ & Boraginaceae & 17 \\
\hline Eupodium (**) & Marattiaceae & 1 \\
\hline Euryops $(* *)$ & Asteraceae & 1 \\
\hline Eurystyles & Orchidaceae & 4 \\
\hline Eustachys & Poaceae & 9 \\
\hline Euterpe & Arecaceae & 1 \\
\hline Evolvulus & Convolvulaceae & 22 \\
\hline Exhalimolobos & Brassicaceae & 3 \\
\hline Exodeconus & Solanaceae & 3 \\
\hline Exostigma (*) & Asteraceae & 2 \\
\hline Exostyles & Fabaceae & 1 \\
\hline Fabiana & Solanaceae & 14 \\
\hline Facelis & Asteraceae & 3 \\
\hline Fagonia & Zygophyllaceae & 1 \\
\hline Fallopia (**) & Polygonaceae & 2 \\
\hline Famatina & Amaryllidaceae & 2 \\
\hline
\end{tabular}


Tabla 4. (Continuación).

\begin{tabular}{|c|c|c|c|c|c|}
\hline Género & Familia & $\mathrm{N}^{0} \mathrm{Sp}$. & Género & Familia & $\mathbf{N}^{0} \mathrm{Sp}$. \\
\hline Famatinanthus (*) & Asteraceae & 1 & Galium & Rubiaceae & 54 \\
\hline Faramea & Rubiaceae & 11 & Gallardoa & Malpighiaceae & 1 \\
\hline Fascicularia & Bromeliaceae & 1 & Gallesia & Phytolaccaceae & 1 \\
\hline Festuca & Poaceae & 57 & Galphimia & Malpighiaceae & 3 \\
\hline Fevillea & Cucurbitaceae & 2 & Gamocarpha & Calyceraceae & 16 \\
\hline Ficinia & Cyperaceae & 1 & Gamochaeta & Asteraceae & 43 \\
\hline Ficus & Moraceae & 24 & Garcinia & Clusiaceae & 3 \\
\hline Fimbristylis & Cyperaceae & 13 & Gardoquia (**) & Lamiaceae & 2 \\
\hline Fischeria & Apocynaceae & 1 & Gastoniella (*) & Pteridaceae & 1 \\
\hline Fitzroya & Cupressaceae & 1 & Gastridium & Poaceae & 2 \\
\hline Flaveria & Asteraceae & 2 & Gaudinia & Poaceae & 1 \\
\hline Fleischmannia (**) & Asteraceae & 7 & Gaultheria & Ericaceae & 21 \\
\hline Floscopa & Commelinaceae & 1 & Gavilea & Orchidaceae & 17 \\
\hline Flourensia & Asteraceae & 6 & Gaya & Malvaceae & 11 \\
\hline Foeniculum & Apiaceae & 1 & Gaylussacia & Ericaceae & 10 \\
\hline Fonkia & Plantaginaceae & 1 & Gayophytum & Onagraceae & 2 \\
\hline Forsteronia & Apocynaceae & 9 & Gazania & Asteraceae & 1 \\
\hline Fosterella & Bromeliaceae & 3 & Gelasine & Iridaceae & 4 \\
\hline Frailea & Cactaceae & 12 & Genipa & Rubiaceae & 1 \\
\hline Francoa & Francoaceae & 1 & Genista & Fabaceae & 2 \\
\hline Frankenia & Frankeniaceae & 10 & Genlisea & Lentibulariaceae & 2 \\
\hline Fraxinus & Oleaceae & 4 & Gentiana & Gentianaceae & 2 \\
\hline Freesia & Iridaceae & 2 & Gentianella & Gentianaceae & 31 \\
\hline Fridericia & Bignoniaceae & 16 & Geoffroea & Fabaceae & 2 \\
\hline Froelichia & Amaranthaceae & 4 & Geonoma & Arecaceae & 4 \\
\hline Fuchsia & Onagraceae & 6 & Geophila & Rubiaceae & 2 \\
\hline Fuirena & Cyperaceae & 3 & Geranium & Geraniaceae & 21 \\
\hline Fumaria & Papaveraceae & 8 & Gethyum & Amaryllidaceae & 2 \\
\hline Funastrum & Apocynaceae & 3 & Geum & Rosaceae & 8 \\
\hline Gaga (*) & Pteridaceae & 1 & Gevuina & Proteaceae & 1 \\
\hline Gaillardia & Asteraceae & 4 & Gibasis & Commelinaceae & 1 \\
\hline Gaimardia & Restionaceae & 1 & Gilia & Polemoniaceae & 3 \\
\hline Galactia & Fabaceae & 21 & Giliastrum & Polemoniaceae & 2 \\
\hline Galeandra & Orchidaceae & 5 & Gilliesia & Amaryllidaceae & 4 \\
\hline Galega & Fabaceae & 1 & Gladiolus & Iridaceae & 2 \\
\hline Galenia & Aizoaceae & 1 & Glandularia & Verbenaceae & 57 \\
\hline Galeopsis & Lamiaceae & 1 & Glaucium & Papaveraceae & 2 \\
\hline Galianthe & Rubiaceae & 32 & Glechoma & Lamiaceae & 1 \\
\hline Galinsoga & Asteraceae & 3 & Glechon & Lamiaceae & 5 \\
\hline
\end{tabular}


Tabla 4. (Continuación).

\begin{tabular}{|c|c|c|}
\hline Género & Familia & $\mathbf{N}^{0}$ Sp. \\
\hline Gleditsia & Fabaceae & 2 \\
\hline Gleichenella & Gleicheniaceae & 1 \\
\hline Glinus & Molluginaceae & 2 \\
\hline Gloxinia & Gesneriaceae & 2 \\
\hline Glyceria & Poaceae & 3 \\
\hline Glycyrrhiza & Fabaceae & 1 \\
\hline Gnaphalium & Asteraceae & 3 \\
\hline Gochnatia & Asteraceae & 5 \\
\hline Goeppertia (**) & Lauraceae & 2 \\
\hline Gomesa & Orchidaceae & 52 \\
\hline Gomortega & Gomortegaceae & 1 \\
\hline Gomphocarpus & Apocynaceae & 1 \\
\hline Gomphrena & Amaranthaceae & 39 \\
\hline Gongora & Orchidaceae & 2 \\
\hline Goniopteris (**) & Thelypteridaceae & 9 \\
\hline Gonolobus & Apocynaceae & 2 \\
\hline Gonopterodendron (*) & Zygophyllaceae & 2 \\
\hline Gordonia & Theaceae & 1 \\
\hline Gorgonidium & Araceae & 2 \\
\hline Gossypium & Malvaceae & 3 \\
\hline Gouania & Rhamnaceae & 4 \\
\hline Gouinia & Poaceae & 3 \\
\hline Govenia & Orchidaceae & 1 \\
\hline Grahamia & Anacampserotaceae & 1 \\
\hline Grammitis & Polypodiaceae & 1 \\
\hline Grandiphyllum (*) & Orchidaceae & 5 \\
\hline Graphistylis (**) & Asteraceae & 5 \\
\hline Gratiola & Plantaginaceae & 1 \\
\hline Grausa (*) & Loasaceae & 5 \\
\hline Grazielia (**) & Asteraceae & 8 \\
\hline Greigia & Bromeliaceae & 4 \\
\hline Grevillea & Proteaceae & 1 \\
\hline Grindelia & Asteraceae & 28 \\
\hline Griselinia & Griseliniaceae & 5 \\
\hline Grobya & Orchidaceae & 4 \\
\hline Guadua & Poaceae & 7 \\
\hline Guapira & Nyctaginaceae & 6 \\
\hline Guarea & Meliaceae & 3 \\
\hline Guatteria & Annonaceae & 1 \\
\hline
\end{tabular}

\begin{tabular}{|c|c|c|}
\hline Género & Familia & $\mathbf{N}^{0} \mathrm{Sp}$. \\
\hline Guazuma & Malvaceae & 1 \\
\hline Guettarda & Rubiaceae & 3 \\
\hline Guibourtia & Fabaceae & 1 \\
\hline Guilleminea & Amaranthaceae & 6 \\
\hline Guindilia & Sapindaceae & 3 \\
\hline Gunnera & Gunneraceae & 11 \\
\hline Gutierrezia & Asteraceae & 17 \\
\hline Guynesomia & Asteraceae & 1 \\
\hline Gymnanthemum (**) & Asteraceae & 1 \\
\hline Gymnanthes & Euphorbiaceae & 4 \\
\hline Gymneia (*) & Lamiaceae & 1 \\
\hline Gymnocalycium & Cactaceae & 60 \\
\hline Gymnocoronis & Asteraceae & 1 \\
\hline Gymnophyton & Apiaceae & 6 \\
\hline Gymnopogon & Poaceae & 6 \\
\hline Gymnosiphon & Burmanniaceae & 1 \\
\hline Gynerium & Poaceae & 1 \\
\hline Gynura & Asteraceae & 1 \\
\hline Gypothamnium & Asteraceae & 1 \\
\hline Gyptidium (**) & Asteraceae & 2 \\
\hline Gyptis (**) & Asteraceae & 5 \\
\hline Haageocereus & Cactaceae & 2 \\
\hline Habenaria & Orchidaceae & 69 \\
\hline Habranthus & Amaryllidaceae & 30 \\
\hline Hackelia & Boraginaceae & 1 \\
\hline Hackelochloa (**) & Poaceae & 1 \\
\hline Hagenbachia & Asparagaceae & 2 \\
\hline Hainardia & Poaceae & 1 \\
\hline Halenia & Gentianaceae & 1 \\
\hline Halerpestes & Ranunculaceae & 3 \\
\hline Halodule & Cymodoceaceae & 1 \\
\hline Halophytum & Halophytaceae & 1 \\
\hline Haloragis & Haloragaceae & 2 \\
\hline Halosicyos & Cucurbitaceae & 1 \\
\hline Hamadryas & Ranunculaceae & 5 \\
\hline Hamelia & Rubiaceae & 1 \\
\hline Hancornia & Apocynaceae & 1 \\
\hline Handroanthus (**) & Bignoniaceae & 11 \\
\hline Hapalorchis & Orchidaceae & 4 \\
\hline
\end{tabular}


Tabla 4. (Continuación).

\begin{tabular}{|c|c|c|}
\hline Género & Familia & $\mathbf{N}^{0}$ Sp. \\
\hline Haplopappus & Asteraceae & 64 \\
\hline Haplorhus & Anacardiaceae & 1 \\
\hline Haroldia (*) & Asteraceae & 1 \\
\hline Harrisia & Cactaceae & 5 \\
\hline Hatiora & Cactaceae & 3 \\
\hline Hatschbachiella (**) & Asteraceae & 2 \\
\hline Hebanthe & Amaranthaceae & 4 \\
\hline Hebe & Plantaginaceae & 1 \\
\hline Hebeclinium (**) & Asteraceae & 1 \\
\hline Hecistopteris & Pteridaceae & 1 \\
\hline Hedeoma & Lamiaceae & 5 \\
\hline Hedera & Araliaceae & 1 \\
\hline Hedychium & Zingiberaceae & 1 \\
\hline Hedyosmum & Lamiaceae & 1 \\
\hline Hedypnois & Asteraceae & 1 \\
\hline Heimia & Lythraceae & 3 \\
\hline Heisteria & Olacaceae & 2 \\
\hline Heladena & Malpighiaceae & 1 \\
\hline Helanthium (**) & Alismataceae & 2 \\
\hline Helenium & Asteraceae & 10 \\
\hline Helia & Gentianaceae & 2 \\
\hline Helianthus & Asteraceae & 4 \\
\hline Helichrysum (**) & Asteraceae & 1 \\
\hline Heliconia & Heliconiaceae & 5 \\
\hline Helicotropis (*) & Fabaceae & 1 \\
\hline Helicteres & Malvaceae & 3 \\
\hline Helictotrichon (**) & Poaceae & 1 \\
\hline Helietta & Rutaceae & 2 \\
\hline Heliocarpus & Malvaceae & 1 \\
\hline Heliotropium & Boraginaceae & 41 \\
\hline Helminthotheca $(* *)$ & Asteraceae & 1 \\
\hline Helogyne & Asteraceae & 3 \\
\hline Helosis & Balanophoraceae & 1 \\
\hline Hemarthria & Poaceae & 1 \\
\hline Hemidictyum & Hemidictyaceae & 1 \\
\hline Hemionitis & Pteridaceae & 1 \\
\hline Hemipogon & Apocynaceae & 2 \\
\hline Hennecartia & Monimiaceae & 1 \\
\hline Henriettea (**) & Melastomataceae & 1 \\
\hline
\end{tabular}

\begin{tabular}{|c|c|c|}
\hline Género & Familia & $\mathbf{N}^{0}$ Sp. \\
\hline Heracleum & Apiaceae & 1 \\
\hline Herbertia & Iridaceae & 9 \\
\hline Herbstia & Amaranthaceae & 1 \\
\hline Herissantia & Malvaceae & 3 \\
\hline Herniaria & Caryophyllaceae & 3 \\
\hline Herreria & Asparagaceae & 3 \\
\hline Hesperis & Brassicaceae & 1 \\
\hline Hesperozygis & Lamiaceae & 6 \\
\hline Heteranthera & Pontederiaceae & 6 \\
\hline Heterocentron (**) & Melastomataceae & 1 \\
\hline Heterocondylus (**) & Asteraceae & 8 \\
\hline Heterophyllaea & Rubiaceae & 1 \\
\hline Heteropogon & Poaceae & 2 \\
\hline Heteropsis & Araceae & 3 \\
\hline Heteropterys & Malpighiaceae & 28 \\
\hline Heterosperma & Asteraceae & 4 \\
\hline Heterostachys & Chenopodiaceae & 2 \\
\hline Heterothalamulopsis & Asteraceae & 1 \\
\hline Heterotheca & Asteraceae & 1 \\
\hline Heterotristicha & Podostemaceae & 1 \\
\hline Hevea & Euphorbiaceae & 1 \\
\hline Hexasepalum (**) & Rubiaceae & 3 \\
\hline Hibiscus & Malvaceae & 11 \\
\hline Hieracium & Asteraceae & 48 \\
\hline Hieronyma & Phyllanthaceae & 1 \\
\hline Hieronymiella & Amaryllidaceae & 6 \\
\hline Hieronymusia (**) & Saxifragaceae & 1 \\
\hline Hildaea (*) & Poaceae & 4 \\
\hline Hilleria & Phytolaccaceae & 1 \\
\hline Hillia & Rubiaceae & 3 \\
\hline Hindsia & Rubiaceae & 2 \\
\hline Hippeastrum & Amaryllidaceae & 21 \\
\hline Hippobroma & Campanulaceae & 1 \\
\hline Hippocratea & Celastraceae & 1 \\
\hline Hippuris & Plantaginaceae & 1 \\
\hline Hiraea & Malpighiaceae & 5 \\
\hline Hirschfeldia & Brassicaceae & 1 \\
\hline Hirtella & Chrysobalanaceae & 2 \\
\hline Histiopteris & Dennstaedtiaceae & 1 \\
\hline
\end{tabular}


Tabla 4. (Continuación).

\begin{tabular}{|c|c|c|}
\hline Género & Familia & $\mathbf{N}^{0}$ Sp. \\
\hline Hochreutinera & Malvaceae & 1 \\
\hline Hoehnea & Lamiaceae & 5 \\
\hline Hoehneella & Orchidaceae & 1 \\
\hline Hoffmannia & Rubiaceae & 2 \\
\hline Hoffmannseggia & Fabaceae & 11 \\
\hline Hohenbergia & Bromeliaceae & 1 \\
\hline Holcus & Poaceae & 1 \\
\hline Hollermayera & Brassicaceae & 1 \\
\hline Holmbergia & Chenopodiaceae & 1 \\
\hline Holocalyx & Fabaceae & 1 \\
\hline Holocheilus & Asteraceae & 7 \\
\hline Holosteum & Caryophyllaceae & 1 \\
\hline Homalocarpus & Apiaceae & 6 \\
\hline Homalolepis (**) & Simaroubaceae & 2 \\
\hline Homalopetalum (**) & Orchidaceae & 1 \\
\hline Homolepis & Poaceae & 2 \\
\hline Hordeum & Poaceae & 21 \\
\hline Hornungia & Brassicaceae & 1 \\
\hline Houlletia & Orchidaceae & 1 \\
\hline Houssayanthus & Sapindaceae & 2 \\
\hline Hovenia & Rhamnaceae & 1 \\
\hline Huarpea & Asteraceae & 1 \\
\hline Huberia & Melastomataceae & 1 \\
\hline Huidobria & Loasaceae & 2 \\
\hline Humulus & Cannabaceae & 2 \\
\hline Huntleya & Orchidaceae & 1 \\
\hline Huperzia & Lycopodiaceae & 4 \\
\hline Hyalis & Asteraceae & 2 \\
\hline Hyaloseris & Asteraceae & 4 \\
\hline Hybanthus & Violaceae & 2 \\
\hline Hydnocarpus & Achariaceae & 1 \\
\hline Hydrangea & Hydrangeaceae & 1 \\
\hline Hydrocleys & Alismataceae & 4 \\
\hline Hydrocotyle & Araliaceae & 19 \\
\hline Hydrolea & Hydroleaceae & 2 \\
\hline Hygrophila & Acanthaceae & 1 \\
\hline Hymenachne & Poaceae & 7 \\
\hline Hymenaea & Fabaceae & 3 \\
\hline Hymenasplenium (**) & Aspleniaceae & 2 \\
\hline
\end{tabular}

\begin{tabular}{|c|c|c|}
\hline Género & Familia & $\mathbf{N}^{0} \mathbf{S p}$. \\
\hline Hymenophyllum & Hymenophyllaceae & 41 \\
\hline Hymenostephium & Asteraceae & 1 \\
\hline Hymenoxys & Asteraceae & 4 \\
\hline Hyparrhenia & Poaceae & 2 \\
\hline Hypenia & Lamiaceae & 3 \\
\hline Hyperbaena & Menispermaceae & 3 \\
\hline Hypericum & Hypericaceae & 31 \\
\hline Hypochaeris & Asteraceae & 53 \\
\hline Hypoestes & Acanthaceae & 1 \\
\hline Hypolepis & Dennstaedtiaceae & 8 \\
\hline Hypolytrum & Cyperaceae & 1 \\
\hline Hypoxis & Hypoxidaceae & 4 \\
\hline Hypseocharis & Geraniaceae & 2 \\
\hline Hyptis & Lamiaceae & 35 \\
\hline Hysterionica & Asteraceae & 14 \\
\hline Ianthopappus & Asteraceae & 1 \\
\hline Ibatia (**) & Apocynaceae & 3 \\
\hline Iberis & Brassicaceae & 1 \\
\hline Ibicella & Martyniaceae & 2 \\
\hline Ichnanthus & Poaceae & 6 \\
\hline Ichthyothere & Asteraceae & 2 \\
\hline Idiothamnus (**) & Asteraceae & 2 \\
\hline Ilex & Aquifoliaceae & 12 \\
\hline Impatiens & Balsaminaceae & 3 \\
\hline Imperata & Poaceae & 6 \\
\hline Indigofera & Fabaceae & 13 \\
\hline Inga & Fabaceae & 18 \\
\hline Inulopsis & Asteraceae & 2 \\
\hline Iochroma & Solanaceae & 1 \\
\hline Ionopsis & Orchidaceae & 1 \\
\hline Ipheion & Amaryllidaceae & 4 \\
\hline Ipomoea & Convolvulaceae & 96 \\
\hline Ipomopsis & Polemoniaceae & 1 \\
\hline Iresine & Amaranthaceae & 1 \\
\hline Iris & Iridaceae & 4 \\
\hline Irlbachia & Gentianaceae & 1 \\
\hline Isabelia & Orchidaceae & 4 \\
\hline Isatis & Brassicaceae & 1 \\
\hline Ischaemum & Poaceae & 2 \\
\hline
\end{tabular}


Tabla 4. (Continuación).

\begin{tabular}{|c|c|c|}
\hline Género & Familia & $\mathbf{N}^{0}$ Sp. \\
\hline Ischnosiphon (**) & Marantaceae & 1 \\
\hline Islaya & Cactaceae & 4 \\
\hline Isochilus & Orchidaceae & 2 \\
\hline Isoetes & Isoetaceae & 21 \\
\hline Isolepis & Cyperaceae & 5 \\
\hline Isostigma & Asteraceae & 8 \\
\hline Ivania & Brassicaceae & 2 \\
\hline Ixora & Rubiaceae & 2 \\
\hline Ixorhea & Boraginaceae & 1 \\
\hline Jaborosa & Solanaceae & 22 \\
\hline Jacaranda & Bignoniaceae & 8 \\
\hline Jacaratia & Caricaceae & 2 \\
\hline Jacquemontia & Convolvulaceae & 25 \\
\hline Jacquiniella & Orchidaceae & 1 \\
\hline Jaegeria & Asteraceae & 1 \\
\hline Jamesonia $(* *)$ & Pteridaceae & 1 \\
\hline Janusia & Malpighiaceae & 4 \\
\hline Jarava & Poaceae & 26 \\
\hline Jasminum (**) & Oleaceae & 1 \\
\hline Jatropha & Euphorbiaceae & 18 \\
\hline Jaumea & Asteraceae & 1 \\
\hline Jobinia & Apocynaceae & 9 \\
\hline Jodina & Cervantesiaceae & 1 \\
\hline Johnstonella (**) & Boraginaceae & 1 \\
\hline Jovellana & Calceolariaceae & 3 \\
\hline Juania & Arecaceae & 1 \\
\hline Jubaea & Arecaceae & 1 \\
\hline Juglans & Juglandaceae & 1 \\
\hline Juncus & Juncaceae & 39 \\
\hline Junellia & Verbenaceae & 33 \\
\hline Jungia & Asteraceae & 5 \\
\hline Juniperus & Cupressaceae & 1 \\
\hline Justicia & Acanthaceae & 45 \\
\hline Kageneckia & Rosaceae & 3 \\
\hline Kalanchoe & Crassulaceae & 1 \\
\hline Kallstroemia & Zygophyllaceae & 2 \\
\hline Katinasia (*) & Asteraceae & 1 \\
\hline Kaunia $(* *)$ & Asteraceae & 3 \\
\hline Kentrothamnus & Rhamnaceae & 1 \\
\hline
\end{tabular}

\begin{tabular}{|c|c|c|}
\hline Género & Familia & $\mathbf{N}^{\circ}$ Sp. \\
\hline Kickxia & Plantaginaceae & 1 \\
\hline Kielmeyera & Calophyllaceae & 2 \\
\hline Kieslingia (*) & Asteraceae & 1 \\
\hline Knautia & Caprifoliaceae & 2 \\
\hline Kniphofia (**) & Asphodelaceae & 1 \\
\hline Koanophyllon (**) & Asteraceae & 5 \\
\hline Koeleria & Poaceae & 9 \\
\hline Koenigia & Polygonaceae & 1 \\
\hline Krameria & Krameriaceae & 3 \\
\hline Krapovickasia & Malvaceae & 3 \\
\hline Kummerowia (**) & Fabaceae & 1 \\
\hline Kurzamra & Lamiaceae & 1 \\
\hline Lablab & Fabaceae & 1 \\
\hline Laburnum & Fabaceae & 1 \\
\hline Lachemilla & Rosaceae & 8 \\
\hline Lachesiodendron (*) & Fabaceae & 1 \\
\hline Lachnagrostis & Poaceae & 1 \\
\hline Lacistema & Lacistemataceae & 2 \\
\hline Lactoris & Aristolochiaceae & 1 \\
\hline Lactuca & Asteraceae & 5 \\
\hline Laennecia & Asteraceae & 3 \\
\hline Laetia & Salicaceae & 1 \\
\hline Lafoensia & Lythraceae & 3 \\
\hline Lagascea & Asteraceae & 1 \\
\hline Lagenaria & Cucurbitaceae & 1 \\
\hline Lagenocarpus & Cyperaceae & 1 \\
\hline Lagenophora & Asteraceae & 3 \\
\hline Laguncularia & Combretaceae & 1 \\
\hline Lagurus & Poaceae & 1 \\
\hline Lamanonia & Cunoniaceae & 2 \\
\hline Lamarckia & Poaceae & 1 \\
\hline Lamium & Lamiaceae & 2 \\
\hline Lampayo & Verbenaceae & 3 \\
\hline Landoltia & Araceae & 1 \\
\hline Langsdorffia & Balanophoraceae & 2 \\
\hline Lankesterella & Orchidaceae & 3 \\
\hline Lantana & Verbenaceae & 19 \\
\hline Lapageria & Philesiaceae & 1 \\
\hline Laportea & Urticaceae & 1 \\
\hline
\end{tabular}


Tabla 4. (Continuación).

\begin{tabular}{|c|c|c|c|c|c|}
\hline Género & Familia & $\mathbf{N}^{0}$ Sp. & Género & Familia & $\mathbf{N}^{0}$ Sp. \\
\hline Lappula & Boraginaceae & 1 & Leptocarpha & Asteraceae & 1 \\
\hline Lapsana & Asteraceae & 1 & Leptochloa & Poaceae & 6 \\
\hline Lardizabala & Lardizabalaceae & 1 & Leptoglossis & Solanaceae & 1 \\
\hline Larrea & Zygophyllaceae & 4 & Leptophyllochloa & Poaceae & 1 \\
\hline Lasiacis & Poaceae & 3 & Leptosiphon & Polemoniaceae & 1 \\
\hline Lastarriaea & Polygonaceae & 1 & Leptospron (*) & Fabaceae & 1 \\
\hline Lasthenia & Asteraceae & 1 & Leptostelma & Asteraceae & 5 \\
\hline Lastreopsis & Dryopteridaceae & 1 & Leptostigma & Rubiaceae & 1 \\
\hline Latace (**) & Amaryllidaceae & 2 & Leptotes & Orchidaceae & 3 \\
\hline Lathyrus & Fabaceae & 33 & Lepturus (**) & Poaceae & 1 \\
\hline Latua & Solanaceae & 1 & Lepuropetalon & Celastraceae & 1 \\
\hline Laurelia & Monimiaceae & 1 & Lessingianthus (**) & Asteraceae & 50 \\
\hline Laureliopsis & Atherospermataceae & 1 & Leucaena & Fabaceae & 1 \\
\hline Laurembergia & Haloragaceae & 1 & Leucanthemum & Asteraceae & 1 \\
\hline Lavoisiera & Melastomataceae & 2 & Leucas (**) & Lamiaceae & 1 \\
\hline Leandra & Melastomataceae & 8 & Leucheria & Asteraceae & 47 \\
\hline Lebetanthus & Ericaceae & 1 & Leucochloron & Fabaceae & 1 \\
\hline Lecanophora & Malvaceae & 7 & Leucocoryne & Amaryllidaceae & 46 \\
\hline Lecointea $(* *)$ & Fabaceae & 1 & Leucojum (**) & Amaryllidaceae & 1 \\
\hline Leersia & Poaceae & 3 & Leucostele (**) & Cactaceae & 3 \\
\hline Legenere & Campanulaceae & 1 & Leucotrichum (*) & Polypodiaceae & 2 \\
\hline Legrandia & Myrtaceae & 1 & Leunisia & Asteraceae & 1 \\
\hline Leiothrix & Eriocaulaceae & 2 & Levisticum & Apiaceae & 1 \\
\hline Lellingeria & Polypodiaceae & 6 & Leymus & Poaceae & 1 \\
\hline Lemna & Araceae & 5 & Liabum & Asteraceae & 1 \\
\hline Lenzia & Montiaceae & 1 & Libertia & Iridaceae & 3 \\
\hline Leonotis & Lamiaceae & 1 & Libidibia $(* *)$ & Fabaceae & 1 \\
\hline Leontodon & Asteraceae & 3 & Licaria & Lauraceae & 1 \\
\hline Leonurus & Lamiaceae & 2 & Ligaria & Loranthaceae & 1 \\
\hline Lepanthopsis & Orchidaceae & 1 & Ligeophila & Orchidaceae & 2 \\
\hline Lepechinia & Lamiaceae & 6 & Ligustrum & Oleaceae & 4 \\
\hline Lepidagathis & Acanthaceae & 4 & Lilaeopsis & Apiaceae & 5 \\
\hline Lepidaploa & Asteraceae & 13 & Lilium (**) & Liliaceae & 1 \\
\hline Lepidium & Brassicaceae & 49 & Limnobium & Hydrocharitaceae & 1 \\
\hline Lepidoceras & Eremolepidaceae & 1 & Limnocharis & Alismataceae & 2 \\
\hline Lepidophyllum & Asteraceae & 1 & Limonium & Plumbaginaceae & 2 \\
\hline Lepidothamnus & Podocarpaceae & 1 & Limosella & Plantaginaceae & 1 \\
\hline Lepismium (**) & Cactaceae & 2 & Linaria & Plantaginaceae & 3 \\
\hline Leptinella & Asteraceae & 1 & Lindernia & Linderniaceae & 2 \\
\hline
\end{tabular}


Tabla 4. (Continuación).

\begin{tabular}{|c|c|c|}
\hline Género & Familia & $\mathbf{N}^{0}$ Sp. \\
\hline Lindsaea & Lindsaeaceae & 10 \\
\hline Linum & Linaceae & 15 \\
\hline Liparis & Orchidaceae & 2 \\
\hline Lippia & Verbenaceae & 41 \\
\hline Lithachne & Poaceae & 1 \\
\hline Lithodraba & Brassicaceae & 1 \\
\hline Lithospermum & Boraginaceae & 1 \\
\hline Lithraea & Anacardiaceae & 3 \\
\hline Littorella & Plantaginaceae & 1 \\
\hline Llagunoa & Sapindaceae & 1 \\
\hline Loasa & Loasaceae & 24 \\
\hline Lobelia & Campanulaceae & 20 \\
\hline Lobivia & Cactaceae & 23 \\
\hline Lobularia & Brassicaceae & 1 \\
\hline Lockhartia & Orchidaceae & 2 \\
\hline Loefgrenianthus & Orchidaceae & 1 \\
\hline Logfia & Asteraceae & 1 \\
\hline Lolium & Poaceae & 5 \\
\hline Lomanthus (*) & Asteraceae & 1 \\
\hline Lomaria $(* *)$ & Blechnaceae & 1 \\
\hline Lomaridium $(* *)$ & Blechnaceae & 3 \\
\hline Lomariocycas (*) & Blechnaceae & 5 \\
\hline Lomariopsis & Lomariopsidaceae & 1 \\
\hline Lomatia & Proteaceae & 3 \\
\hline Lonchocarpus & Fabaceae & 5 \\
\hline Lonicera & Caprifoliaceae & 1 \\
\hline Lophocarpinia & Fabaceae & 1 \\
\hline Lophopappus & Asteraceae & 3 \\
\hline Lophophytum & Balanophoraceae & 2 \\
\hline Lophosoria & Dicksoniaceae & 1 \\
\hline Lorentzianthus $(* *)$ & Asteraceae & 1 \\
\hline Lotus & Fabaceae & 7 \\
\hline Loudetia & Poaceae & 1 \\
\hline Loudetiopsis & Poaceae & 1 \\
\hline Louisiella $(* *)$ & Poaceae & 1 \\
\hline Loxopterygium & Anacardiaceae & 1 \\
\hline Lucilia & Asteraceae & 8 \\
\hline Ludwigia & Onagraceae & 32 \\
\hline Luehea & Malvaceae & 7 \\
\hline
\end{tabular}

\begin{tabular}{|c|c|c|}
\hline Género & Familia & $\mathrm{N}^{\circ} \mathrm{Sp}$. \\
\hline Luetzelburgia & Fabaceae & 1 \\
\hline Luffa & Cucurbitaceae & 1 \\
\hline Lulia & Asteraceae & 1 \\
\hline Luma & Myrtaceae & 2 \\
\hline Lunaria & Brassicaceae & 1 \\
\hline Lundia & Bignoniaceae & 2 \\
\hline Lupinus & Fabaceae & 45 \\
\hline Luziola & Poaceae & 7 \\
\hline Luzula & Juncaceae & 17 \\
\hline Luzuriaga & Alstroemeriaceae & 3 \\
\hline Lycapsus & Asteraceae & 1 \\
\hline Lychnis & Caryophyllaceae & 1 \\
\hline Lycianthes & Solanaceae & 6 \\
\hline Lycium & Solanaceae & 34 \\
\hline Lycopodiella & Lycopodiaceae & 4 \\
\hline Lycopodium & Lycopodiaceae & 1 \\
\hline Lycopsis & Boraginaceae & 1 \\
\hline Lycopus & Lamiaceae & 1 \\
\hline Lygodium & Lygodiaceae & 2 \\
\hline Lyroglossa & Orchidaceae & 1 \\
\hline Lysimachia & Primulaceae & 4 \\
\hline Lythrum & Lythraceae & 5 \\
\hline Lytoneuron (*) & Pteridaceae & 2 \\
\hline Mabea & Euphorbiaceae & 1 \\
\hline Macairea & Melastomataceae & 1 \\
\hline Machaerina & Cyperaceae & 4 \\
\hline Machaerium & Fabaceae & 15 \\
\hline Machaonia & Rubiaceae & 3 \\
\hline Maclura & Moraceae & 1 \\
\hline Macrachaenium & Asteraceae & 1 \\
\hline Macradenia & Orchidaceae & 1 \\
\hline Macrocarpaea & Gentianaceae & 1 \\
\hline Macropharynx & Apocynaceae & 3 \\
\hline Macropodina $(* *)$ & Asteraceae & 2 \\
\hline Macroptilium & Fabaceae & 14 \\
\hline Macroscepis & Apocynaceae & 5 \\
\hline Macrothelypteris & Thelypteridaceae & 1 \\
\hline Madia & Asteraceae & 2 \\
\hline Magnolia (**) & Magnoliaceae & 1 \\
\hline
\end{tabular}


Tabla 4. (Continuación).

\begin{tabular}{|c|c|c|}
\hline Género & Familia & $\mathbf{N}^{0}$ Sp. \\
\hline Magonia & Sapindaceae & 1 \\
\hline Maihuenia & Cactaceae & 2 \\
\hline Maihueniopsis & Cactaceae & 16 \\
\hline Maireana & Chenopodiaceae & 1 \\
\hline Malachra & Malvaceae & 1 \\
\hline Malacothrix & Asteraceae & 2 \\
\hline Malanea & Rubiaceae & 2 \\
\hline Malaxis & Orchidaceae & 13 \\
\hline Malcolmia & Brassicaceae & 1 \\
\hline Malesherbia & Malesherbiaceae & 16 \\
\hline Malmeanthus (**) & Asteraceae & 2 \\
\hline Malouetia $(* *)$ & Apocynaceae & 1 \\
\hline Malus & Rosaceae & 2 \\
\hline Malva & Malvaceae & 8 \\
\hline Malvastrum & Malvaceae & 10 \\
\hline Malvella & Malvaceae & 1 \\
\hline Mancoa & Brassicaceae & 3 \\
\hline Mandevilla & Apocynaceae & 23 \\
\hline Manekia (**) & Piperaceae & 1 \\
\hline Manettia & Rubiaceae & 12 \\
\hline Mangifera & Anacardiaceae & 1 \\
\hline Mangonia & Araceae & 2 \\
\hline Manihot & Euphorbiaceae & 19 \\
\hline Manilkara & Sapotaceae & 2 \\
\hline Mansoa & Bignoniaceae & 1 \\
\hline Maprounea & Euphorbiaceae & 2 \\
\hline Maranta & Marantaceae & 3 \\
\hline Marathrum & Podostemaceae & 1 \\
\hline Marattia & Marattiaceae & 1 \\
\hline Marcetia (**) & Melastomataceae & 1 \\
\hline Marcgravia & Marcgraviaceae & 1 \\
\hline Margaritaria & Phyllanthaceae & 1 \\
\hline Margyracaena & Rosaceae & 1 \\
\hline Margyricarpus & Rosaceae & 2 \\
\hline Marlierea & Myrtaceae & 3 \\
\hline Marrubium & Lamiaceae & 1 \\
\hline Marsdenia & Apocynaceae & 4 \\
\hline Marsilea & Marsileaceae & 4 \\
\hline Marsippospermum & Juncaceae & 3 \\
\hline
\end{tabular}

\begin{tabular}{|c|c|c|}
\hline Género & Familia & $\mathbf{N}^{0}$ Sp. \\
\hline Marsypianthes & Lamiaceae & 2 \\
\hline Marticorenia & Asteraceae & 1 \\
\hline Mascagnia & Malpighiaceae & 4 \\
\hline Masdevallia & Orchidaceae & 1 \\
\hline Mastigostyla & Iridaceae & 8 \\
\hline Matayba & Sapindaceae & 7 \\
\hline Matelea & Apocynaceae & 15 \\
\hline Mathewsia & Brassicaceae & 4 \\
\hline Matricaria & Asteraceae & 2 \\
\hline Matthiola & Brassicaceae & 1 \\
\hline Maurandya & Plantaginaceae & 3 \\
\hline Mauria & Anacardiaceae & 1 \\
\hline Maxillaria & Orchidaceae & 43 \\
\hline Mayaca & Mayacaceae & 3 \\
\hline Maytenus & Celastraceae & 7 \\
\hline Mazus & Mazaceae & 1 \\
\hline Mecardonia & Plantaginaceae & 7 \\
\hline Medicago & Fabaceae & 10 \\
\hline Medusantha (*) & Lamiaceae & 1 \\
\hline Megalachne & Poaceae & 3 \\
\hline Megalastrum & Dryopteridaceae & 17 \\
\hline Megathyrsus & Poaceae & 1 \\
\hline Melampodium (**) & Asteraceae & 1 \\
\hline Melananthus & Solanaceae & 1 \\
\hline Melanophora & Aizoaceae & 1 \\
\hline Melanthera & Asteraceae & 1 \\
\hline Melasma & Orobanchaceae & 3 \\
\hline Melia & Meliaceae & 1 \\
\hline Melica & Poaceae & 30 \\
\hline Melicoccus & Sapindaceae & 1 \\
\hline Melidiscus (**) & Cleomaceae & 1 \\
\hline Melilotus & Fabaceae & 4 \\
\hline Melinis & Poaceae & 2 \\
\hline Meliosma & Sabiaceae & 1 \\
\hline Melissa & Lamiaceae & 1 \\
\hline Melochia & Malvaceae & 18 \\
\hline Melosperma & Plantaginaceae & 1 \\
\hline Melothria & Cucurbitaceae & 8 \\
\hline Melothrianthus (**) & Cucurbitaceae & 1 \\
\hline
\end{tabular}


Tabla 4. (Continuación).

\begin{tabular}{|c|c|c|}
\hline Género & Familia & $\mathbf{N}^{0}$ Sp. \\
\hline Melpomene & Polypodiaceae & 4 \\
\hline Mendoncia & Acanthaceae & 2 \\
\hline Meniscium (**) & Thelypteridaceae & 5 \\
\hline Menodora & Oleaceae & 5 \\
\hline Menonvillea & Brassicaceae & 24 \\
\hline Mentha & Lamiaceae & 5 \\
\hline Mentzelia & Loasaceae & 5 \\
\hline Mercurialis & Euphorbiaceae & 1 \\
\hline Merostachys & Poaceae & 15 \\
\hline Mesadenella & Orchidaceae & 2 \\
\hline Mesanthophora (**) & Asteraceae & 2 \\
\hline Mesechites & Apocynaceae & 2 \\
\hline Mesembryanthemum & Aizoaceae & 2 \\
\hline Mesosetum & Poaceae & 5 \\
\hline Mesosphaerum (**) & Lamiaceae & 3 \\
\hline Metalepis $(* *)$ & Apocynaceae & 1 \\
\hline Metastelma & Apocynaceae & 3 \\
\hline Metharme & Zygophyllaceae & 1 \\
\hline Metrodorea & Rutaceae & 1 \\
\hline Metrosideros (**) & Myrtaceae & 1 \\
\hline Mickelia (*) & Dryopteridaceae & 1 \\
\hline Miconia & Melastomataceae & 123 \\
\hline Micranthemum & Linderniaceae & 1 \\
\hline Microchilus & Orchidaceae & 3 \\
\hline Microchloa & Poaceae & 2 \\
\hline Microgramma & Polypodiaceae & 8 \\
\hline Microgyne & Asteraceae & 2 \\
\hline Microlepia & Dennstaedtiaceae & 2 \\
\hline Microliabum & Asteraceae & 6 \\
\hline Microlicia & Melastomataceae & 1 \\
\hline Microlobius & Fabaceae & 1 \\
\hline Microphyes & Caryophyllaceae & 3 \\
\hline Micropsis & Asteraceae & 5 \\
\hline Microseris & Asteraceae & 1 \\
\hline Microsorum (**) & Polypodiaceae & 1 \\
\hline Microstachys & Euphorbiaceae & 6 \\
\hline Microsteris & Polemoniaceae & 1 \\
\hline Microtea & Microteaceae & 5 \\
\hline Miersia & Amaryllidaceae & 4 \\
\hline
\end{tabular}

\begin{tabular}{|c|c|c|}
\hline Género & Familia & $\mathbf{N}^{0}$ Sp. \\
\hline Miersiella & Burmanniaceae & 1 \\
\hline Mikania & Asteraceae & 86 \\
\hline Miltonia & Orchidaceae & 4 \\
\hline Mimosa & Fabaceae & 177 \\
\hline Mimozyganthus & Fabaceae & 1 \\
\hline Minaria & Apocynaceae & 1 \\
\hline Minthostachys & Lamiaceae & 2 \\
\hline Mionandra & Malpighiaceae & 2 \\
\hline Mirabilis & Nyctaginaceae & 9 \\
\hline Miscanthus & Poaceae & 1 \\
\hline Misodendrum & Misodendraceae & 8 \\
\hline Misopates (**) & Plantaginaceae & 1 \\
\hline Mitracarpus & Rubiaceae & 8 \\
\hline Mitraria & Gesneriaceae & 1 \\
\hline Mnesithea & Poaceae & 5 \\
\hline Mniodes & Asteraceae & 9 \\
\hline Modiola & Malvaceae & 1 \\
\hline Modiolastrum & Malvaceae & 5 \\
\hline Molineria & Hypoxidaceae & 1 \\
\hline Mollinedia & Monimiaceae & 15 \\
\hline Mollugo & Molluginaceae & 2 \\
\hline Molucella & Lamiaceae & 1 \\
\hline Momordica & Cucurbitaceae & 1 \\
\hline Monnina & Polygalaceae & 19 \\
\hline Monopera (**) & Plantaginaceae & 1 \\
\hline Monstera & Araceae & 2 \\
\hline Montanoa & Asteraceae & 1 \\
\hline Monteiroa & Malvaceae & 9 \\
\hline Monteverdia (**) & Celastraceae & 14 \\
\hline Montia & Montiaceae & 1 \\
\hline Montiopsis & Montiaceae & 18 \\
\hline Monttea & Plantaginaceae & 3 \\
\hline Monvillea & Cactaceae & 5 \\
\hline Moorochloa & Poaceae & 1 \\
\hline Moquiniastrum (*) & Asteraceae & 13 \\
\hline Moranopteris (*) & Polypodiaceae & 3 \\
\hline Morella & Myricaceae & 2 \\
\hline Moritzia & Boraginaceae & 2 \\
\hline Morrenia & Apocynaceae & 2 \\
\hline
\end{tabular}


Tabla 4. (Continuación).

\begin{tabular}{|c|c|c|}
\hline Género & Familia & $\mathbf{N}^{o}$ Sp. \\
\hline Morronea (*) & Poaceae & 1 \\
\hline Morus & Moraceae & 2 \\
\hline Moscharia & Asteraceae & 2 \\
\hline Moschopis & Calyceraceae & 7 \\
\hline Mostacillastrum & Brassicaceae & 16 \\
\hline Mourera & Podostemaceae & 2 \\
\hline Mouriri & Melastomataceae & 2 \\
\hline Mucuna & Fabaceae & 3 \\
\hline Muehlenbeckia & Polygonaceae & 4 \\
\hline Muellera $(* *)$ & Fabaceae & 7 \\
\hline Muhlenbergia & Poaceae & 19 \\
\hline Mulguraea (*) & Verbenaceae & 11 \\
\hline Munnozia & Asteraceae & 1 \\
\hline Munroa & Poaceae & 4 \\
\hline Muntingia & Muntingiaceae & 1 \\
\hline Murdannia (**) & Commelinaceae & 1 \\
\hline Musa $(* *)$ & Musaceae & 2 \\
\hline Mutisia & Asteraceae & 35 \\
\hline Myoporum & Scrophulariaceae & 1 \\
\hline Myoschilos & Santalaceae & 1 \\
\hline Myosotis & Boraginaceae & 12 \\
\hline Myostemma (**) & Amaryllidaceae & 10 \\
\hline Myosurus & Ranunculaceae & 2 \\
\hline Myoxanthus & Orchidaceae & 1 \\
\hline Myracrodruon (**) & Anacardiaceae & 2 \\
\hline Myrceugenia & Myrtaceae & 44 \\
\hline Myrcia & Myrtaceae & 68 \\
\hline Myrcianthes & Myrtaceae & 10 \\
\hline Myrciaria & Myrtaceae & 7 \\
\hline Myriocarpa & Urticaceae & 1 \\
\hline Myriophyllum & Haloragaceae & 2 \\
\hline Myriopteris (**) & Pteridaceae & 4 \\
\hline Myriopus (**) & Boraginaceae & 5 \\
\hline Myrocarpus & Fabaceae & 1 \\
\hline Myrosmodes (**) & Orchidaceae & 3 \\
\hline Myroxylon & Fabaceae & 1 \\
\hline Myrrhinium & Myrtaceae & 1 \\
\hline Myrsine & Primulaceae & 19 \\
\hline Myrteola & Myrtaceae & 1 \\
\hline
\end{tabular}

\begin{tabular}{|c|c|c|}
\hline Género & Familia & $\mathbf{N}^{0}$ Sp. \\
\hline Najas & Hydrocharitaceae & 4 \\
\hline Nama & Boraginaceae & 3 \\
\hline Nanodea & Nanodaceae & 1 \\
\hline Napeanthus & Gesneriaceae & 2 \\
\hline Nardophyllum & Asteraceae & 5 \\
\hline Nasa & Loasaceae & 1 \\
\hline Nassauvia & Asteraceae & 39 \\
\hline Nassella & Poaceae & 97 \\
\hline Nasturtium & Brassicaceae & 2 \\
\hline Nautonia & Apocynaceae & 1 \\
\hline Navarretia & Polemoniaceae & 1 \\
\hline Nectandra & Lauraceae & 17 \\
\hline Neea & Nyctaginaceae & 2 \\
\hline Nelumbo $(* *)$ & Nymphaeaceae & 1 \\
\hline Nemaconia (**) & Orchidaceae & 1 \\
\hline Nematanthus & Gesneriaceae & 6 \\
\hline Neobaclea & Malvaceae & 1 \\
\hline Neobartsia (*) & Orobanchaceae & 9 \\
\hline Neoblechnum (*) & Blechnaceae & 1 \\
\hline Neobouteloua & Poaceae & 2 \\
\hline Neocabreria (**) & Asteraceae & 5 \\
\hline Neomarica & Iridaceae & 9 \\
\hline Neomitranthes & Myrtaceae & 6 \\
\hline Neonotonia & Fabaceae & 1 \\
\hline Neoporteria & Cactaceae & 6 \\
\hline Neoregelia & Bromeliaceae & 3 \\
\hline Neosparton & Verbenaceae & 3 \\
\hline Neowerdermannia & Cactaceae & 2 \\
\hline Nepeta & Lamiaceae & 1 \\
\hline Nephrolepis & Nephrolepidaceae & 7 \\
\hline Neptunia & Fabaceae & 2 \\
\hline Nertera & Rubiaceae & 1 \\
\hline Neslia & Brassicaceae & 1 \\
\hline Nesocaryum & Boraginaceae & 1 \\
\hline Neuontobotrys & Brassicaceae & 12 \\
\hline Nicandra & Solanaceae & 1 \\
\hline Nicoraepoa (*) & Poaceae & 6 \\
\hline Nicotiana & Solanaceae & 27 \\
\hline Nidularium & Bromeliaceae & 17 \\
\hline
\end{tabular}


Tabla 4. (Continuación).

\begin{tabular}{|c|c|c|}
\hline Género & Familia & $\mathbf{N}^{0} \mathrm{Sp}$. \\
\hline Niedenzuella & Malpighiaceae & 6 \\
\hline Nierembergia & Solanaceae & 18 \\
\hline Niphidium & Polypodiaceae & 2 \\
\hline Nissolia & Fabaceae & 6 \\
\hline Nitrophila & Chenopodiaceae & 2 \\
\hline Noccaea & Brassicaceae & 1 \\
\hline Noisettia & Violaceae & 1 \\
\hline Nolana & Solanaceae & 51 \\
\hline Nopalea (**) & Cactaceae & 1 \\
\hline Norantea (**) & Marcgraviaceae & 2 \\
\hline Nordenstamia $(* *)$ & Asteraceae & 1 \\
\hline Notanthera & Loranthaceae & 1 \\
\hline Nothofagus & Nothofagaceae & 10 \\
\hline Notholaena & Pteridaceae & 1 \\
\hline Nothoschkuhria (*) & Asteraceae & 1 \\
\hline Nothoscordum & Amaryllidaceae & 75 \\
\hline Noticastrum & Asteraceae & 19 \\
\hline Notiosciadium & Apiaceae & 1 \\
\hline Notogrammitis (*) & Polypodiaceae & 3 \\
\hline Nototriche & Malvaceae & 49 \\
\hline Notylia & Orchidaceae & 3 \\
\hline Novenia & Asteraceae & 1 \\
\hline Nuttallanthus (**) & Plantaginaceae & 1 \\
\hline Nymphaea & Nymphaeaceae & 8 \\
\hline Nymphoides & Menyanthaceae & 2 \\
\hline Ocellochloa $(*)$ & Poaceae & 2 \\
\hline Ochagavia & Bromeliaceae & 4 \\
\hline Ochetophila & Rhamnaceae & 2 \\
\hline Ocimum & Lamiaceae & 6 \\
\hline Ocotea & Lauraceae & 40 \\
\hline Octomeria & Orchidaceae & 38 \\
\hline Ocyroe (**) & Asteraceae & 1 \\
\hline Odonellia (**) & Convolvulaceae & 1 \\
\hline Odontocarya & Menispermaceae & 3 \\
\hline Odontonema & Acanthaceae & 1 \\
\hline Odontorrhynchus & Orchidaceae & 2 \\
\hline Oeceoclades & Orchidaceae & 1 \\
\hline Oedochloa (*) & Poaceae & 2 \\
\hline Oenanthe & Apiaceae & 2 \\
\hline
\end{tabular}

\begin{tabular}{|c|c|c|}
\hline Género & Familia & $\mathbf{N}^{0}$ Sp. \\
\hline Oenothera & Onagraceae & 41 \\
\hline Oldenlandia & Rubiaceae & 5 \\
\hline Oleandra (**) & Oleandraceae & 1 \\
\hline Olfersia & Dryopteridaceae & 1 \\
\hline Oligocladus & Apiaceae & 1 \\
\hline Olsynium & Iridaceae & 13 \\
\hline Olyra & Poaceae & 5 \\
\hline Ombrophytum & Balanophoraceae & 1 \\
\hline Omphalodes & Boraginaceae & 1 \\
\hline Oncidium & Orchidaceae & 4 \\
\hline Oncorachis (*) & Poaceae & 1 \\
\hline Onira $(* *)$ & Iridaceae & 1 \\
\hline Ononis & Fabaceae & 1 \\
\hline Onopordum & Asteraceae & 2 \\
\hline Onoseris & Asteraceae & 2 \\
\hline Onuris & Brassicaceae & 5 \\
\hline Oocephalus (*) & Lamiaceae & 1 \\
\hline Operculina & Convolvulaceae & 1 \\
\hline Ophioglossum & Ophioglossaceae & 7 \\
\hline Ophiopogon (**) & Asparagaceae & 1 \\
\hline Ophryosporus & Asteraceae & 16 \\
\hline Ophthalmoblapton (**) & Euphorbiaceae & 1 \\
\hline Oplismenopsis & Poaceae & 1 \\
\hline Oplismenus & Poaceae & 1 \\
\hline Oplonia & Acanthaceae & 1 \\
\hline Opuntia & Cactaceae & 19 \\
\hline Oreobolus & Cyperaceae & 1 \\
\hline Oreocereus & Cactaceae & 4 \\
\hline Oreopanax & Araliaceae & 3 \\
\hline Oreopolus & Rubiaceae & 1 \\
\hline Oriastrum (**) & Asteraceae & 16 \\
\hline Origanum (**) & Lamiaceae & 1 \\
\hline Orites & Proteaceae & 1 \\
\hline Ormosia & Fabaceae & 2 \\
\hline Ornithocephalus & Orchidaceae & 2 \\
\hline Ornithopus & Fabaceae & 4 \\
\hline Orobanche & Orobanchaceae & 4 \\
\hline Ortachne & Poaceae & 2 \\
\hline Orthochilus (**) & Orchidaceae & 1 \\
\hline
\end{tabular}


Tabla 4. (Continuación).

\begin{tabular}{|c|c|c|}
\hline Género & Familia & $\mathbf{N}^{0}$ Sp. \\
\hline Orthoclada & Poaceae & 1 \\
\hline Orthopappus & Asteraceae & 1 \\
\hline Orthosia & Apocynaceae & 8 \\
\hline Orthrosanthus & Iridaceae & 1 \\
\hline Oryza & Poaceae & 3 \\
\hline Osmia (**) & Asteraceae & 1 \\
\hline Osmorhiza & Apiaceae & 4 \\
\hline Osmunda & Osmundaceae & 2 \\
\hline Osmundastrum (**) & Osmundaceae & 1 \\
\hline Ossaea & Melastomataceae & 1 \\
\hline Osvaldoa (*) & Poaceae & 1 \\
\hline Otachyrium & Poaceae & 1 \\
\hline Otholobium & Fabaceae & 3 \\
\hline Ottelia & Hydrocharitaceae & 1 \\
\hline Ouratea & Ochnaceae & 8 \\
\hline Ourisia & Plantaginaceae & 12 \\
\hline Ovidia & Thymelaeaceae & 2 \\
\hline Oxalis & Oxalidaceae & 122 \\
\hline Oxossia $(*)$ & Turneraceae & 1 \\
\hline Oxybasis (**) & Chenopodiaceae & 4 \\
\hline Oxycaryum & Cyperaceae & 1 \\
\hline Oxychloe & Juncaceae & 5 \\
\hline Oxypetalum & Apocynaceae & 85 \\
\hline Oxyphyllum & Asteraceae & 1 \\
\hline Oxytheca & Polygonaceae & 1 \\
\hline Ozirö̈ & Asparagaceae & 5 \\
\hline Pabstia & Orchidaceae & 2 \\
\hline Pabstiella & Orchidaceae & 41 \\
\hline Pachira (**) & Malvaceae & 1 \\
\hline Pachylaena & Asteraceae & 1 \\
\hline Pachyrhizus & Fabaceae & 1 \\
\hline Pachystachys (**) & Acanthaceae & 2 \\
\hline Pachystroma & Euphorbiaceae & 1 \\
\hline Pacourina & Asteraceae & 1 \\
\hline Paederia & Rubiaceae & 1 \\
\hline Paepalanthus & Eriocaulaceae & 14 \\
\hline Paesia & Dennstaedtiaceae & 1 \\
\hline Palaua & Malvaceae & 4 \\
\hline Palhinhaea (**) & Lycopodiaceae & 2 \\
\hline
\end{tabular}

\begin{tabular}{|c|c|c|}
\hline Género & Familia & $\mathbf{N}^{0}$ Sp. \\
\hline Palicourea & Rubiaceae & 10 \\
\hline Pamphalea (**) & Asteraceae & 10 \\
\hline Panicum & Poaceae & 32 \\
\hline Pantacantha & Solanaceae & 1 \\
\hline Papaver & Papaveraceae & 5 \\
\hline Pappophorum & Poaceae & 7 \\
\hline Pappostipa (*) & Poaceae & 29 \\
\hline Parablechnum (**) & Blechnaceae & 3 \\
\hline Paradisanthus & Orchidaceae & 2 \\
\hline Paranephelius & Asteraceae & 1 \\
\hline Parapholis & Poaceae & 2 \\
\hline Parapiptadenia & Fabaceae & 2 \\
\hline Parapolystichum (**) & Dryopteridaceae & 1 \\
\hline Parasenegalia $(*)$ & Fabaceae & 1 \\
\hline Parastrephia & Asteraceae & 4 \\
\hline Parentucellia & Orobanchaceae & 1 \\
\hline Parietaria & Urticaceae & 3 \\
\hline Parinari & Chrysobalanaceae & 1 \\
\hline Parkinsonia & Fabaceae & 3 \\
\hline Parodia & Cactaceae & 42 \\
\hline Parodianthus & Verbenaceae & 2 \\
\hline Parodiodendron & Picrodendraceae & 1 \\
\hline Parodiodoxa & Brassicaceae & 1 \\
\hline Parodiolyra & Poaceae & 1 \\
\hline Parodiophyllochloa (*) & Poaceae & 5 \\
\hline Paronychia & Caryophyllaceae & 13 \\
\hline Parthenium & Asteraceae & 2 \\
\hline Parthenocissus (**) & Vitaceae & 2 \\
\hline Pascalia $(* *)$ & Asteraceae & 2 \\
\hline Pasithea & Asphodelaceae & 1 \\
\hline Paspalum & Poaceae & 115 \\
\hline Passiflora & Passifloraceae & 43 \\
\hline Pastinaca & Apiaceae & 1 \\
\hline Patosia & Juncaceae & 1 \\
\hline Paubrasilia (*) & Fabaceae & 1 \\
\hline Paullinia & Sapindaceae & 11 \\
\hline Pausandra & Euphorbiaceae & 1 \\
\hline Pavonia & Malvaceae & 77 \\
\hline Pecluma & Polypodiaceae & 15 \\
\hline
\end{tabular}


Tabla 4. (Continuación).

\begin{tabular}{|c|c|c|}
\hline Género & Familia & $\mathbf{N}^{0}$ Sp. \\
\hline Pectis & Asteraceae & 4 \\
\hline Pectocarya & Boraginaceae & 5 \\
\hline Pedersenia & Amaranthaceae & 2 \\
\hline Peixotoa & Malpighiaceae & 5 \\
\hline Pelexia & Orchidaceae & 35 \\
\hline Pellaea & Pteridaceae & 4 \\
\hline Pelletiera & Primulaceae & 1 \\
\hline Peltaea & Malvaceae & 5 \\
\hline Peltophorum & Fabaceae & 1 \\
\hline Peltophyllum & Triuridaceae & 1 \\
\hline Pennellia & Brassicaceae & 3 \\
\hline Pentacalia (**) & Asteraceae & 2 \\
\hline Peperomia & Piperaceae & 100 \\
\hline Peplonia & Apocynaceae & 3 \\
\hline Pera & Peraceae & 2 \\
\hline Pereskia & Cactaceae & 4 \\
\hline Perezia & Asteraceae & 30 \\
\hline Periandra & Fabaceae & 1 \\
\hline Perianthomega & Bignoniaceae & 1 \\
\hline Peritassa & Celastraceae & 2 \\
\hline Perityle & Asteraceae & 1 \\
\hline Persea & Lauraceae & 8 \\
\hline Persicaria (**) & Polygonaceae & 1 \\
\hline Petalostelma & Apocynaceae & 2 \\
\hline Petiveria & Phytolaccaceae & 1 \\
\hline Petrea & Verbenaceae & 1 \\
\hline Petroravenia & Brassicaceae & 1 \\
\hline Petrorhagia & Caryophyllaceae & 3 \\
\hline Petroselinum & Apiaceae & 1 \\
\hline Petunia & Solanaceae & 14 \\
\hline Peumus & Monimiaceae & 1 \\
\hline Pfaffia & Amaranthaceae & 11 \\
\hline Pfeiffera & Cactaceae & 1 \\
\hline Phacelia & Boraginaceae & 9 \\
\hline Phaeostemma (**) & Apocynaceae & 2 \\
\hline Phalaris & Poaceae & 10 \\
\hline Pharus & Poaceae & 2 \\
\hline Phaseolus & Fabaceae & 4 \\
\hline Phemeranthus (**) & Montiaceae & 1 \\
\hline
\end{tabular}

\begin{tabular}{|c|c|c|}
\hline Género & Familia & $\mathbf{N}^{0}$ Sp. \\
\hline Phenax & Urticaceae & 2 \\
\hline Philesia & Philesiaceae & 1 \\
\hline Philibertia & Apocynaceae & 30 \\
\hline Philippiella & Caryophyllaceae & 1 \\
\hline Philodendron & Araceae & 18 \\
\hline Philyra & Euphorbiaceae & 1 \\
\hline Phippsia & Poaceae & 1 \\
\hline Phlebodium & Polypodiaceae & 3 \\
\hline Phlebolobium & Brassicaceae & 1 \\
\hline Phlegmariurus (**) & Lycopodiaceae & 22 \\
\hline Phleum & Poaceae & 2 \\
\hline Phloeophila & Orchidaceae & 2 \\
\hline Phoenix & Arecaceae & 1 \\
\hline Phoradendron & Viscaceae & 23 \\
\hline Phragmites & Poaceae & 1 \\
\hline Phycella & Amaryllidaceae & 6 \\
\hline Phyla & Verbenaceae & 2 \\
\hline Phyllachne & Stylidiaceae & 1 \\
\hline Phyllanthus & Phyllanthaceae & 24 \\
\hline Phyllitis & Aspleniaceae & 1 \\
\hline Phylloscirpus & Cyperaceae & 3 \\
\hline Phyllostylon & Ulmaceae & 1 \\
\hline Phymatidium & Orchidaceae & 5 \\
\hline Physalis & Solanaceae & 10 \\
\hline Physaria & Brassicaceae & 6 \\
\hline Phytolacca & Phytolaccaceae & 7 \\
\hline Picradeniopsis (**) & Asteraceae & 1 \\
\hline Picramnia & Picramniaceae & 5 \\
\hline Picrasma & Simaroubaceae & 1 \\
\hline Picrosia & Asteraceae & 2 \\
\hline Pilea & Urticaceae & 13 \\
\hline Pilgerodendron & Cupressaceae & 1 \\
\hline Pilocarpus & Rutaceae & 3 \\
\hline Pilosocereus & Cactaceae & 1 \\
\hline Pilostyles & Apodanthaceae & 2 \\
\hline Pilularia & Marsileaceae & 1 \\
\hline Pimenta & Myrtaceae & 1 \\
\hline Pimpinella & Apiaceae & 1 \\
\hline Pinguicula & Lentibulariaceae & 4 \\
\hline
\end{tabular}


Tabla 4. (Continuación).

\begin{tabular}{|c|c|c|}
\hline Género & Familia & $\mathbf{N}^{0}$ Sp. \\
\hline Pinnasa (*) & Loasaceae & 4 \\
\hline Pintoa & Zygophyllaceae & 1 \\
\hline Pinus & Pinaceae & 13 \\
\hline Piper & Piperaceae & 58 \\
\hline Piptadenia & Fabaceae & 4 \\
\hline Piptadeniopsis & Fabaceae & 1 \\
\hline Piptatherum & Poaceae & 1 \\
\hline Piptocarpha & Asteraceae & 15 \\
\hline Piptochaetium & Poaceae & 25 \\
\hline Piriqueta & Turneraceae & 13 \\
\hline Pisonia & Nyctaginaceae & 3 \\
\hline Pisoniella & Nyctaginaceae & 1 \\
\hline Pistia & Araceae & 1 \\
\hline Pitavia & Rutaceae & 1 \\
\hline Pitcairnia & Bromeliaceae & 5 \\
\hline Pitraea & Verbenaceae & 1 \\
\hline Pittosporum (**) & Pittosporaceae & 2 \\
\hline Pityrogramma & Pteridaceae & 3 \\
\hline Placea & Amaryllidaceae & 5 \\
\hline Plagiobothrys & Boraginaceae & 19 \\
\hline Plagiocheilus & Asteraceae & 1 \\
\hline Plagiogyria (**) & Plagiogyriaceae & 1 \\
\hline Plantago & Plantaginaceae & 55 \\
\hline Plathymenia & Fabaceae & 1 \\
\hline Platycyamus (**) & Fabaceae & 1 \\
\hline Platymiscium & Fabaceae & 1 \\
\hline Platypodium & Fabaceae & 1 \\
\hline Platyrhiza & Orchidaceae & 1 \\
\hline Platystele & Orchidaceae & 1 \\
\hline Platythelys & Orchidaceae & 3 \\
\hline Plazia & Asteraceae & 2 \\
\hline Plectocephalus (**) & Asteraceae & 1 \\
\hline Plectrocarpa & Zygophyllaceae & 2 \\
\hline Plenckia & Celastraceae & 3 \\
\hline Pleocarphus & Asteraceae & 1 \\
\hline Pleopeltis & Polypodiaceae & 13 \\
\hline Pleroma (**) & Melastomataceae & 4 \\
\hline Pleurophora & Lythraceae & 5 \\
\hline Pleurostachys & Cyperaceae & 9 \\
\hline
\end{tabular}

\begin{tabular}{|c|c|c|}
\hline Género & Familia & $\mathbf{N}^{0} \mathrm{Sp}$. \\
\hline Plinia & Myrtaceae & 8 \\
\hline Pluchea & Asteraceae & 5 \\
\hline Plumbago & Plumbaginaceae & 2 \\
\hline Plumeria & Apocynaceae & 1 \\
\hline Poa & Poaceae & 77 \\
\hline Podagrostis & Poaceae & 1 \\
\hline Podanthus & Asteraceae & 2 \\
\hline Podocarpus & Podocarpaceae & 6 \\
\hline Podocoma & Asteraceae & 5 \\
\hline Podophorus & Poaceae & 1 \\
\hline Podostemum & Podostemaceae & 7 \\
\hline Podranea & Bignoniaceae & 1 \\
\hline Poecilanthe & Fabaceae & 1 \\
\hline Pogoniopsis & Orchidaceae & 1 \\
\hline Pogonopus & Rubiaceae & 1 \\
\hline Poikilacanthus & Acanthaceae & 1 \\
\hline Poiretia & Fabaceae & 4 \\
\hline Poissonia & Fabaceae & 2 \\
\hline Polemonium & Polemoniaceae & 1 \\
\hline Polyachyrus & Asteraceae & 7 \\
\hline Polybotrya & Dryopteridaceae & 3 \\
\hline Polycarpaea & Caryophyllaceae & 2 \\
\hline Polycarpon & Caryophyllaceae & 2 \\
\hline Polygala & Polygalaceae & 71 \\
\hline Polygonum & Polygonaceae & 28 \\
\hline Polylepis & Rosaceae & 6 \\
\hline Polyphlebium & Hymenophyllaceae & 5 \\
\hline Polypogon & Poaceae & 13 \\
\hline Polypsecadium & Brassicaceae & 9 \\
\hline Polystachya & Orchidaceae & 7 \\
\hline Polystichum & Dryopteridaceae & 13 \\
\hline Polytaenium & Pteridaceae & 4 \\
\hline Pomaria & Fabaceae & 4 \\
\hline Pombalia (**) & Violaceae & 18 \\
\hline Poncirus & Rutaceae & 1 \\
\hline Pontederia & Pontederiaceae & 8 \\
\hline Ponthieva & Orchidaceae & 3 \\
\hline Populus & Salicaceae & 3 \\
\hline Porcelia (**) & Annonaceae & 1 \\
\hline
\end{tabular}


Tabla 4. (Continuación).

\begin{tabular}{|c|c|c|}
\hline Género & Familia & $\mathbf{N}^{0} \mathrm{Sp}$. \\
\hline Porlieria & Zygophyllaceae & 2 \\
\hline Porophyllum & Asteraceae & 10 \\
\hline Portulaca & Portulacaceae & 30 \\
\hline Posoqueria & Rubiaceae & 2 \\
\hline Potamogeton & Potamogetonaceae & 10 \\
\hline Potentilla & Rosaceae & 6 \\
\hline Pourouma & Urticaceae & 1 \\
\hline Pouteria & Sapotaceae & 15 \\
\hline Pouzolzia (**) & Urticaceae & 1 \\
\hline Pozoa & Apiaceae & 2 \\
\hline Pradosia & Sapotaceae & 2 \\
\hline Praxelis (**) & Asteraceae & 7 \\
\hline Prescottia & Orchidaceae & 8 \\
\hline Presliophytum (*) & Loasaceae & 2 \\
\hline Prestonia & Apocynaceae & 8 \\
\hline Primula & Primulaceae & 3 \\
\hline Priogymnanthus & Oleaceae & 1 \\
\hline Pristimera & Celastraceae & 1 \\
\hline Priva & Verbenaceae & 1 \\
\hline Prockia & Salicaceae & 1 \\
\hline Promenaea & Orchidaceae & 4 \\
\hline Proserpinaca & Haloragaceae & 1 \\
\hline Prosopanche & Aristolochiaceae & 2 \\
\hline Prosopidastrum & Fabaceae & 6 \\
\hline Prosopis & Fabaceae & 32 \\
\hline Prosthechea & Orchidaceae & 9 \\
\hline Protium & Burseraceae & 2 \\
\hline Proustia & Asteraceae & 2 \\
\hline Prumnopitys & Podocarpaceae & 1 \\
\hline Prunella & Lamiaceae & 2 \\
\hline Prunus & Rosaceae & 20 \\
\hline Pseudabutilon & Malvaceae & 7 \\
\hline Pseudananas & Bromeliaceae & 1 \\
\hline Pseudechinolaena & Poaceae & 1 \\
\hline Pseudelephantopus & Asteraceae & 1 \\
\hline Pseuderanthemum & Acanthaceae & 2 \\
\hline Pseudobombax & Malvaceae & 6 \\
\hline Pseudocyclanthera & Cucurbitaceae & 1 \\
\hline Pseudognaphalium (**) & Asteraceae & 18 \\
\hline
\end{tabular}

\begin{tabular}{|c|c|c|}
\hline Género & Familia & $\mathbf{N}^{0}$ Sp. \\
\hline Pseudogynoxys & Asteraceae & 3 \\
\hline Pseudolmedia & Moraceae & 2 \\
\hline Pseudolycopodiella (**) & Lycopodiaceae & 3 \\
\hline Pseudopiptadenia & Fabaceae & 1 \\
\hline Pseudoplantago & Amaranthaceae & 1 \\
\hline Pseudotrimezia (**) & Iridaceae & 1 \\
\hline Pseudotsuga & Pinaceae & 1 \\
\hline Psidium & Myrtaceae & 19 \\
\hline Psiguria & Cucurbitaceae & 1 \\
\hline Psilocarphus & Asteraceae & 2 \\
\hline Psilochilus & Orchidaceae & 2 \\
\hline Psilotum & Psilotaceae & 1 \\
\hline Psittacanthus & Loranthaceae & 2 \\
\hline Psophocarpus (**) & Fabaceae & 1 \\
\hline Psoralea & Fabaceae & 1 \\
\hline Psychotria & Rubiaceae & 28 \\
\hline Pterichis & Orchidaceae & 2 \\
\hline Pteridium & Dennstaedtiaceae & 1 \\
\hline Pteris & Pteridaceae & 26 \\
\hline Pterocactus & Cactaceae & 10 \\
\hline Pterocarpus & Fabaceae & 2 \\
\hline Pterocaulon & Asteraceae & 12 \\
\hline Pteroglossa & Orchidaceae & 7 \\
\hline Pterogyne & Fabaceae & 1 \\
\hline Pterolepis & Melastomataceae & 3 \\
\hline Pteropepon & Cucurbitaceae & 1 \\
\hline Ptilochaeta & Malpighiaceae & 2 \\
\hline Puccinellia & Poaceae & 10 \\
\hline Pueraria (**) & Fabaceae & 1 \\
\hline Puna & Cactaceae & 3 \\
\hline Puya & Bromeliaceae & 24 \\
\hline Pycnophyllopsis & Caryophyllaceae & 2 \\
\hline Pycnophyllum & Caryophyllaceae & 6 \\
\hline Pyracantha & Rosaceae & 2 \\
\hline Pyrolirion & Amaryllidaceae & 1 \\
\hline Pyrostegia & Bignoniaceae & 1 \\
\hline Pyrrhocactus & Cactaceae & 27 \\
\hline Qualea & Vochysiaceae & 5 \\
\hline Quaternella & Amaranthaceae & 1 \\
\hline
\end{tabular}


Tabla 4. (Continuación).

\begin{tabular}{|c|c|c|c|c|c|}
\hline Género & Familia & $\mathbf{N}^{\circ} \mathrm{Sp}$ & Género & Familia & $\mathbf{N}^{\circ}$ Sp. \\
\hline Quechualia (**) & Asteraceae & 1 & Rhaponticum (**) & Asteraceae & 1 \\
\hline Quercus (**) & Fagaceae & 1 & Rhipidocladum & Poaceae & 2 \\
\hline Quesnelia & Bromeliaceae & 4 & Rhipsalis & Cactaceae & 24 \\
\hline Quiabentia & Cactaceae & 1 & Rhizophora & Rhizophoraceae & 1 \\
\hline Quiina & Quiinaceae & 1 & Rhodocalyx & Apocynaceae & 1 \\
\hline Quillaja & Quillajaceae & 2 & Rhodolirium (**) & Amaryllidaceae & 5 \\
\hline Quinchamalium & Schoepfiaceae & 1 & Rhodophiala & Amaryllidaceae & 13 \\
\hline Racinaea & Bromeliaceae & 2 & Rhodoscirpus (*) & Cyperaceae & 1 \\
\hline Racosperma (**) & Fabaceae & 4 & Rhodostemonodaphne & Lauraceae & 1 \\
\hline Radiovittaria & Pteridaceae & 1 & Rhynchanthera & Melastomataceae & 9 \\
\hline Radlkoferotoma (**) & Asteraceae & 3 & Rhynchoryza & Poaceae & 1 \\
\hline Ramorinoa & Fabaceae & 1 & Rhynchosia & Fabaceae & 22 \\
\hline Randia & Rubiaceae & 4 & Rhynchosida & Malvaceae & 1 \\
\hline Ranunculus & Ranunculaceae & 31 & Rhynchospora & Cyperaceae & 73 \\
\hline Raphanus & Brassicaceae & 2 & Rhytachne & Poaceae & 1 \\
\hline Rapistrum & Brassicaceae & 1 & Ribes & Grossulariaceae & 10 \\
\hline Ratibida & Asteraceae & 1 & Richardia & Rubiaceae & 8 \\
\hline Raukaua & Araliaceae & 2 & Richeria & Phyllanthaceae & 1 \\
\hline Raulinoa & Rutaceae & 1 & Ricinus & Euphorbiaceae & 1 \\
\hline Raulinoreitzia & Asteraceae & 3 & Riedeliella & Fabaceae & 1 \\
\hline Rauvolfia (**) & Apocynaceae & 4 & Rivina & Phytolaccaceae & 1 \\
\hline Rebutia & Cactaceae & 6 & Robinia & Fabaceae & 1 \\
\hline Recordia (**) & Verbenaceae & 1 & Rodriguezia & Orchidaceae & 5 \\
\hline Regnellidium & Marsileaceae & 1 & Rojasia & Apocynaceae & 1 \\
\hline Reicheella & Caryophyllaceae & 1 & Romanoa & Euphorbiaceae & 1 \\
\hline Reichenbachia & Nyctaginaceae & 1 & Romulea (**) & Iridaceae & 1 \\
\hline Reissekia (**) & Rhamnaceae & 1 & Rorippa & Brassicaceae & 12 \\
\hline Reitzia & Poaceae & 1 & Rosa & Rosaceae & 5 \\
\hline Relchela & Poaceae & 1 & Rosenbergiodendron & Rubiaceae & 1 \\
\hline Remirea & Cyperaceae & 1 & Rostkovia & Juncaceae & 1 \\
\hline Renealmia (**) & Zingiberaceae & 1 & Rostraria & Poaceae & 2 \\
\hline Reseda & Resedaceae & 5 & Rotala & Lythraceae & 2 \\
\hline Retanilla & Rhamnaceae & 4 & Rottboellia & Poaceae & 1 \\
\hline Reyesia & Solanaceae & 4 & Roupala & Proteaceae & 5 \\
\hline Rhabdadenia & Apocynaceae & 2 & Rourea & Connaraceae & 1 \\
\hline Rhabdocaulon & Lamiaceae & 6 & Rubia & Rubiaceae & 1 \\
\hline Rhamnidium & Rhamnaceae & 3 & Rubus & Rosaceae & 20 \\
\hline Rhamnus & Rhamnaceae & 3 & Rudgea & Rubiaceae & 7 \\
\hline Rhaphithamnus & Verbenaceae & 2 & Rudolfiella & Orchidaceae & 1 \\
\hline
\end{tabular}


Tabla 4. (Continuación).

\begin{tabular}{|c|c|c|}
\hline Género & Familia & $\mathbf{N}^{0}$ Sp. \\
\hline Ruehssia & Apocynaceae & 8 \\
\hline Ruellia & Acanthaceae & 27 \\
\hline Rugoloa (*) & Poaceae & 3 \\
\hline Rumex & Polygonaceae & 25 \\
\hline Rumohra & Dryopteridaceae & 3 \\
\hline Ruppia & Ruppiaceae & 3 \\
\hline Ruprechtia & Polygonaceae & 6 \\
\hline Ruta & Rutaceae & 1 \\
\hline Rytidosperma & Poaceae & 7 \\
\hline Sabicea & Rubiaceae & 1 \\
\hline Sabulina $(* *)$ & Caryophyllaceae & 2 \\
\hline Saccharum & Poaceae & 6 \\
\hline Sacciolepis & Poaceae & 2 \\
\hline Saccoloma & Saccolomataceae & 3 \\
\hline Sacoila & Orchidaceae & 5 \\
\hline Sageretia & Rhamnaceae & 1 \\
\hline Sagina & Caryophyllaceae & 4 \\
\hline Sagittaria & Alismataceae & 3 \\
\hline Salacia & Celastraceae & 3 \\
\hline Salix & Salicaceae & 6 \\
\hline Salmea & Asteraceae & 1 \\
\hline Salpichlaena & Blechnaceae & 2 \\
\hline Salpichroa & Solanaceae & 4 \\
\hline Salpiglossis & Solanaceae & 2 \\
\hline Salpinga & Melastomataceae & 1 \\
\hline Salsola & Chenopodiaceae & 2 \\
\hline Salta (*) & Polygonaceae & 1 \\
\hline Salvia & Lamiaceae & 44 \\
\hline Salvinia & Salviniaceae & 6 \\
\hline Samanea & Fabaceae & 1 \\
\hline Sambucus & Adoxaceae & 2 \\
\hline Samolus & Primulaceae & 5 \\
\hline Sanderella & Orchidaceae & 2 \\
\hline Sanguisorba & Rosaceae & 1 \\
\hline Sanicula & Apiaceae & 2 \\
\hline Sanvitalia & Asteraceae & 1 \\
\hline Sapindus & Sapindaceae & 1 \\
\hline Sapium & Euphorbiaceae & 3 \\
\hline Saponaria & Caryophyllaceae & 1 \\
\hline
\end{tabular}

\begin{tabular}{|c|c|c|}
\hline Género & Familia & $\mathbf{N}^{0}$ Sp. \\
\hline Saranthe & Marantaceae & 3 \\
\hline Sarcocornia & Chenopodiaceae & 6 \\
\hline Sarcodraba & Brassicaceae & 4 \\
\hline Sarcoglottis & Orchidaceae & 14 \\
\hline Sarcomphalus (**) & Rhamnaceae & 2 \\
\hline Sarcorhachis & Piperaceae & 1 \\
\hline Sarcotoxicum (*) & Capparaceae & 1 \\
\hline Sarmienta & Gesneriaceae & 1 \\
\hline Satureja (**) & Lamiaceae & 1 \\
\hline Sauroglossum & Orchidaceae & 4 \\
\hline Sauvagesia & Ochnaceae & 4 \\
\hline Savia & Phyllanthaceae & 1 \\
\hline Saxegothaea & Podocarpaceae & 1 \\
\hline Saxifraga & Saxifragaceae & 2 \\
\hline Saxifragella & Saxifragaceae & 1 \\
\hline Saxifragodes & Saxifragaceae & 1 \\
\hline Scabiosa & Caprifoliaceae & 1 \\
\hline Scaevola & Goodeniaceae & 1 \\
\hline Scandix & Apiaceae & 1 \\
\hline Scaphyglottis & Orchidaceae & 3 \\
\hline Sceptridium (**) & Ophioglossaceae & 3 \\
\hline Schaefferia & Celastraceae & 1 \\
\hline Schaueria & Acanthaceae & 2 \\
\hline Schedonorus & Poaceae & 1 \\
\hline Schefflera & Araliaceae & 4 \\
\hline Schenkia $(* *)$ & Gentianaceae & 1 \\
\hline Schickendantziella $(* *)$ & Amaryllidaceae & 1 \\
\hline Schindleria & Phytolaccaceae & 1 \\
\hline Schinopsis & Anacardiaceae & 7 \\
\hline Schinus & Anacardiaceae & 29 \\
\hline Schismus & Poaceae & 2 \\
\hline Schistocarpha & Asteraceae & 1 \\
\hline Schistogyne & Apocynaceae & 1 \\
\hline Schizachyrium & Poaceae & 16 \\
\hline Schizaea & Schizaeaceae & 3 \\
\hline Schizanthus & Solanaceae & 13 \\
\hline Schizolobium & Fabaceae & 1 \\
\hline Schizopetalon & Brassicaceae & 10 \\
\hline Schkuhria & Asteraceae & 1 \\
\hline
\end{tabular}


Tabla 4. (Continuación).

\begin{tabular}{|c|c|c|}
\hline Género & Familia & $\mathrm{N}^{\circ} \mathrm{Sp}$. \\
\hline Schlechtendalia & Asteraceae & 1 \\
\hline Schlegelia & Schlegeliaceae & 1 \\
\hline Schnella $(* *)$ & Fabaceae & 2 \\
\hline Schoenoplectiella (*) & Cyperaceae & 2 \\
\hline Schoenoplectus & Cyperaceae & 4 \\
\hline Schoenus & Cyperaceae & 5 \\
\hline Schoepfia & Schoepfiaceae & 2 \\
\hline Schreiteria & Montiaceae & 1 \\
\hline Schubertia & Apocynaceae & 2 \\
\hline Schultesia & Gentianaceae & 3 \\
\hline Schwartzia & Marcgraviaceae & 1 \\
\hline Schweiggeria (**) & Violaceae & 1 \\
\hline Schwenckia & Solanaceae & 5 \\
\hline Schwendenera & Rubiaceae & 1 \\
\hline Sciaphila & Triuridaceae & 1 \\
\hline Scirpus & Cyperaceae & 4 \\
\hline Scleranthus & Caryophyllaceae & 1 \\
\hline Scleria & Cyperaceae & 30 \\
\hline Sclerochloa & Poaceae & 1 \\
\hline Sclerophylax & Solanaceae & 14 \\
\hline Scleropogon & Poaceae & 1 \\
\hline Scolymus & Asteraceae & 1 \\
\hline Scoparia & Plantaginaceae & 9 \\
\hline Scorzonera & Asteraceae & 1 \\
\hline Scrophularia & Scrophulariaceae & 2 \\
\hline Scutellaria & Lamiaceae & 8 \\
\hline Scutia & Rhamnaceae & 2 \\
\hline Scuticaria & Orchidaceae & 1 \\
\hline Scybalium (**) & Balanophoraceae & 1 \\
\hline Scyphanthus & Loasaceae & 1 \\
\hline Sebastiania & Euphorbiaceae & 11 \\
\hline Secondatia & Apocynaceae & 1 \\
\hline Securidaca & Polygalaceae & 5 \\
\hline Sedum & Crassulaceae & 5 \\
\hline Seemannia (**) & Gesneriaceae & 3 \\
\hline Seguieria & Phytolaccaceae & 4 \\
\hline Selaginella & Selaginellaceae & 23 \\
\hline Selenicereus & Cactaceae & 3 \\
\hline
\end{tabular}

\begin{tabular}{|c|c|c|}
\hline Género & Familia & $\mathbf{N}^{0}$ Sp. \\
\hline Selkirkia & Boraginaceae & 3 \\
\hline Selliera & Goodeniaceae & 1 \\
\hline Sellocharis $(* *)$ & Fabaceae & 1 \\
\hline Semialarium & Celastraceae & 1 \\
\hline Senecio & Asteraceae & 415 \\
\hline Senegalia $(* *)$ & Fabaceae & 27 \\
\hline Senna & Fabaceae & 54 \\
\hline Serjania & Sapindaceae & 39 \\
\hline Serpocaulon & Polypodiaceae & 11 \\
\hline Sesbania & Fabaceae & 6 \\
\hline Seseli & Apiaceae & 1 \\
\hline Sessea & Solanaceae & 2 \\
\hline Sesuvium & Aizoaceae & 1 \\
\hline Setaria & Poaceae & 37 \\
\hline Setiechinopsis & Cactaceae & 1 \\
\hline Sherardia & Rubiaceae & 1 \\
\hline Sibara (**) & Brassicaceae & 6 \\
\hline Sibthorpia & Plantaginaceae & 2 \\
\hline Sicydium & Cucurbitaceae & 2 \\
\hline Sicyos & Cucurbitaceae & 8 \\
\hline Sida & Malvaceae & 76 \\
\hline Sidastrum & Malvaceae & 3 \\
\hline Sideroxylon & Sapotaceae & 1 \\
\hline Sigesbeckia & Asteraceae & 2 \\
\hline Sigmoidotropis (*) & Fabaceae & 1 \\
\hline Silene & Caryophyllaceae & 25 \\
\hline Silybum & Asteraceae & 1 \\
\hline Simaba & Simaroubaceae & 1 \\
\hline Simira & Rubiaceae & 3 \\
\hline Simsia & Asteraceae & 1 \\
\hline Sinapis & Brassicaceae & 2 \\
\hline Sinningia & Gesneriaceae & 29 \\
\hline Siolmatra & Cucurbitaceae & 1 \\
\hline Sipanea & Rubiaceae & 1 \\
\hline Siparuna & Siparunaceae & 2 \\
\hline Siphanthera (**) & Melastomataceae & 1 \\
\hline Siphocampylus & Campanulaceae & 23 \\
\hline Siphoneugena & Myrtaceae & 4 \\
\hline
\end{tabular}


Tabla 4. (Continuación).

\begin{tabular}{|c|c|c|}
\hline Género & Familia & $\mathbf{N}^{0}$ Sp. \\
\hline Sisymbrium & Brassicaceae & 7 \\
\hline Sisyrinchium & Iridaceae & 97 \\
\hline Sium & Apiaceae & 1 \\
\hline Skeptrostachys & Orchidaceae & 10 \\
\hline Skytanthus & Apocynaceae & 1 \\
\hline Sloanea & Elaeocarpaceae & 5 \\
\hline Smallanthus & Asteraceae & 5 \\
\hline Smilax & Smilacaceae & 14 \\
\hline Solanum & Solanaceae & 216 \\
\hline Solaria & Amaryllidaceae & 2 \\
\hline Soleirolia & Urticaceae & 1 \\
\hline Solenomelus & Iridaceae & 2 \\
\hline Solidago & Asteraceae & 5 \\
\hline Soliva & Asteraceae & 5 \\
\hline Sommerfeltia & Asteraceae & 2 \\
\hline Sonchus & Asteraceae & 15 \\
\hline Sophora & Fabaceae & 7 \\
\hline Sorbus & Rosaceae & 1 \\
\hline Sorghastrum & Poaceae & 8 \\
\hline Sorghum & Poaceae & 3 \\
\hline Sorocea & Moraceae & 4 \\
\hline Sparattosperma & Bignoniaceae & 1 \\
\hline Sparaxis & Iridaceae & 2 \\
\hline Spartium & Fabaceae & 1 \\
\hline Spathantheum & Araceae & 1 \\
\hline Spathicarpa & Araceae & 2 \\
\hline Specklinia & Orchidaceae & 5 \\
\hline Speea & Amaryllidaceae & 1 \\
\hline Spegazziniophytum (**) & Euphorbiaceae & 1 \\
\hline Spergula & Caryophyllaceae & 14 \\
\hline Spergularia & Caryophyllaceae & 15 \\
\hline Spermacoce & Rubiaceae & 13 \\
\hline Spermolepis & Apiaceae & 1 \\
\hline Sphaeralcea & Malvaceae & 14 \\
\hline Sphaerophysa & Fabaceae & 1 \\
\hline Sphagneticola & Asteraceae & 2 \\
\hline Sphenoclea & Sphenocleaceae & 1 \\
\hline Sphinctanthus & Rubiaceae & 1 \\
\hline
\end{tabular}

\begin{tabular}{|c|c|c|}
\hline Género & Familia & $\mathbf{N}^{0}$ Sp. \\
\hline Spigelia & Loganiaceae & 23 \\
\hline Spilanthes & Asteraceae & 4 \\
\hline Spinoliva (*) & Asteraceae & 1 \\
\hline Spiraea & Rosaceae & 2 \\
\hline Spirodela & Araceae & 1 \\
\hline Spirotheca & Malvaceae & 1 \\
\hline Sporobolus & Poaceae & 22 \\
\hline Stachycephalum & Asteraceae & 1 \\
\hline Stachys & Lamiaceae & 12 \\
\hline Stachytarpheta & Verbenaceae & 8 \\
\hline Staelia & Rubiaceae & 4 \\
\hline Stangea & Caprifoliaceae & 1 \\
\hline Stanhopea & Orchidaceae & 3 \\
\hline Stapfochloa (*) & Poaceae & 6 \\
\hline Staurogyne & Acanthaceae & 4 \\
\hline Steinchisma & Poaceae & 4 \\
\hline Steiropteris (**) & Thelypteridaceae & 4 \\
\hline Stelis & Orchidaceae & 15 \\
\hline Stellaria & Caryophyllaceae & 17 \\
\hline Stemodia & Plantaginaceae & 13 \\
\hline Stenachaenium & Asteraceae & 5 \\
\hline Stenandrium & Acanthaceae & 6 \\
\hline Stenocephalum (**) & Asteraceae & 4 \\
\hline Stenodraba $(* *)$ & Brassicaceae & 8 \\
\hline Stenodrepanum & Fabaceae & 1 \\
\hline Stenogrammitis (*) & Polypodiaceae & 1 \\
\hline Stenotaphrum & Poaceae & 1 \\
\hline Stephostachys (*) & Poaceae & 1 \\
\hline Sterculia & Malvaceae & 1 \\
\hline Stetsonia & Cactaceae & 1 \\
\hline Stevia & Asteraceae & 66 \\
\hline Steyermarkia (**) & Asteraceae & 3 \\
\hline Sticherus & Gleicheniaceae & 13 \\
\hline Stifftia $(* *)$ & Asteraceae & 1 \\
\hline Stigmaphyllon & Malpighiaceae & 6 \\
\hline Stigmatopteris & Dryopteridaceae & 5 \\
\hline Stigmatosema & Orchidaceae & 3 \\
\hline Stillingia & Euphorbiaceae & 5 \\
\hline
\end{tabular}


Tabla 4. (Continuación).

\begin{tabular}{|c|c|c|}
\hline Género & Familia & $\mathbf{N}^{0}$ Sp. \\
\hline Stizophyllum & Bignoniaceae & 1 \\
\hline Stomatanthes $(* *)$ & Asteraceae & 2 \\
\hline Streptochaeta & Poaceae & 1 \\
\hline Strigosella & Brassicaceae & 1 \\
\hline Stromanthe & Marantaceae & 5 \\
\hline Strophopappus (**) & Asteraceae & 1 \\
\hline Struchium & Asteraceae & 1 \\
\hline Struthanthus & Loranthaceae & 5 \\
\hline Strychnos & Loganiaceae & 7 \\
\hline Stryphnodendron & Fabaceae & 2 \\
\hline Stuckenia & Potamogetonaceae & 3 \\
\hline Stylogyne $(* *)$ & Primulaceae & 1 \\
\hline Stylosanthes & Fabaceae & 16 \\
\hline Styrax & Styracaceae & 9 \\
\hline Suaeda & Chenopodiaceae & 10 \\
\hline Swartzia & Fabaceae & 3 \\
\hline Sweetia & Fabaceae & 2 \\
\hline Syagrus & Arecaceae & 8 \\
\hline Symphoricarpos & Caprifoliaceae & 1 \\
\hline Symphyopappus (**) & Asteraceae & 6 \\
\hline Symphyotrichum & Asteraceae & 8 \\
\hline Symphytum & Boraginaceae & 1 \\
\hline Symplocos & Symplocaceae & 21 \\
\hline Synammia & Polypodiaceae & 3 \\
\hline Synandrospadix & Araceae & 1 \\
\hline Synedrella & Asteraceae & 1 \\
\hline Synedrellopsis & Asteraceae & 1 \\
\hline Syngonanthus & Eriocaulaceae & 7 \\
\hline Syngonium (**) & Araceae & 2 \\
\hline Syringa & Oleaceae & 1 \\
\hline Syzygium (**) & Myrtaceae & 2 \\
\hline Tabebuia & Bignoniaceae & 4 \\
\hline Tabernaemontana & Apocynaceae & 1 \\
\hline Taccarum & Araceae & 2 \\
\hline Tachigali & Fabaceae & 3 \\
\hline Taeniatherum & Poaceae & 1 \\
\hline Tagetes & Asteraceae & 14 \\
\hline Talinum & Talinaceae & 5 \\
\hline
\end{tabular}

\begin{tabular}{|c|c|c|}
\hline Género & Familia & $\mathbf{N}^{0}$ Sp. \\
\hline Talipariti & Malvaceae & 1 \\
\hline Talisia & Sapindaceae & 2 \\
\hline Tamarix & Tamaricaceae & 1 \\
\hline Tamonea & Verbenaceae & 1 \\
\hline Tanacetum & Asteraceae & 3 \\
\hline Tanaecium & Bignoniaceae & 5 \\
\hline Tapeinia & Iridaceae & 1 \\
\hline Tapirira & Anacardiaceae & 1 \\
\hline $\operatorname{Tara}(* *)$ & Fabaceae & 1 \\
\hline Tarasa & Malvaceae & 18 \\
\hline Taraxacum & Asteraceae & 12 \\
\hline Tarenaya (**) & Cleomaceae & 14 \\
\hline Tassadia & Apocynaceae & 2 \\
\hline Tecoma & Bignoniaceae & 4 \\
\hline Tecophilaea & Tecophilaeaceae & 2 \\
\hline Tectaria & Tectariaceae & 3 \\
\hline Teesdalia & Brassicaceae & 1 \\
\hline Telmatoblechnum (*) & Blechnaceae & 1 \\
\hline Temnadenia & Apocynaceae & 2 \\
\hline Tephrocactus & Cactaceae & 7 \\
\hline Tephrosia & Fabaceae & 6 \\
\hline Teramnus $(* *)$ & Fabaceae & 1 \\
\hline Terminalia & Combretaceae & 5 \\
\hline Ternstroemia & Pentaphylacaceae & 2 \\
\hline Terpsichore & Polypodiaceae & 1 \\
\hline Tessaria & Asteraceae & 5 \\
\hline Tetilla & Francoaceae & 1 \\
\hline Tetracera & Dilleniaceae & 4 \\
\hline Tetrachondra & Tetrachondraceae & 1 \\
\hline Tetraglochin & Rosaceae & 6 \\
\hline Tetragonia & Aizoaceae & 10 \\
\hline Tetrapanax (**) & Araliaceae & 1 \\
\hline Tetrapollinia (**) & Gentianaceae & 1 \\
\hline Tetrapterys & Malpighiaceae & 9 \\
\hline Tetrastylidum (**) & Olacaceae & 1 \\
\hline Tetroncium & Juncaginaceae & 1 \\
\hline Tetrorchidium & Euphorbiaceae & 2 \\
\hline Teucrium & Lamiaceae & 5 \\
\hline
\end{tabular}


Tabla 4. (Continuación).

\begin{tabular}{|c|c|c|}
\hline Género & Familia & $\mathbf{N}^{0} \mathrm{Sp}$. \\
\hline Thalia & Marantaceae & 2 \\
\hline Thalictrum & Ranunculaceae & 2 \\
\hline Thamnoseris & Asteraceae & 1 \\
\hline Thaumatocaryon & Boraginaceae & 2 \\
\hline Thaumatophyllum (**) & Araceae & 6 \\
\hline Thelesperma & Asteraceae & 1 \\
\hline Thelocephala & Cactaceae & 7 \\
\hline Thelypteris & Thelypteridaceae & 8 \\
\hline Themeda & Poaceae & 1 \\
\hline Thesium & Thesiaceae & 2 \\
\hline Thespesia (**) & Malvaceae & 1 \\
\hline Thevetia & Apocynaceae & 2 \\
\hline Thinopyrum & Poaceae & 1 \\
\hline Thinouia & Sapindaceae & 4 \\
\hline Thismia (**) & Thismiaceae & 1 \\
\hline Thlaspi & Brassicaceae & 1 \\
\hline Thryallis & Malpighiaceae & 2 \\
\hline Thunbergia & Acanthaceae & 3 \\
\hline Thymophylla & Asteraceae & 1 \\
\hline Thyrsacanthus (**) & Acanthaceae & 2 \\
\hline Thyrsopteris & Dicksoniaceae & 1 \\
\hline Tibouchina & Melastomataceae & 47 \\
\hline Tigridia & Iridaceae & 1 \\
\hline Tilesia & Asteraceae & 1 \\
\hline Tillandsia & Bromeliaceae & 91 \\
\hline Tinantia & Commelinaceae & 1 \\
\hline Tipuana & Fabaceae & 1 \\
\hline Tiquilia & Boraginaceae & 6 \\
\hline Tithonia & Asteraceae & 3 \\
\hline Tocoyena & Rubiaceae & 2 \\
\hline Tolpis & Asteraceae & 1 \\
\hline Tomostima (**) & Brassicaceae & 1 \\
\hline Tontelea & Celastraceae & 2 \\
\hline Topea (*) & Apocynaceae & 2 \\
\hline Tordylium & Apiaceae & 1 \\
\hline Torenia (**) & Linderniaceae & 1 \\
\hline Torilis & Apiaceae & 2 \\
\hline Toulicia & Sapindaceae & 1 \\
\hline
\end{tabular}

\begin{tabular}{|c|c|c|}
\hline Género & Familia & $\mathbf{N}^{\circ}$ Sp. \\
\hline Tournefortia & Boraginaceae & 4 \\
\hline Tourrettia & Bignoniaceae & 1 \\
\hline Tovomitopsis $(* *)$ & Clusiaceae & 1 \\
\hline Trachycarpus & Arecaceae & 1 \\
\hline Trachypogon & Poaceae & 2 \\
\hline Trachypteris & Pteridaceae & 1 \\
\hline Tradescantia & Commelinaceae & 20 \\
\hline Tragia & Euphorbiaceae & 16 \\
\hline Tragopogon & Asteraceae & 3 \\
\hline Tragus & Poaceae & 4 \\
\hline Traubia & Amaryllidaceae & 1 \\
\hline Trema & Cannabaceae & 1 \\
\hline Trembleya & Melastomataceae & 2 \\
\hline Tressenia (*) & Apocynaceae & 1 \\
\hline Trevoa & Rhamnaceae & 1 \\
\hline Trianthema & Aizoaceae & 2 \\
\hline Tribeles & Escalloniaceae & 1 \\
\hline Tribulus & Zygophyllaceae & 1 \\
\hline Trichanthecium (*) & Poaceae & 6 \\
\hline Trichilia & Meliaceae & 12 \\
\hline Trichocentrum & Orchidaceae & 3 \\
\hline Trichocereus & Cactaceae & 30 \\
\hline Trichocline & Asteraceae & 21 \\
\hline Trichogonia & Asteraceae & 5 \\
\hline Trichogoniopsis (**) & Asteraceae & 1 \\
\hline Trichomanes & Hymenophyllaceae & 11 \\
\hline Trichoneura & Poaceae & 1 \\
\hline Trichopetalum & Asparagaceae & 2 \\
\hline Trichophorum & Cyperaceae & 1 \\
\hline Trichosalpinx & Orchidaceae & 2 \\
\hline Trichostigma & Phytolaccaceae & 1 \\
\hline Trichotolinum & Brassicaceae & 1 \\
\hline Tricomaria & Malpighiaceae & 1 \\
\hline Tridax & Asteraceae & 2 \\
\hline Tridens & Poaceae & 4 \\
\hline Trifolium & Fabaceae & 34 \\
\hline Triglochin & Juncaginaceae & 5 \\
\hline Trigonella & Fabaceae & 1 \\
\hline
\end{tabular}


Tabla 4. (Continuación).

\begin{tabular}{|c|c|c|}
\hline Género & Familia & $\mathbf{N}^{0} \mathrm{Sp}$. \\
\hline Trigonia & Trigoniaceae & 2 \\
\hline Trigonidium & Orchidaceae & 3 \\
\hline Trihesperus (**) & Asparagaceae & 1 \\
\hline Trimezia & Iridaceae & 2 \\
\hline Triodanis & Campanulaceae & 1 \\
\hline Triphora & Orchidaceae & 2 \\
\hline Triplaris & Polygonaceae & 2 \\
\hline Tripleurospermum & Asteraceae & 1 \\
\hline Tripodanthus & Loranthaceae & 2 \\
\hline Tripogandra & Commelinaceae & 4 \\
\hline Tripogon & Poaceae & 2 \\
\hline Tripogonella (*) & Poaceae & 1 \\
\hline Tripsacum & Poaceae & 1 \\
\hline Triptilion & Asteraceae & 7 \\
\hline Trisetum & Poaceae & 13 \\
\hline Tristachya & Poaceae & 1 \\
\hline Tristagma & Amaryllidaceae & 14 \\
\hline Tristerix & Loranthaceae & 3 \\
\hline Tristicha & Podostemaceae & 1 \\
\hline Trithrinax & Arecaceae & 3 \\
\hline Triumfetta & Malvaceae & 5 \\
\hline Trixis & Asteraceae & 13 \\
\hline Trizeuxis & Orchidaceae & 1 \\
\hline Tropaeolum & Tropaeolaceae & 34 \\
\hline Tropidocarpum & Brassicaceae & 1 \\
\hline Tropidococcus & Malvaceae & 1 \\
\hline Tryonia (*) & Pteridaceae & 2 \\
\hline Tunilla & Cactaceae & 3 \\
\hline Turbina & Convolvulaceae & 2 \\
\hline Turnera & Turneraceae & 19 \\
\hline Tweedia & Apocynaceae & 6 \\
\hline Tynanthus & Bignoniaceae & 4 \\
\hline Typha & Typhaceae & 4 \\
\hline Typhonium (**) & Araceae & 1 \\
\hline Ugni & Myrtaceae & 3 \\
\hline Uleiorchis & Orchidaceae & 2 \\
\hline Ulex & Fabaceae & 1 \\
\hline Ullucus & Basellaceae & 1 \\
\hline
\end{tabular}

\begin{tabular}{|c|c|c|}
\hline Género & Familia & $\mathbf{N}^{0}$ Sp. \\
\hline Ulmus (**) & Ulmaceae & 1 \\
\hline Unonopsis (**) & Annonaceae & 1 \\
\hline Urena & Malvaceae & 1 \\
\hline Urera & Urticaceae & 5 \\
\hline Urmenetea & Asteraceae & 1 \\
\hline Urocarpidium & Malvaceae & 8 \\
\hline Urochloa & Poaceae & 15 \\
\hline Urolepis (**) & Asteraceae & 1 \\
\hline Urospatha (**) & Araceae & 1 \\
\hline Urospermum & Asteraceae & 1 \\
\hline Urtica & Urticaceae & 14 \\
\hline Urvillea & Sapindaceae & 8 \\
\hline Utricularia & Lentibulariaceae & 22 \\
\hline Vaccaria & Caryophyllaceae & 1 \\
\hline Vaccinium & Ericaceae & 1 \\
\hline Vachellia (**) & Fabaceae & 10 \\
\hline Vahlodea & Poaceae & 1 \\
\hline Valdivia & Escalloniaceae & 1 \\
\hline Valeriana & Caprifoliaceae & 84 \\
\hline Valerianella & Caprifoliaceae & 4 \\
\hline Vallea & Elaeocarpaceae & 1 \\
\hline Vallesia & Apocynaceae & 1 \\
\hline Vandenboschia $(* *)$ & Hymenophyllaceae & 3 \\
\hline Vanilla & Orchidaceae & 9 \\
\hline Vantanea & Humiriaceae & 1 \\
\hline Varronia & Boraginaceae & 10 \\
\hline Vasconcellea $(* *)$ & Caricaceae & 2 \\
\hline Vassobia & Solanaceae & 1 \\
\hline Vatairea (**) & Fabaceae & 1 \\
\hline Velloziella & Orobanchaceae & 1 \\
\hline Verbascum & Scrophulariaceae & 4 \\
\hline Verbena & Verbenaceae & 18 \\
\hline Verbesina & Asteraceae & 13 \\
\hline Vernicia $(* *)$ & Euphorbiaceae & 1 \\
\hline Vernonanthura (**) & Asteraceae & 40 \\
\hline Vernonia & Asteraceae & 4 \\
\hline Veronica & Plantaginaceae & 11 \\
\hline Vestia & Solanaceae & 1 \\
\hline
\end{tabular}


Tabla 4. (Continuación).

\begin{tabular}{|c|c|c|}
\hline Género & Familia & $\mathrm{N}^{\circ} \mathrm{Sp}$. \\
\hline Veyretia & Orchidaceae & 4 \\
\hline Viburnum & Adoxaceae & 1 \\
\hline Vicia & Fabaceae & 37 \\
\hline Victoria & Nymphaeaceae & 1 \\
\hline Vigna & Fabaceae & 5 \\
\hline Viguiera & Asteraceae & 1 \\
\hline Villanova & Asteraceae & 2 \\
\hline Vinca & Apocynaceae & 1 \\
\hline Viola & Violaceae & 109 \\
\hline Virola & Myristicaceae & 1 \\
\hline Vitex & Lamiaceae & 7 \\
\hline Vitis & Vitaceae & 1 \\
\hline Vittaria & Pteridaceae & 4 \\
\hline Vittetia (**) & Asteraceae & 1 \\
\hline Viviania & Francoaceae & 4 \\
\hline Vochysia & Vochysiaceae & 4 \\
\hline Volutaria (**) & Asteraceae & 1 \\
\hline Voyria & Gentianaceae & 2 \\
\hline Vriesea & Bromeliaceae & 45 \\
\hline Wahlenbergia & Campanulaceae & 9 \\
\hline Waltheria & Malvaceae & 10 \\
\hline Warmingia & Orchidaceae & 1 \\
\hline Warrea & Orchidaceae & 1 \\
\hline Watsonia & Iridaceae & 1 \\
\hline Weberbauera & Brassicaceae & 4 \\
\hline Weberbauerella (**) & Fabaceae & 1 \\
\hline Wedelia & Asteraceae & 7 \\
\hline Weingartia & Cactaceae & 1 \\
\hline Weinmannia & Cunoniaceae & 7 \\
\hline Werneria & Asteraceae & 10 \\
\hline Wettsteiniola & Podostemaceae & 2 \\
\hline Wigginsia & Cactaceae & 7 \\
\hline Wilbrandia & Cucurbitaceae & 4 \\
\hline Willkommia & Poaceae & 1 \\
\hline Wissadula & Malvaceae & 16 \\
\hline Wittrockia & Bromeliaceae & 2 \\
\hline Wolffia & Araceae & 2 \\
\hline Wolffiella & Araceae & 2 \\
\hline
\end{tabular}

\begin{tabular}{|c|c|c|}
\hline Género & Familia & $\mathbf{N}^{\circ} \mathbf{S p}$. \\
\hline Woodsia & Woodsiaceae & 1 \\
\hline Wullschlaegelia & Orchidaceae & 1 \\
\hline Xanthium & Asteraceae & 4 \\
\hline Xanthosoma & Araceae & 5 \\
\hline Xenophyllum & Asteraceae & 6 \\
\hline Xerochrysum (**) & Asteraceae & 1 \\
\hline Xerodraba & Brassicaceae & 5 \\
\hline Ximenia & Olacaceae & 2 \\
\hline Xylobium & Orchidaceae & 2 \\
\hline Xylophragma & Bignoniaceae & 2 \\
\hline Xylopia & Annonaceae & 4 \\
\hline Xylosma & Salicaceae & 9 \\
\hline Xyris & Xyridaceae & 31 \\
\hline Yavia & Cactaceae & 1 \\
\hline Youngia (**) & Asteraceae & 1 \\
\hline Yисса & Asparagaceae & 2 \\
\hline Yunquea & Asteraceae & 1 \\
\hline Zameioscirpus & Cyperaceae & 3 \\
\hline Zannichellia & Potamogetonaceae & 1 \\
\hline Zantedeschia & Araceae & 1 \\
\hline Zanthoxylum & Rutaceae & 13 \\
\hline Zapoteca & Fabaceae & 2 \\
\hline Zephyra & Tecophilaeaceae & 2 \\
\hline Zephyranthes & Amaryllidaceae & 20 \\
\hline Zeuxine (**) & Orchidaceae & 1 \\
\hline Zeyheria & Bignoniaceae & 2 \\
\hline Zinnia & Asteraceae & 1 \\
\hline Zizaniopsis & Poaceae & 4 \\
\hline Zollernia & Fabaceae & 2 \\
\hline Zootrophion & Orchidaceae & 1 \\
\hline Zornia & Fabaceae & 15 \\
\hline Zostera (**) & Zosteraceae & 1 \\
\hline Zuccagnia & Fabaceae & 1 \\
\hline Zuloagocardamum (*) & Brassicaceae & 1 \\
\hline Zygia & Fabaceae & 4 \\
\hline Zygopetalum & Orchidaceae & 4 \\
\hline Zygophlebia & Polypodiaceae & 1 \\
\hline Zygostates & Orchidaceae & 8 \\
\hline Zygostigma & Gentianaceae & 1 \\
\hline
\end{tabular}


A nivel de especie y categorías infraespecíficas han habido innumerables cambios, debidos a la descripción de nuevos taxones, nuevas citas para el área, nuevas combinaciones y sinonimias propuestas; también a la revalidación de taxones o al esclarecimiento de otros tantos que figuraban como dudosos o cuya presencia en la región del Cono Sur no era completamente certera. El catálogo en su versión actual registra 18931 especies, de las cuales 7713 son endémicas, 9892 nativas y 1326 introducidas. Al comparar estos resultados con la versión original del catálogo surge que el número de especies se ha incrementado en 1238 , lo que representa un incremento cercano al 7\%; a su vez, se contabilizaron 22 especies endémicas menos. En este punto cabe aclarar que Ulloa et al. (2017), al analizar las plantas nativas del continente americano, citaron 13125 especies para el Cono Sur, frente a las 17605 citadas en la presente contribución (este número se obtiene de la suma de especies endémicas y nativas). Esta notable diferencia puede explicarse por: 1) Ulloa et al. (2017) no consideraron a los estados del sur de Brasil (Paraná, Santa Catarina y Rio Grande do Sul) dentro del área del Cono Sur, sino dentro del área total de Brasil; 2) desde la publicación del trabajo de Ulloa et al. (2017) han surgido novedades florísticas para el área.

\section{Endemismo}

Las familias endémicas del Cono Sur son 5: Aextoxicaceae (Argentina y Chile), Gomortegaceae (Chile). Halophytaceae (Argentina), Misodendraceae (Argentina y Chile) y Philesiaceae (Argentina y Chile).

En cuantoa los géneros, para elCono Sur se registran 248 géneros endémicos (Tabla 5), pertenecientes a 66 familias; de éstos, 154 son monotípicos. Del total de géneros endémicos, 159 habitan en la Argentina, de los cuales 48 son exclusivos de este país; 2 se registran en el Sur de Brasil; 168 en Chile (73 exclusivos); 8 en Paraguay (1 exclusivo: Bordasia, Malvaceae); y 20 en Uruguay (1 exclusivo: Heterotristicha, Podostemaceae). Un total de 88 géneros endémicos son comunes a la Argentina y Chile.

Del total de especies registradas para el Cono Sur (18931) 7713 especies son endémicas, ca. 41\% del total (Tablas 1 y 3 ), un número muy significativo para áreas templadas a templado-frías.

Tabla 5. Géneros endémicos del Cono Sur, número de especies y distribución por países.

\begin{tabular}{|c|c|c|c|c|c|c|}
\hline Familia & Género & No'sp. & & & Países & \\
\hline Aextoxicaceae & Aextoxicon & 1 & Argentina & & Chile & \\
\hline Amaranthaceae & Quaternella & 1 & & S Brasil & & \\
\hline Amaryllidaceae & Beauverdia & 4 & Argentina & S Brasil & & Uruguay \\
\hline & Eithea & 1 & & S Brasil & & \\
\hline & Famatina & 2 & Argentina & & Chile & \\
\hline & Gethyum & 2 & & & Chile & \\
\hline & Gilliesia & 4 & Argentina & & Chile & \\
\hline & Ipheion & 4 & Argentina & & Chile & Uruguay \\
\hline & Latace & 2 & Argentina & & Chile & \\
\hline & Leucocoryne & 46 & & & Chile & \\
\hline & Miersia & 4 & & & Chile & \\
\hline & Phycella & 6 & Argentina & & Chile & \\
\hline & Placea & 5 & & & Chile & \\
\hline & Solaria & 2 & Argentina & & Chile & \\
\hline & Speea & 1 & & & Chile & \\
\hline
\end{tabular}


Tabla 5. (Continuación).

\begin{tabular}{|c|c|c|c|c|c|c|c|}
\hline \multirow[t]{2}{*}{ Familia } & \multirow{2}{*}{$\begin{array}{l}\text { Género } \\
\text { Traubia }\end{array}$} & \multirow{2}{*}{$\begin{array}{c}\text { No'sp. } \\
1\end{array}$} & \multicolumn{5}{|c|}{ Países } \\
\hline & & & & & Chile & & \\
\hline & Tristagma & 14 & Argentina & & Chile & & \\
\hline \multirow[t]{9}{*}{ Apiaceae } & Asteriscium & 9 & Argentina & & Chile & & \\
\hline & Austropeucedanum & 1 & Argentina & & & & \\
\hline & Bolax & 2 & Argentina & & Chile & & \\
\hline & Diposis & 3 & Argentina & & Chile & & Uruguay \\
\hline & Gymnophyton & 6 & Argentina & & Chile & & \\
\hline & Homalocarpus & 6 & & & Chile & & \\
\hline & Notiosciadium & 1 & Argentina & & & & Uruguay \\
\hline & Oligocladus & 1 & Argentina & & & & \\
\hline & Pozoa & 2 & Argentina & & Chile & & \\
\hline \multirow[t]{5}{*}{ Apocynaceae } & Brargentina & 1 & Argentina & S Brasil & & Paraguay & \\
\hline & Diplolepis & 14 & Argentina & & Chile & & \\
\hline & Elytropus & 1 & & & Chile & & \\
\hline & Topea & 1 & Argentina & & & Paraguay & \\
\hline & Tressenia & 1 & Argentina & & & & \\
\hline Araceae & Mangonia & 2 & & S Brasil & & & Uruguay \\
\hline \multirow[t]{2}{*}{ Arecaceae } & Juania & 1 & & & Chile & & \\
\hline & Jubaea & 1 & & & Chile & & \\
\hline Aristolochiaceae & Lactoris & 1 & & & Chile & & \\
\hline \multirow[t]{2}{*}{ Asparagaceae } & Clara & 3 & Argentina & S Brasil & & Paraguay & \\
\hline & Trichopetalum & 2 & Argentina & & Chile & & \\
\hline \multirow[t]{17}{*}{ Asteraceae } & Acrisione & 1 & Argentina & & Chile & & \\
\hline & Ameghinoa & 1 & Argentina & & & & \\
\hline & Asteropsis & 1 & & S Brasil & & & Uruguay \\
\hline & Aylacophora & 1 & Argentina & & & & \\
\hline & Brachyclados & 3 & Argentina & & Chile & & \\
\hline & Burkartia & 1 & Argentina & & & & \\
\hline & Cabreraea & 1 & Argentina & & & & \\
\hline & Calopappus & 1 & & & Chile & & \\
\hline & Centaurodendron & 2 & & & Chile & & \\
\hline & Chiliophyllum & 1 & Argentina & & & & \\
\hline & Chiliotrichum & 2 & Argentina & & Chile & & \\
\hline & Criscia & 1 & Argentina & S Brasil & & & Uruguay \\
\hline & Cyclolepis & 1 & Argentina & & & Paraguay & \\
\hline & Dolichlasium & 1 & Argentina & & & & \\
\hline & Doniophyton & 2 & Argentina & & Chile & & \\
\hline & Duseniella & 1 & Argentina & & & & \\
\hline & Eriachaenium & 1 & Argentina & & & & \\
\hline
\end{tabular}


Tabla 5. (Continuación).

\begin{tabular}{|c|c|c|c|c|c|c|}
\hline \multirow[t]{2}{*}{ Familia } & \multirow{2}{*}{\begin{tabular}{|c|} 
Género \\
Famatinanthus \\
\end{tabular}} & \multirow{2}{*}{$\begin{array}{c}\mathbf{N}^{\mathbf{0}} \mathbf{S p} . \\
1\end{array}$} & \multicolumn{4}{|c|}{ Países } \\
\hline & & & Argentina & & & \\
\hline & Guynesomia & 1 & & & Chile & \\
\hline & Gypothamnium & 1 & & & Chile & \\
\hline & Haroldia & 1 & Argentina & & & \\
\hline & Heterothalamulopsis & 1 & & S Brasil & & \\
\hline & Huarpea & 1 & Argentina & & & \\
\hline & Ianthopappus & 1 & Argentina & S Brasil & & Uruguay \\
\hline & Katinasia & 1 & Argentina & & & \\
\hline & Kieslingia & 1 & & & Chile & \\
\hline & Lepidophyllum & 1 & Argentina & & Chile & \\
\hline & Leptinella & 1 & Argentina & & Chile & \\
\hline & Leptocarpha & 1 & & & Chile & \\
\hline & Leunisia & 1 & & & Chile & \\
\hline & Lycapsus & 1 & & & Chile & \\
\hline & Macrachaenium & 1 & Argentina & & & \\
\hline & Marticorenia & 1 & & & Chile & \\
\hline & Microgyne & 1 & Argentina & S Brasil & & Uruguay \\
\hline & Micropsis & 5 & Argentina & & Chile & Uruguay \\
\hline & Moscharia & 2 & & & Chile & \\
\hline & Nardophyllum & 5 & Argentina & & Chile & \\
\hline & Oxyphyllum & 1 & & & Chile & \\
\hline & Panphalea & 9 & Argentina & S Brasil & & Uruguay \\
\hline & Pleocarphus & 1 & & & Chile & \\
\hline & Podanthus & 2 & & & Chile & \\
\hline & Radlkoferotoma & 3 & & S Brasil & & Uruguay \\
\hline & Sommerfeltia & 2 & Argentina & S Brasil & & Uruguay \\
\hline & Spinoliva & 1 & & & Chile & \\
\hline & Thamnoseris & 1 & & & Chile & \\
\hline & Triptilion & 7 & Argentina & & Chile & \\
\hline & Urmenetea & 1 & Argentina & & Chile & \\
\hline & Yunquea & 1 & & & Chile & \\
\hline Atherospermataceae & Laureliopsis & 1 & Argentina & & Chile & \\
\hline Bignoniaceae & Campsidium & 1 & Argentina & & Chile & \\
\hline \multirow[t]{2}{*}{ Boraginaceae } & Ixorhea & 1 & Argentina & & & \\
\hline & Nesocaryum & 1 & & & Chile & \\
\hline \multirow[t]{4}{*}{ Brassicaceae } & Aimara & 1 & & & Chile & \\
\hline & Atacama & 1 & & & Chile & \\
\hline & Alshebazia & 1 & Argentina & & Chile & \\
\hline & Chilocardamum & 4 & Argentina & & & \\
\hline
\end{tabular}


Tabla 5. (Continuación).

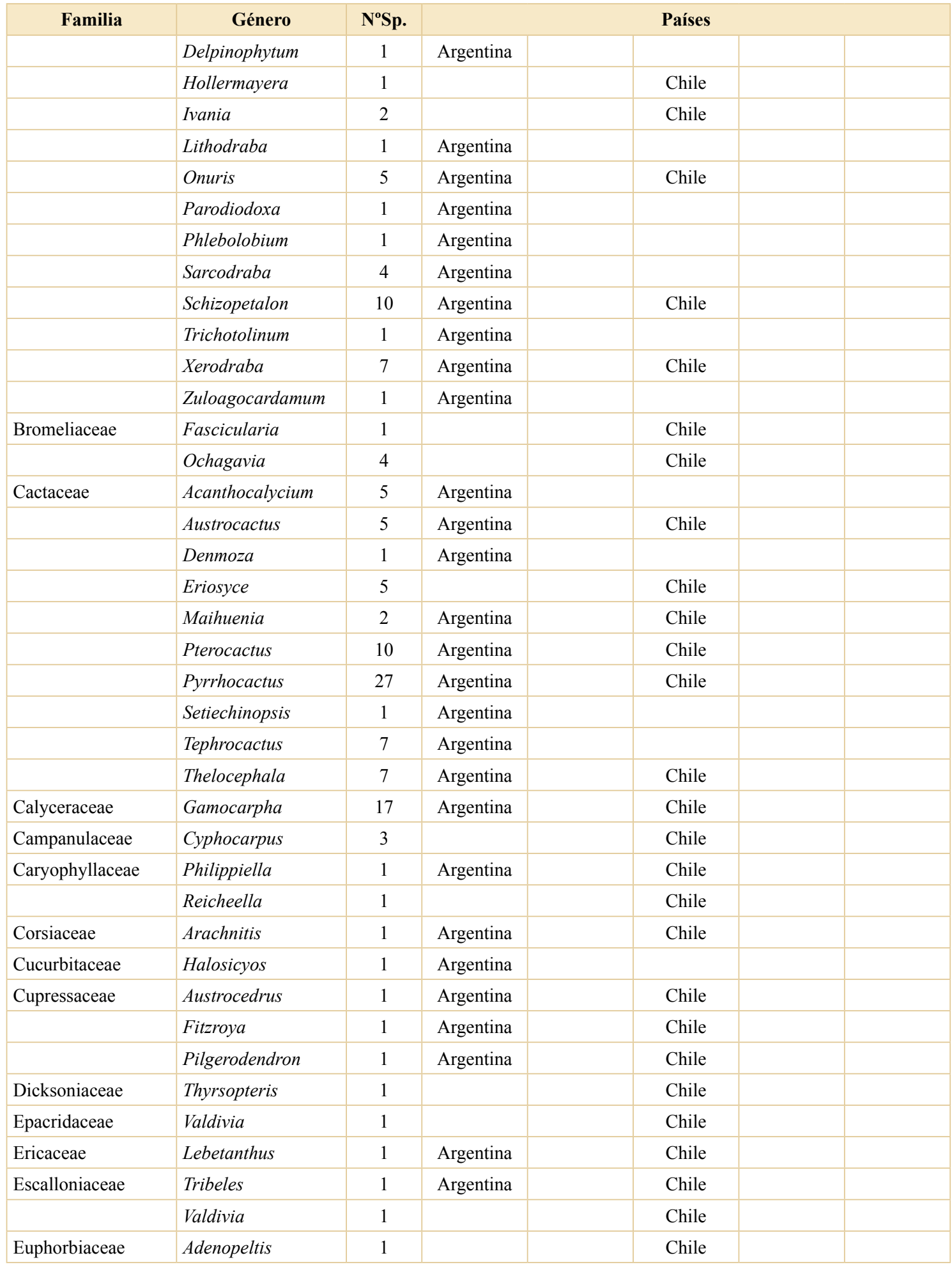


Tabla 5. (Continuación).

\begin{tabular}{|c|c|c|c|c|c|c|c|}
\hline \multirow[t]{2}{*}{ Familia } & \multirow{2}{*}{\begin{tabular}{|l|} 
Género \\
Avellanita
\end{tabular}} & \multirow{2}{*}{$\begin{array}{c}\text { NoSp. } \\
1\end{array}$} & \multicolumn{5}{|c|}{ Países } \\
\hline & & & & & Chile & & \\
\hline & Colliguaja & 5 & Argentina & S Brasil & Chile & & \\
\hline & Dysopsis & 2 & Argentina & & Chile & & \\
\hline & Spegazziniophytum & 1 & Argentina & & & & \\
\hline \multirow[t]{7}{*}{ Fabaceae } & Anarthrophyllum & 15 & Argentina & & Chile & & \\
\hline & Balsamocarpon & 1 & Argentina & & Chile & & \\
\hline & Lophocarpinia & 1 & Argentina & & & Paraguay & \\
\hline & Ramorinoa & 1 & Argentina & & & & \\
\hline & Sellocharis & 1 & & S Brasil & & & \\
\hline & Stenodrepanum & 1 & Argentina & & & & \\
\hline & Zuccagnia & 1 & Argentina & & & & \\
\hline \multirow[t]{5}{*}{ Francoaceae } & Araeoandra & 1 & & & Chile & & \\
\hline & Cissarobryon & 1 & & & Chile & & \\
\hline & Francoa & 1 & & & Chile & & \\
\hline & Tetilla & 1 & & & Chile & & \\
\hline & Viviania & 4 & Argentina & S Brasil & Chile & & \\
\hline \multirow[t]{3}{*}{ Gesneriaceae } & Asteranthera & 1 & Argentina & & Chile & & \\
\hline & Mitraria & 1 & Argentina & & Chile & & \\
\hline & Sarmienta & 1 & & & Chile & & \\
\hline Gomortegaceae & Gomortega & 1 & & & Chile & & \\
\hline Halophytaceae & Halophytum & 1 & Argentina & & & & \\
\hline \multirow[t]{3}{*}{ Iridaceae } & Onira & 1 & & S Brasil & & & Uruguay \\
\hline & Solenomelus & 2 & Argentina & & Chile & & \\
\hline & Tapeinia & 1 & Argentina & & & & \\
\hline Juncaginaceae & Tetroncium & 1 & Argentina & & Chile & & \\
\hline \multirow[t]{2}{*}{ Lamiaceae } & Cuminia & 1 & & & Chile & & \\
\hline & Kurzamra & 1 & Argentina & & Chile & & \\
\hline \multirow[t]{2}{*}{ Lardizabalaceae } & Boquila & 1 & Argentina & & Chile & & \\
\hline & Lardizabala & 1 & Argentina & & Chile & & \\
\hline \multirow[t]{4}{*}{ Loasaceae } & Grausa & 5 & Argentina & & Chile & & \\
\hline & Huidobria & 2 & & & Chile & & \\
\hline & Pinnasa & 4 & Argentina & & Chile & & \\
\hline & Scyphanthus & 1 & & & Chile & & \\
\hline \multirow[t]{2}{*}{ Loranthaceae } & Desmaria & 1 & & & Chile & & \\
\hline & Notanthera & 1 & & & Chile & & \\
\hline \multirow[t]{4}{*}{ Malpighiaceae } & Cordobia & 1 & Argentina & & & Paraguay & \\
\hline & Dinemagonum & 1 & & & Chile & & \\
\hline & Dinemandra & 1 & & & Chile & & \\
\hline & Gallardoa & 1 & Argentina & & & & \\
\hline
\end{tabular}


Tabla 5. (Continuación).

\begin{tabular}{|c|c|c|c|c|c|c|c|}
\hline \multirow[t]{2}{*}{ Familia } & \multirow{2}{*}{$\begin{array}{l}\text { Género } \\
\text { Tricomaria }\end{array}$} & \multirow{2}{*}{$\begin{array}{c}\mathbf{N}^{\mathbf{o}} \mathbf{S p} . \\
1\end{array}$} & \multicolumn{5}{|c|}{ Países } \\
\hline & & & Argentina & & & & \\
\hline \multirow[t]{7}{*}{ Malvaceae } & Bordasia & 1 & & & & Paraguay & \\
\hline & Calyculogygas & 1 & & S Brasil & & & Uruguay \\
\hline & Calyptraemalva & 1 & & S Brasil & & & \\
\hline & Corynabutilon & 7 & Argentina & & Chile & & \\
\hline & Lecanophora & 7 & Argentina & & Chile & & \\
\hline & Neobaclea & 1 & Argentina & & & & \\
\hline & Tropidococcus & 1 & & S Brasil & & & \\
\hline Marsileaceae & Regnellidium & 1 & Argentina & S Brasil & & & Uruguay \\
\hline Misodendraceae & Misodendrum & 8 & Argentina & & Chile & & \\
\hline Monimiaceae & Peumus & 1 & & & Chile & & \\
\hline \multirow[t]{2}{*}{ Montiaceae } & Lenzia & 1 & Argentina & & Chile & & \\
\hline & Schreiteria & 1 & Argentina & & & & \\
\hline \multirow[t]{3}{*}{ Myrtaceae } & Amomyrtus & 2 & Argentina & & Chile & & \\
\hline & Legrandia & 1 & & & Chile & & \\
\hline & Luma & 2 & Argentina & & Chile & & \\
\hline Nanodeaceae & Nanodea & 1 & Argentina & & Chile & & \\
\hline \multirow[t]{3}{*}{ Orchidaceae } & Bipinnula & 10 & Argentina & S Brasil & Chile & & Uruguay \\
\hline & Codonorchis & 2 & Argentina & S Brasil & Chile & & \\
\hline & Gavilea & 17 & Argentina & & Chile & & \\
\hline \multirow[t]{2}{*}{ Philesiaceae } & Lapageria & 1 & & & Chile & & \\
\hline & Philesia & 1 & Argentina & & Chile & & \\
\hline \multirow[t]{2}{*}{ Phytolacacceae } & Anisomeria & 2 & & & Chile & & \\
\hline & Ercilla & 2 & & & Chile & & \\
\hline \multirow[t]{3}{*}{ Plantaginaceae } & Fonkia & 1 & Argentina & & Chile & & \\
\hline & Melosperma & 2 & Argentina & & Chile & & \\
\hline & Monttea & 3 & Argentina & & Chile & & \\
\hline \multirow[t]{10}{*}{ Poaceae } & Cambajuva & 1 & & S Brasil & & & \\
\hline & Eremium & 1 & Argentina & & Chile & & \\
\hline & Leptophyllochloa & 1 & Argentina & & Chile & & \\
\hline & Megalachne & 3 & & & Chile & & \\
\hline & Neobouteloua & 2 & Argentina & & & & \\
\hline & Nicoraepoa & 6 & Argentina & & Chile & & \\
\hline & Oplismenopsis & 1 & Argentina & & & & Uruguay \\
\hline & Osvaldoa & 1 & Argentina & & & & \\
\hline & Podophorus & 1 & & & Chile & & \\
\hline & Relchela & 1 & Argentina & & Chile & & \\
\hline \multirow[t]{2}{*}{ Podocarpaceae } & Lepidothamnus & 1 & Argentina & & Chile & & \\
\hline & Saxegothaea & 1 & Argentina & & Chile & & \\
\hline
\end{tabular}


Tabla 5. (Continuación).

\begin{tabular}{|c|c|c|c|c|c|c|c|}
\hline Familia & Género & $\mathrm{N}^{\circ} \mathrm{Sp}$. & & & Países & & \\
\hline Podostemaceae & Heterotristicha & 1 & & & & & Uruguay \\
\hline Polypodiaceae & Synammia & 3 & & & Chile & & \\
\hline Proteaceae & Gevuina & 1 & Argentina & & Chile & & \\
\hline Ranunculaceae & Barneoudia & 3 & Argentina & & Chile & & \\
\hline & Callianthemoides & 1 & Argentina & & Chile & & \\
\hline & Hamadryas & 5 & Argentina & & Chile & & \\
\hline Rhamnaceae & Ochetophila & 2 & Argentina & & Chile & & \\
\hline & Retanilla & 4 & Argentina & & Chile & & \\
\hline & Trevoa & 1 & & & Chile & & \\
\hline Rosaceae & Margyracaena & 1 & & & Chile & & \\
\hline Rubiaceae & Cruckshanksia & 7 & Argentina & & Chile & & \\
\hline & Oreopolus & 1 & Argentina & & Chile & & \\
\hline Rutaceae & Pitavia & 1 & & & Chile & & \\
\hline & Raulinoa & 1 & & S Brasil & & & \\
\hline Santalaceae & Myoschilos & 1 & Argentina & & Chile & & \\
\hline Sapindaceae & Bridgesia & 1 & & & Chile & & \\
\hline & Guindilia & 3 & Argentina & & Chile & & \\
\hline Saxifragaceae & Saxifragella & 1 & Argentina & & Chile & & \\
\hline & Saxifragodes & 1 & Argentina & & Chile & & \\
\hline Solanaceae & Benthamiella & 12 & Argentina & & Chile & & \\
\hline & Combera & 2 & Argentina & & Chile & & \\
\hline & Eriolarynx & 2 & Argentina & & & & \\
\hline & Latua & 1 & & & Chile & & \\
\hline & Pantacantha & 1 & Argentina & & & & \\
\hline & Reyesia & 4 & Argentina & & Chile & & \\
\hline & Salpiglossis & 2 & Argentina & & Chile & & \\
\hline & Schizanthus & 13 & Argentina & & Chile & & \\
\hline & Sclerophylax & 14 & Argentina & & & Paraguay & Uruguay \\
\hline & Vestia & 1 & & & Chile & & \\
\hline Tecophilaeaceae & Conanthera & 5 & & & Chile & & \\
\hline & Tecophilaea & 2 & & & Chile & & \\
\hline Verbenaceae & Diostea & 1 & Argentina & & Chile & & \\
\hline & Dipyrena & 1 & Argentina & & & & \\
\hline & Neosparton & 3 & Argentina & & & & \\
\hline & Parodianthus & 2 & Argentina & & & & \\
\hline Zygophyllaceae & Metharme & 1 & & & Chile & & \\
\hline & Pintoa & 1 & & & Chile & & \\
\hline & Plectrocarpa & 2 & Argentina & & & & \\
\hline
\end{tabular}




\section{Flora adventicia}

Las familias introducidas en el Cono Sur son 9: Aponogetonaceae, Balsaminaceae, Elaeagnaceae, Mazaceae, Musaceae, Pinaceae, Pittosporaceae, Resedaceae y Sphenocleaceae.

En cuanto a los géneros introducidos, en el Cono Sur habitan 397, pertenecientes a 85 familias (Tabla 6). El mayor número de géneros introducidos se encuentra en las Asteraceae (59), Poaceae (47) y Fabaceae (28); en números relativos es importante la proporción de géneros introducidos en familias como las Apiaceae (19 géneros introducidos de 43), Brassicaceae (30 de 71) y Lamiaceae (16 de 49).

El número de especies introducidas en el Cono Sur es de 1326, lo que representa el 7\% de su flora.

Tabla 6. Géneros introducidos, número de especies en el Cono Sur y distribución por países.

\begin{tabular}{|c|c|c|c|c|c|c|c|}
\hline \multirow{2}{*}{\begin{tabular}{|r|} 
Familia \\
Acanthaceae \\
\end{tabular}} & \multirow{2}{*}{\begin{tabular}{|l|} 
Género \\
Barleria
\end{tabular}} & \multirow{2}{*}{$\begin{array}{c}\mathbf{N}^{\mathbf{o}} \mathbf{S p} . \\
1\end{array}$} & \multicolumn{5}{|c|}{ Países } \\
\hline & & & & S Brasil & & & \\
\hline & Hypoestes & 1 & & & & Paraguay & \\
\hline & Odontonema & 1 & & & & Paraguay & \\
\hline & Thunbergia & 3 & Argentina & S Brasil & & Paraguay & \\
\hline Aceraceae & Acer & 2 & Argentina & & & & \\
\hline Achariaceae & Hydnocarpus & 1 & & & & Paraguay & \\
\hline \multirow[t]{6}{*}{ Aizoaceae } & Aptenia & 1 & Argentina & & & & \\
\hline & Cypselea & 1 & Argentina & & & & \\
\hline & Galenia & 1 & & & Chile & & \\
\hline & Malephora & 1 & Argentina & S Brasil & & & \\
\hline & Mesembryanthemum & 2 & Argentina & & Chile & & \\
\hline & Sesuvium & 1 & Argentina & S Brasil & Chile & Paraguay & Uruguay \\
\hline Alismataceae & Alisma & 2 & Argentina & & Chile & & \\
\hline \multirow[t]{6}{*}{ Amaryllidaceae } & Agapanthus & 1 & Argentina & & & & \\
\hline & Allium & 7 & Argentina & & Chile & & \\
\hline & Amaryllis & 1 & & S Brasil & Chile & Paraguay & \\
\hline & Brunsvigia & 1 & Argentina & & & & \\
\hline & Leucojum & 1 & & & Chile & & \\
\hline & Pyrolirion & 1 & & & Chile & & \\
\hline Anacardiaceae & Mangifera & 1 & & S Brasil & & Paraguay & \\
\hline \multirow[t]{10}{*}{ Apiaceae } & $A m m i$ & 2 & Argentina & S Brasil & Chile & & Uruguay \\
\hline & Anethum & 1 & Argentina & S Brasil & Chile & & \\
\hline & Anthriscus & 2 & Argentina & & Chile & & \\
\hline & Bupleurum & 1 & Argentina & & & & \\
\hline & Caucalis & 1 & Argentina & & & & \\
\hline & Conium & 1 & Argentina & S Brasil & Chile & & Uruguay \\
\hline & Coriandrum & 1 & Argentina & S Brasil & Chile & Paraguay & \\
\hline & Foeniculum & 1 & Argentina & S Brasil & Chile & Paraguay & Uruguay \\
\hline & Heracleum & 1 & Argentina & & & & \\
\hline & Levisticum & 1 & & & Chile & & \\
\hline
\end{tabular}


Tabla 6. (Continuación).

\begin{tabular}{|c|c|c|c|c|c|c|c|}
\hline \multirow[t]{2}{*}{ Familia } & \multirow{2}{*}{$\begin{array}{l}\text { Género } \\
\text { Oenanthe }\end{array}$} & \multirow{2}{*}{$\begin{array}{c}\mathbf{N}^{\mathbf{o}} \mathbf{S p} \text {. } \\
2\end{array}$} & \multicolumn{5}{|c|}{ Países } \\
\hline & & & Argentina & & & & Uruguay \\
\hline & Pastinaca & 1 & Argentina & & Chile & & Uruguay \\
\hline & Petroselinum & 1 & Argentina & S Brasil & Chile & & \\
\hline & Pimpinella & 1 & Argentina & S Brasil & & & \\
\hline & Scandix & 1 & Argentina & & Chile & & \\
\hline & Seseli & 1 & & & Chile & & \\
\hline & Sium & 1 & & & Chile & & \\
\hline & Tordylium & 1 & Argentina & & & & \\
\hline & Torilis & 2 & Argentina & S Brasil & Chile & & Uruguay \\
\hline \multirow[t]{5}{*}{ Apocynaceae } & Calotropis & 1 & & & & Paraguay & \\
\hline & Catharanthus & 1 & Argentina & S Brasil & Chile & Paraguay & Uruguay \\
\hline & Gomphocarpus & 1 & & S Brasil & & & \\
\hline & Plumeria & 1 & Argentina & S Brasil & & & \\
\hline & Vinca & 1 & Argentina & S Brasil & Chile & & Uruguay \\
\hline Aponogetonaceae & Aponogeton & 1 & & & Chile & & \\
\hline \multirow[t]{7}{*}{ Araceae } & Alocasia & 1 & & & & Paraguay & \\
\hline & Arum & 1 & Argentina & & & & Uruguay \\
\hline & Colocasia & 1 & Argentina & S Brasil & Chile & Paraguay & Uruguay \\
\hline & Landoltia & 1 & & S Brasil & Chile & & \\
\hline & Syngonium & 2 & & S Brasil & & & \\
\hline & Typhonium & 1 & & S Brasil & & & \\
\hline & Zantedeschia & 1 & Argentina & S Brasil & Chile & & Uruguay \\
\hline \multirow[t]{2}{*}{ Araliaceae } & Hedera & 1 & Argentina & & Chile & & \\
\hline & Tetrapanax & 1 & & S Brasil & & & \\
\hline \multirow[t]{2}{*}{ Arecaceae } & Phoenix & 1 & Argentina & & & & \\
\hline & Trachycarpus & 1 & Argentina & & & & \\
\hline \multirow[t]{6}{*}{ Asparagaceae } & Agave & 1 & Argentina & & & & \\
\hline & Asparagus & 6 & Argentina & S Brasil & & & Uruguay \\
\hline & Beschorneria & 1 & Argentina & & & & \\
\hline & Cordyline & 2 & Argentina & S Brasil & Chile & Paraguay & Uruguay \\
\hline & Ophiopogon & 1 & Argentina & & & & \\
\hline & Yucca & 2 & Argentina & & & & \\
\hline \multirow[t]{2}{*}{ Asphodelaceae } & Asphodelus & 1 & Argentina & & Chile & & \\
\hline & Kniphofia & 1 & Argentina & & & & \\
\hline \multirow[t]{2}{*}{ Aspleniaceae } & Ceterach & 1 & Argentina & & & & \\
\hline & Phyllitis & 1 & Argentina & & & & \\
\hline \multirow[t]{2}{*}{ Asteraceae } & Achillea & 2 & Argentina & S Brasil & Chile & & Uruguay \\
\hline & Anthemis & 2 & Argentina & S Brasil & Chile & Paraguay & Uruguay \\
\hline
\end{tabular}


Tabla 6. (Continuación).

\begin{tabular}{|c|c|c|c|c|c|c|c|}
\hline \multirow[t]{2}{*}{ Familia } & \multirow{2}{*}{$\begin{array}{l}\text { Género } \\
\text { Arctium }\end{array}$} & \multirow{2}{*}{$\begin{array}{c}\mathbf{N}^{\mathbf{o}} \mathbf{S p} . \\
1\end{array}$} & \multicolumn{5}{|c|}{ Países } \\
\hline & & & Argentina & S Brasil & Chile & & Uruguay \\
\hline & Arctotheca & 1 & Argentina & & Chile & & \\
\hline & Arctotis & 1 & Argentina & S Brasil & & & Uruguay \\
\hline & Argyranthemum & 1 & Argentina & & Chile & & \\
\hline & Arnica & 1 & Argentina & & Chile & & \\
\hline & Bellis & 1 & Argentina & & Chile & & \\
\hline & Brachylaena & 1 & & S Brasil & & & \\
\hline & Calendula & 3 & Argentina & & Chile & & Uruguay \\
\hline & Carduus & 5 & Argentina & S Brasil & Chile & & Uruguay \\
\hline & Carthamus & 2 & Argentina & S Brasil & Chile & & Uruguay \\
\hline & Chondrilla & 1 & Argentina & & & & \\
\hline & Chrysanthemoides & 1 & & & Chile & & \\
\hline & Chrysanthemum & 2 & Argentina & & Chile & & Uruguay \\
\hline & Cichorium & 1 & Argentina & S Brasil & Chile & & Uruguay \\
\hline & Cirsium & 2 & Argentina & S Brasil & Chile & Paraguay & Uruguay \\
\hline & Cladanthus & 1 & Argentina & S Brasil & Chile & & Uruguay \\
\hline & Coleostephus & 1 & Argentina & S Brasil & Chile & & Uruguay \\
\hline & Crepis & 5 & Argentina & S Brasil & Chile & & Uruguay \\
\hline & Cynara & 1 & Argentina & S Brasil & Chile & & Uruguay \\
\hline & Dahlia & 1 & Argentina & & & & \\
\hline & Delairea & 1 & Argentina & & Chile & & Uruguay \\
\hline & Dittrichia & 1 & & & Chile & & \\
\hline & Dyssodia & 1 & Argentina & & & & \\
\hline & Eleutheranthera & 1 & & S Brasil & & & \\
\hline & Emilia & 2 & Argentina & S Brasil & & Paraguay & \\
\hline & Euryops & 1 & Argentina & & & & \\
\hline & Gazania & 1 & Argentina & & & & \\
\hline & Gynura & 1 & Argentina & & & & \\
\hline & Hedypnois & 1 & Argentina & & Chile & & Uruguay \\
\hline & Helianthus & 4 & Argentina & & Chile & & Uruguay \\
\hline & Helichrysum & 1 & & S Brasil & & & \\
\hline & Helminthotheca & 1 & Argentina & & Chile & & Uruguay \\
\hline & Heterotheca & 1 & Argentina & & & & \\
\hline & Lactuca & 5 & Argentina & S Brasil & Chile & Paraguay & Uruguay \\
\hline & Lapsana & 1 & Argentina & S Brasil & Chile & & \\
\hline & Leontodon & 3 & Argentina & & Chile & & \\
\hline & Leucanthemum & 1 & Argentina & S Brasil & Chile & & Uruguay \\
\hline & Logfia & 1 & & & Chile & & \\
\hline
\end{tabular}


Tabla 6. (Continuación).

\begin{tabular}{|c|c|c|c|c|c|c|c|}
\hline \multirow[t]{2}{*}{ Familia } & \multirow{2}{*}{\begin{tabular}{|l} 
Género \\
Matricaria
\end{tabular}} & \multirow{2}{*}{$\begin{array}{c}\mathbf{N}^{\mathbf{o}} \mathbf{S p} . \\
2\end{array}$} & \multicolumn{5}{|c|}{ Países } \\
\hline & & & Argentina & S Brasil & Chile & Paraguay & Uruguay \\
\hline & Microseris & 1 & & & Chile & & \\
\hline & Montanoa & 1 & Argentina & & & & \\
\hline & Onopordum & 2 & Argentina & S Brasil & Chile & & Uruguay \\
\hline & Ratibida & 1 & Argentina & & & & \\
\hline & Rhaponticum & 1 & Argentina & & & & \\
\hline & Scolymus & 1 & Argentina & & Chile & & \\
\hline & Scorzonera & 1 & Argentina & & & & \\
\hline & Silybum & 1 & Argentina & S Brasil & Chile & & Uruguay \\
\hline & Struchium & 1 & & S Brasil & & & \\
\hline & Tanacetum & 3 & Argentina & S Brasil & Chile & & Uruguay \\
\hline & Tithonia & 3 & Argentina & S Brasil & & & \\
\hline & Tolpis & 1 & & & Chile & & \\
\hline & Tragopogon & 3 & Argentina & & Chile & & \\
\hline & Tripleurospermum & 1 & Argentina & & Chile & & Uruguay \\
\hline & Urospermum & 1 & Argentina & & Chile & & Uruguay \\
\hline & Volutaria & 1 & & & Chile & & \\
\hline & Xerochrysum & 1 & & S Brasil & & & \\
\hline & Youngia & 1 & Argentina & & & & \\
\hline Athyriaceae & Deparia & 1 & Argentina & S Brasil & & Paraguay & \\
\hline Balsaminaceae & Impatiens & 3 & Argentina & S Brasil & & & \\
\hline Betulaceae & Betula & 1 & Argentina & & & & \\
\hline \multirow[t]{2}{*}{ Bignoniaceae } & Campsis & 1 & Argentina & & & & \\
\hline & Podranea & 1 & Argentina & S Brasil & & & \\
\hline \multirow[t]{10}{*}{ Boraginaceae } & Anchusa & 1 & Argentina & & & & \\
\hline & Asperugo & 1 & Argentina & & Chile & & \\
\hline & Borago & 1 & Argentina & & Chile & & Uruguay \\
\hline & Buglossoides & 1 & Argentina & & Chile & & Uruguay \\
\hline & Cynoglossum & 3 & Argentina & S Brasil & Chile & & \\
\hline & Echium & 2 & Argentina & S Brasil & Chile & & Uruguay \\
\hline & Lithospermum & 1 & Argentina & & & & \\
\hline & Lycopsis & 1 & Argentina & & & & \\
\hline & Omphalodes & 1 & & & Chile & & \\
\hline & Symphytum & 1 & Argentina & & & & \\
\hline \multirow[t]{4}{*}{ Brassicaceae } & Alliaria & 1 & Argentina & & & & \\
\hline & Alyssum & 1 & Argentina & & Chile & & \\
\hline & Arabidopsis & 1 & Argentina & & Chile & & Uruguay \\
\hline & Barbarea & 2 & Argentina & & Chile & & \\
\hline
\end{tabular}


Tabla 6. (Continuación).

\begin{tabular}{|c|c|c|c|c|c|c|c|}
\hline \multirow[t]{2}{*}{ Familia } & \multirow{2}{*}{$\begin{array}{l}\text { Género } \\
\text { Brassica }\end{array}$} & \multirow{2}{*}{$\begin{array}{c}\mathbf{N}^{\circ} \mathbf{S p} . \\
6\end{array}$} & \multicolumn{5}{|c|}{ Países } \\
\hline & & & Argentina & S Brasil & Chile & Paraguay & Uruguay \\
\hline & Cakile & 1 & Argentina & & & & Uruguay \\
\hline & Camelina & 3 & Argentina & & Chile & & Uruguay \\
\hline & Capsella & 1 & Argentina & S Brasil & Chile & & Uruguay \\
\hline & Chorispora & 1 & Argentina & & Chile & & \\
\hline & Diplotaxis & 3 & Argentina & & Chile & & Uruguay \\
\hline & Eruca & 1 & Argentina & & Chile & & \\
\hline & Erysimum & 2 & Argentina & & & & \\
\hline & Hesperis & 1 & Argentina & & Chile & & \\
\hline & Hirschfeldia & 1 & Argentina & & Chile & & Uruguay \\
\hline & Hornungia & 1 & Argentina & & Chile & & \\
\hline & Iberis & 1 & Argentina & & & & \\
\hline & Isatis & 1 & & & Chile & & \\
\hline & Lobularia & 1 & Argentina & & Chile & & Uruguay \\
\hline & Lunaria & 1 & Argentina & & & & \\
\hline & Malcolmia & 1 & Argentina & & & & \\
\hline & Matthiola & 1 & & & Chile & & \\
\hline & Nasturtium & 2 & Argentina & S Brasil & Chile & Paraguay & Uruguay \\
\hline & Neslia & 1 & Argentina & & & & \\
\hline & Raphanus & 2 & Argentina & S Brasil & Chile & Paraguay & Uruguay \\
\hline & Rapistrum & 1 & Argentina & S Brasil & Chile & Paraguay & Uruguay \\
\hline & Sinapis & 2 & Argentina & S Brasil & Chile & Paraguay & \\
\hline & Sisymbrium & 7 & Argentina & & Chile & & Uruguay \\
\hline & Strigosella & 1 & Argentina & & & & \\
\hline & Teesdalia & 1 & & & Chile & & \\
\hline & Thlaspi & 1 & Argentina & & Chile & & \\
\hline \multirow[t]{2}{*}{ Cactaceae } & Cylindropuntia & 2 & Argentina & & Chile & & \\
\hline & Nopalea & 1 & & S Brasil & & Paraguay & \\
\hline \multirow[t]{2}{*}{ Campanulaceae } & Campanula & 2 & Argentina & & Chile & & \\
\hline & Hippobroma & 1 & & S Brasil & & & \\
\hline Cannabaceae & Humulus & 2 & Argentina & & & & \\
\hline \multirow[t]{7}{*}{ Caprifoliaceae } & Centranthus & 1 & Argentina & & Chile & & \\
\hline & Dipsacus & 2 & Argentina & & Chile & & Uruguay \\
\hline & Knautia & 2 & Argentina & & Chile & & \\
\hline & Lonicera & 1 & Argentina & S Brasil & & & Uruguay \\
\hline & Scabiosa & 2 & Argentina & S Brasil & Chile & & Uruguay \\
\hline & Symphoricarpos & 1 & Argentina & & & & \\
\hline & Valerianella & 4 & Argentina & & Chile & & \\
\hline
\end{tabular}


Tabla 6. (Continuación).

\begin{tabular}{|c|c|c|c|c|c|c|c|}
\hline \multirow{2}{*}{$\begin{array}{c}\text { Familia } \\
\text { Caryophyllaceae }\end{array}$} & \multirow{2}{*}{$\begin{array}{r}\text { Género } \\
\text { Agrostemma }\end{array}$} & \multirow{2}{*}{$\begin{array}{c}\text { NoSp. } \\
1\end{array}$} & \multicolumn{5}{|c|}{ Países } \\
\hline & & & Argentina & S Brasil & Chile & & Uruguay \\
\hline & Dianthus & 2 & Argentina & & Chile & & \\
\hline & Holosteum & 1 & Argentina & & & & \\
\hline & Lychnis & 1 & & S Brasil & Chile & & \\
\hline & Petrorhagia & 3 & Argentina & & Chile & & \\
\hline & Saponaria & 1 & Argentina & & Chile & & Uruguay \\
\hline & Scleranthus & 1 & Argentina & & Chile & & Uruguay \\
\hline & Vaccaria & 1 & Argentina & S Brasil & & & Uruguay \\
\hline \multirow[t]{7}{*}{ Chenopodiaceae } & Bassia & 2 & Argentina & & Chile & & \\
\hline & Beta & 1 & Argentina & & Chile & & Uruguay \\
\hline & Blitum & 1 & Argentina & & & & \\
\hline & Chenopodiastrum & 1 & Argentina & & Chile & & Uruguay \\
\hline & Cycloloma & 1 & Argentina & & & & \\
\hline & Maireana & 1 & & & Chile & & \\
\hline & Salsola & 2 & Argentina & & Chile & & Uruguay \\
\hline \multirow[t]{2}{*}{ Crassulaceae } & Bryophyllum & 4 & Argentina & S Brasil & & & \\
\hline & Kalanchoe & 1 & & S Brasil & & & \\
\hline \multirow[t]{4}{*}{ Cucurbitaceae } & Citrullus & 2 & Argentina & S Brasil & & Paraguay & \\
\hline & Cucumis & 3 & Argentina & S Brasil & & Paraguay & \\
\hline & Lagenaria & 1 & Argentina & S Brasil & & Paraguay & Uruguay \\
\hline & Luffa & 1 & Argentina & S Brasil & & Paraguay & \\
\hline \multirow[t]{2}{*}{ Cupressaceae } & Cupressus & 2 & Argentina & & & & \\
\hline & Juniperus & 1 & Argentina & & & & \\
\hline Dryopteridaceae & Cyrtomium & 1 & Argentina & & & & \\
\hline Elaeagnaceae & Elaeagnus & 1 & Argentina & & & & \\
\hline \multirow[t]{5}{*}{ Euphorbiaceae } & Aleurites & 1 & Argentina & & & & \\
\hline & Breynia & 1 & & S Brasil & & & \\
\hline & Mercurialis & 1 & Argentina & & Chile & & \\
\hline & Ricinus & 1 & Argentina & S Brasil & Chile & Paraguay & Uruguay \\
\hline & Vernicia & 1 & Argentina & & & & \\
\hline \multirow[t]{8}{*}{ Fabaceae } & Adenocarpus & 1 & & & & & Uruguay \\
\hline & Alysicarpus & 2 & & S Brasil & & & \\
\hline & Amorpha & 1 & Argentina & & & & Uruguay \\
\hline & Caesalpinia & 2 & & S Brasil & & Paraguay & \\
\hline & Cajanus & 1 & & S Brasil & & Paraguay & \\
\hline & Colutea & 1 & Argentina & & & & \\
\hline & Coronilla & 1 & Argentina & & & & \\
\hline & Cytisus & 2 & Argentina & & Chile & & \\
\hline
\end{tabular}


Tabla 6. (Continuación).

\begin{tabular}{|c|c|c|c|c|c|c|c|}
\hline \multirow[t]{2}{*}{ Familia } & \multirow{2}{*}{$\begin{array}{l}\text { Género } \\
\text { Dipogon }\end{array}$} & \multirow{2}{*}{$\begin{array}{c}\mathbf{N}^{\mathbf{o}} \mathbf{S p} . \\
1\end{array}$} & \multicolumn{5}{|c|}{ Países } \\
\hline & & & & & Chile & & \\
\hline & Galega & 1 & Argentina & & Chile & & Uruguay \\
\hline & Genista & 2 & Argentina & & Chile & & Uruguay \\
\hline & Kummerowia & 1 & Argentina & & & & \\
\hline & Lablab & 1 & & S Brasil & Chile & & Uruguay \\
\hline & Laburnum & 1 & Argentina & & & & \\
\hline & Leucaena & 1 & Argentina & S Brasil & Chile & & \\
\hline & Medicago & 10 & Argentina & S Brasil & Chile & & Uruguay \\
\hline & Melilotus & 4 & Argentina & S Brasil & Chile & Paraguay & Uruguay \\
\hline & Neonotonia & 1 & Argentina & S Brasil & & Paraguay & \\
\hline & Ononis & 1 & & & & & Uruguay \\
\hline & Psophocarpus & 1 & & S Brasil & & & \\
\hline & Psoralea & 1 & & & & & Uruguay \\
\hline & Pueraria & 1 & Argentina & & & & \\
\hline & Racosperma & 4 & Argentina & S Brasil & Chile & & Uruguay \\
\hline & Robinia & 1 & & & Chile & & Uruguay \\
\hline & Spartium & 1 & Argentina & & Chile & & Uruguay \\
\hline & Sphaerophysa & 1 & Argentina & & & & \\
\hline & Trigonella & 1 & Argentina & & Chile & & \\
\hline & Ulex & 1 & Argentina & S Brasil & Chile & & Uruguay \\
\hline Fagaceae & Quercus & 1 & & & Chile & & \\
\hline \multirow[t]{2}{*}{ Gentianaceae } & Blackstonia & 1 & Argentina & & & & Uruguay \\
\hline & Irlbachia & 1 & & & & Paraguay & \\
\hline Hypoxidaceae & Molineria & 1 & Argentina & & & & \\
\hline \multirow[t]{9}{*}{ Iridaceae } & Belamcanda & 1 & & & & Paraguay & \\
\hline & Chasmanthe & 1 & Argentina & & & & \\
\hline & Crocosmia & 1 & Argentina & S Brasil & Chile & & \\
\hline & Freesia & 2 & Argentina & & & & \\
\hline & Gladiolus & 3 & Argentina & & & & \\
\hline & Iris & 4 & Argentina & & Chile & & Uruguay \\
\hline & Romulea & 1 & & & Chile & & \\
\hline & Sparaxis & 2 & Argentina & & & & \\
\hline & Watsonia & 3 & Argentina & & & & \\
\hline Juglandaceae & Carya & 1 & Argentina & & & & \\
\hline \multirow[t]{4}{*}{ Lamiaceae } & Ballota & 1 & Argentina & & & & Uruguay \\
\hline & Galeopsis & 1 & & & Chile & & \\
\hline & Glechoma & 1 & Argentina & & Chile & & \\
\hline & Lamium & 2 & Argentina & & Chile & & Uruguay \\
\hline
\end{tabular}


Tabla 6. (Continuación).

\begin{tabular}{|c|c|c|c|c|c|c|c|}
\hline \multirow[t]{2}{*}{ Familia } & \multirow{2}{*}{\begin{tabular}{|l|} 
Género \\
Leonotis
\end{tabular}} & \multirow{2}{*}{$\begin{array}{c}\text { No'Sp. }^{\circ} \text {. } \\
1\end{array}$} & \multicolumn{5}{|c|}{ Países } \\
\hline & & & Argentina & S Brasil & & Paraguay & Uruguay \\
\hline & Leonurus & 2 & Argentina & S Brasil & & Paraguay & Uruguay \\
\hline & Leucas & 1 & & S Brasil & & & \\
\hline & Lycopus & 1 & & & Chile & & \\
\hline & Marrubium & 1 & Argentina & S Brasil & Chile & & Uruguay \\
\hline & Melissa & 1 & Argentina & & Chile & & Uruguay \\
\hline & Mentha & 5 & Argentina & S Brasil & Chile & Paraguay & Uruguay \\
\hline & Molucella & 1 & Argentina & & Chile & & \\
\hline & Nepeta & 1 & Argentina & & & & \\
\hline & Origanum & 1 & & & Chile & & \\
\hline & Prunella & 2 & Argentina & S Brasil & Chile & & Uruguay \\
\hline & Satureja & 1 & & & Chile & & \\
\hline Liliaceae & Lilium & 1 & Argentina & S Brasil & Chile & & Uruguay \\
\hline Linderniaceae & Torenia & 1 & & S Brasil & & & \\
\hline \multirow[t]{4}{*}{ Malvaceae } & Alcea & 1 & Argentina & & & & \\
\hline & Gossypium & 3 & & S Brasil & Chile & & \\
\hline & Malva & 8 & Argentina & S Brasil & Chile & Paraguay & Uruguay \\
\hline & Pachira & 1 & & S Brasil & & & \\
\hline Mazaceae & Mazus & 1 & Argentina & S Brasil & & & \\
\hline Melastomataceae & Siphanthera & 1 & & S Brasil & & & \\
\hline Meliaceae & Melia & 1 & Argentina & S Brasil & Chile & Paraguay & Uruguay \\
\hline Montiaceae & Claytonia & 1 & Argentina & & & & \\
\hline Moraceae & Broussonetia & 1 & Argentina & & Chile & & \\
\hline Musaceae & Musa & 2 & & S Brasil & & & \\
\hline \multirow[t]{2}{*}{ Myrtaceae } & Eucalyptus & 7 & Argentina & & Chile & & \\
\hline & Syzygium & 2 & Argentina & S Brasil & Chile & & \\
\hline Nymphaeaceae & Nelumbo & 1 & & & & Paraguay & \\
\hline \multirow[t]{4}{*}{ Oleaceae } & Fraxinus & 4 & Argentina & & Chile & & \\
\hline & Jasminum & 1 & Argentina & & & & \\
\hline & Ligustrum & 4 & Argentina & S Brasil & Chile & & Uruguay \\
\hline & Syringa & 1 & Argentina & & & & \\
\hline Orchidaceae & Zeuxine & 1 & & S Brasil & & & \\
\hline \multirow[t]{2}{*}{ Orobanchaceae } & Bellardia & 2 & Argentina & S Brasil & Chile & & Uruguay \\
\hline & Parentucellia & 1 & & & Chile & & \\
\hline Papaveraceae & Chelidonium & 1 & Argentina & S Brasil & & & \\
\hline \multirow[t]{3}{*}{ Papaveraceae } & Eschscholzia & 1 & Argentina & & Chile & & \\
\hline & Fumaria & 8 & Argentina & & Chile & & Uruguay \\
\hline & Glaucium & 2 & Argentina & & & & \\
\hline
\end{tabular}


Tabla 6. (Continuación).

\begin{tabular}{|c|c|c|c|c|c|c|c|}
\hline \multirow[t]{2}{*}{ Familia } & \multirow{2}{*}{$\begin{array}{l}\text { Género } \\
\text { Papaver }\end{array}$} & \multirow{2}{*}{$\begin{array}{c}\mathbf{N}^{\circ} \mathrm{Sp} . \\
5\end{array}$} & \multicolumn{5}{|c|}{ Países } \\
\hline & & & Argentina & & Chile & & Uruguay \\
\hline \multirow[t]{2}{*}{ Pinaceae } & Pinus & 13 & Argentina & S Brasil & Chile & & \\
\hline & Pseudotsuga & 1 & Argentina & & & & \\
\hline Pittosporaceae & Pittosporum & 2 & & & Chile & & \\
\hline \multirow[t]{8}{*}{ Plantaginaceae } & Antirrhinum & 1 & Argentina & & & & Uruguay \\
\hline & Cymbalaria & 1 & Argentina & & Chile & & Uruguay \\
\hline & Digitalis & 1 & Argentina & S Brasil & Chile & & Uruguay \\
\hline & Kickxia & 1 & Argentina & & Chile & & Uruguay \\
\hline & Linaria & 3 & Argentina & & Chile & & \\
\hline & Maurandya & 3 & Argentina & S Brasil & & & \\
\hline & Misopates & 1 & Argentina & & & & Uruguay \\
\hline & Nuttallanthus & 1 & Argentina & S Brasil & Chile & & Uruguay \\
\hline Plumbaginaceae & Ceratostigma & 1 & Argentina & & & & \\
\hline \multirow[t]{25}{*}{ Poaceae } & Agropyron & 1 & & & Chile & & \\
\hline & Aira & 4 & Argentina & S Brasil & Chile & & Uruguay \\
\hline & Ammophila & 1 & Argentina & & Chile & & \\
\hline & Apera & 1 & Argentina & & Chile & & \\
\hline & Arrhenatherum & 1 & Argentina & S Brasil & Chile & & Uruguay \\
\hline & Arundo & 1 & Argentina & S Brasil & Chile & & \\
\hline & Austrostipa & 2 & Argentina & & Chile & & \\
\hline & Avena & 6 & Argentina & S Brasil & Chile & & Uruguay \\
\hline & Brachypodium & 2 & Argentina & & Chile & & Uruguay \\
\hline & Briza & 3 & Argentina & S Brasil & Chile & & Uruguay \\
\hline & Catapodium & 1 & Argentina & S Brasil & Chile & & Uruguay \\
\hline & Chrysopogon & 1 & Argentina & & & Paraguay & \\
\hline & Coix & 1 & Argentina & S Brasil & Chile & Paraguay & \\
\hline & Corynephorus & 2 & Argentina & & Chile & & \\
\hline & Cymbopogon & 3 & Argentina & S Brasil & Chile & & \\
\hline & Cynosurus & 2 & Argentina & & Chile & & Uruguay \\
\hline & Dactylis & 1 & Argentina & S Brasil & Chile & & Uruguay \\
\hline & Dactyloctenium & 1 & Argentina & S Brasil & & Paraguay & Uruguay \\
\hline & Dichanthium & 2 & Argentina & & & Paraguay & \\
\hline & Ehrharta & 3 & Argentina & & Chile & & \\
\hline & Elytrigia & 1 & Argentina & & Chile & & \\
\hline & Gastridium & 2 & Argentina & & Chile & & \\
\hline & Gaudinia & 1 & Argentina & & & & Uruguay \\
\hline & Hackelochloa & 1 & Argentina & & & Paraguay & \\
\hline & Hainardia & 1 & Argentina & S Brasil & Chile & & Uruguay \\
\hline
\end{tabular}


Tabla 6. (Continuación).

\begin{tabular}{|c|c|c|c|c|c|c|c|}
\hline \multirow[t]{2}{*}{ Familia } & \multirow{2}{*}{$\begin{array}{l}\text { Género } \\
\text { Hemarthria }\end{array}$} & \multirow{2}{*}{$\begin{array}{c}\text { NoSp. } \\
1\end{array}$} & \multicolumn{5}{|c|}{ Países } \\
\hline & & & Argentina & S Brasil & & Paraguay & Uruguay \\
\hline & Holcus & 1 & Argentina & S Brasil & Chile & & Uruguay \\
\hline & Lachnagrostis & 1 & Argentina & & Chile & & \\
\hline & Lagurus & 1 & Argentina & S Brasil & Chile & & Uruguay \\
\hline & Lamarckia & 1 & Argentina & & Chile & & \\
\hline & Lepturus & 1 & & & Chile & & \\
\hline & Leymus & 1 & Argentina & & Chile & & \\
\hline & Lolium & 5 & Argentina & S Brasil & Chile & Paraguay & Uruguay \\
\hline & Megathyrsus & 1 & Argentina & S Brasil & Chile & Paraguay & \\
\hline & Melinis & 2 & Argentina & S Brasil & Chile & Paraguay & Uruguay \\
\hline & Miscanthus & 1 & & S Brasil & Chile & & Uruguay \\
\hline & Moorochloa & 1 & Argentina & & & & \\
\hline & Parapholis & 2 & Argentina & & Chile & & Uruguay \\
\hline & Piptatherum & 1 & Argentina & & Chile & & \\
\hline & Rhytachne & 1 & & S Brasil & & & \\
\hline & Rottboellia & 1 & Argentina & S Brasil & & & \\
\hline & Schedonorus & 1 & Argentina & & & & \\
\hline & Schismus & 2 & Argentina & & Chile & & \\
\hline & Sclerochloa & 1 & Argentina & & & & \\
\hline & Taeniatherum & 1 & & & Chile & & \\
\hline & Themeda & 1 & Argentina & S Brasil & & Paraguay & \\
\hline & Thinopyrum & 1 & Argentina & & & & \\
\hline \multirow[t]{4}{*}{ Polygonaceae } & Antigonon & 1 & Argentina & S Brasil & & Paraguay & \\
\hline & Emex & 1 & Argentina & & Chile & & Uruguay \\
\hline & Eriogonum & 1 & Argentina & & & & \\
\hline & Fallopia & 2 & & & Chile & & \\
\hline Primulaceae & Centunculus & 1 & Argentina & S Brasil & Chile & Paraguay & Uruguay \\
\hline Proteaceae & Grevillea & 1 & Argentina & & & & \\
\hline \multirow[t]{2}{*}{ Ranunculaceae } & Aquilegia & 1 & Argentina & & Chile & & \\
\hline & Ceratocephalus & 1 & Argentina & & & & \\
\hline Resedaceae & Reseda & 5 & Argentina & & Chile & & \\
\hline Rhamnaceae & Hovenia & 1 & Argentina & S Brasil & & Paraguay & \\
\hline \multirow[t]{6}{*}{ Rosaceae } & Cotoneaster & 4 & Argentina & & Chile & & \\
\hline & Crataegus & 1 & Argentina & & & & \\
\hline & Duchesnea & 1 & Argentina & S Brasil & Chile & & Uruguay \\
\hline & Eriobotrya & 1 & Argentina & S Brasil & & & \\
\hline & Malus & 2 & Argentina & & & & \\
\hline & Pyracantha & 2 & Argentina & & & & \\
\hline
\end{tabular}


Tabla 6. (Continuación).

\begin{tabular}{|c|c|c|c|c|c|c|c|}
\hline \multirow[t]{2}{*}{ Familia } & \multirow{2}{*}{$\begin{array}{l}\text { Género } \\
\text { Rosa }\end{array}$} & \multirow{2}{*}{$\begin{array}{c}\text { NoSp. }^{\circ} \text {. } \\
5\end{array}$} & \multicolumn{5}{|c|}{ Países } \\
\hline & & & Argentina & & Chile & & \\
\hline & Sanguisorba & 1 & Argentina & & Chile & & \\
\hline & Sorbus & 1 & Argentina & & & & \\
\hline & Spiraea & 2 & Argentina & S Brasil & & & \\
\hline \multirow[t]{2}{*}{ Rubiaceae } & Rubia & 1 & Argentina & & Chile & & \\
\hline & Sherardia & 1 & Argentina & & Chile & & Uruguay \\
\hline \multirow[t]{3}{*}{ Rutaceae } & Citrus & 2 & Argentina & S Brasil & & Paraguay & \\
\hline & Poncirus & 1 & Argentina & & & & \\
\hline & Ruta & 1 & Argentina & & Chile & & \\
\hline Salicaceae & Populus & 3 & Argentina & & Chile & & \\
\hline \multirow[t]{3}{*}{ Scrophulariaceae } & Myoporum & 1 & Argentina & & Chile & & Uruguay \\
\hline & Scrophularia & 2 & & S Brasil & Chile & & \\
\hline & Verbascum & 4 & Argentina & S Brasil & Chile & Paraguay & Uruguay \\
\hline Simaroubaceae & Ailanthus & 1 & Argentina & & Chile & & Uruguay \\
\hline Solanaceae & Brugmansia & 1 & Argentina & S Brasil & & Paraguay & \\
\hline Sphenocleaceae & Sphenoclea & 1 & Argentina & & & Paraguay & \\
\hline Tamaricaceae & Tamarix & 1 & Argentina & & & & \\
\hline Theaceae & Camellia & 1 & Argentina & & & & \\
\hline Thelypteridaceae & Macrothelypteris & 1 & Argentina & S Brasil & & Paraguay & Uruguay \\
\hline Ulmaceae & Ulmus & 1 & & & Chile & & \\
\hline Urticaceae & Soleirolia & 1 & & & Chile & & \\
\hline \multirow[t]{2}{*}{ Vitaceae } & Parthenocissus & 2 & Argentina & & & & \\
\hline & Vitis & 1 & Argentina & & Chile & & \\
\hline Zingiberaceae & Hedychium & 1 & Argentina & S Brasil & & Paraguay & \\
\hline Zygophyllaceae & Tribulus & 1 & Argentina & & Chile & & Uruguay \\
\hline
\end{tabular}

\section{Análisis florístico por país}

El mayor número de familias, géneros y especies se encuentra en la Argentina (2133 géneros y 10221 especies), país con mayor extensión geográfica en el Cono Sur (unos $2,790,000 \mathrm{~km}^{2}$, lo que representa el $60 \%$ del área total del Cono Sur, aproximadamente); le siguen el sur de Brasil con 1747 géneros y 9189 especies $\left(577,214 \mathrm{~km}^{2}\right)$, Paraguay, con 1356 géneros y 5296 especies $\left(406,635 \mathrm{~km}^{2}\right)$, Chile, con 1128 géneros y 5155 especies $\left(756,206 \mathrm{~km}^{2}\right)$ y Uruguay, con 985 géneros y 2911 especies $\left(175,016 \mathrm{~km}^{2}\right)$. Esta información por país está condensada en la Tabla 7 y representada en la Figura 1. El mayor número de especies endémicas de un país se verifica en Chile: 1962 especies, el $38 \%$ del total de especies de este país (Tabla 7).

En el Apéndice 2 se brinda un resumen de la flora vascular de cada país (Argentina, sur de Brasil, Chile, Paraguay y Uruguay), listando las familias presentes, el número de géneros y especies, discriminando entre endémicas, nativas e introducidas. (Apéndice 2 disponible en: http:// www.ojs.darwin.edu.ar/index.php/darwiniana/ article/view/861/1168). 
Tabla 7. Riqueza de especies en los distintos países del Cono Sur.

\begin{tabular}{|l|c|c|c|c|c|c|}
\hline País & Familias & Géneros & Especies & Endémicas & Nativas & Introducidas \\
\hline Argentina & 253 & 2133 & 10221 & 1731 & 7506 & 984 \\
\hline Sur de Brasil & 241 & 1747 & 9189 & 1215 & 7506 & 468 \\
\hline Chile & 187 & 1128 & 5155 & 1962 & 2487 & 706 \\
\hline Paraguay & 197 & 1356 & 5296 & 330 & 4796 & 170 \\
\hline Uruguay & 175 & 985 & 2911 & 106 & 2430 & 375 \\
\hline
\end{tabular}

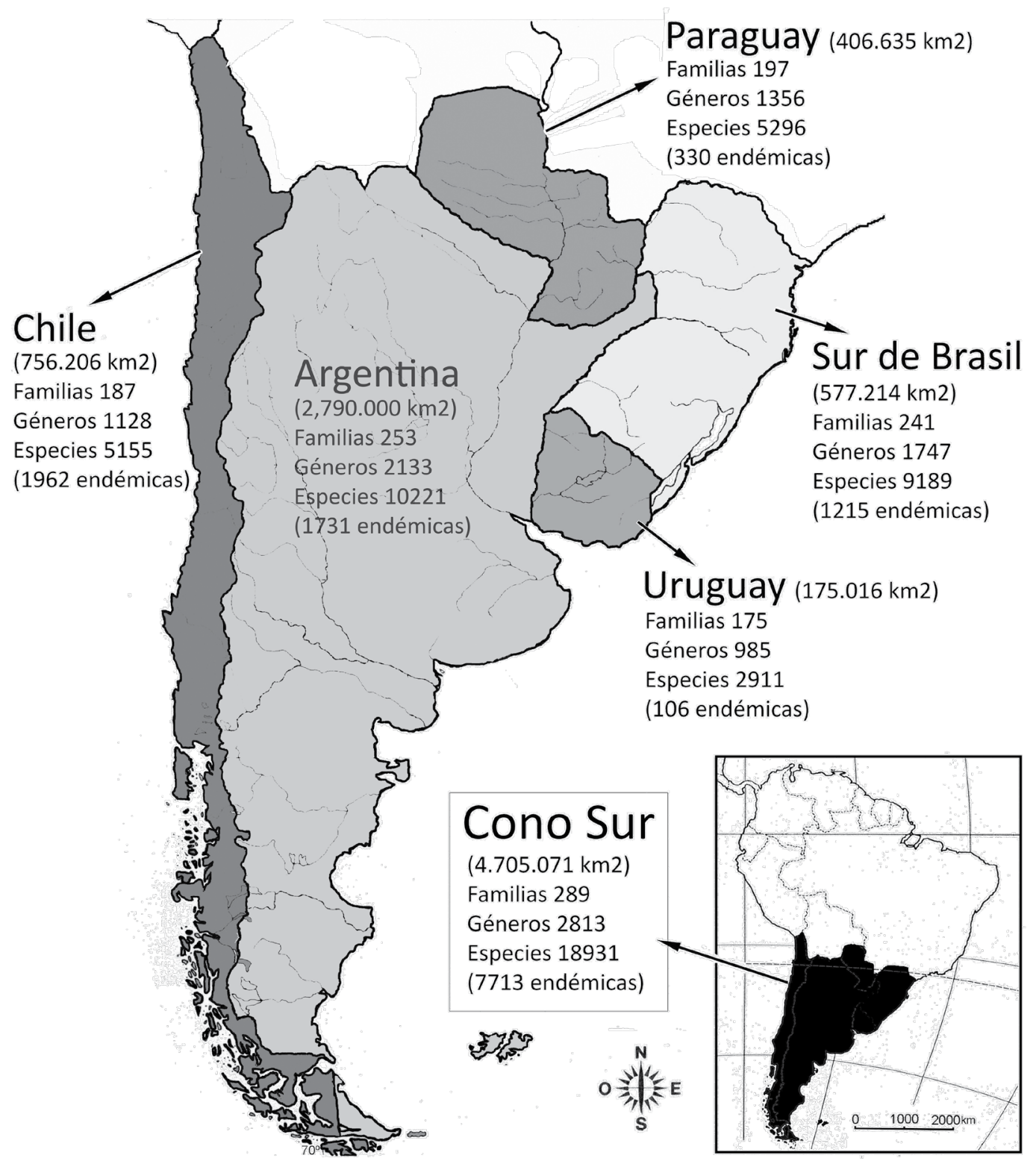

Figura 1. El Cono Sur y los países que lo integran: superficie, número de familias, géneros y especies (las endémicas entre paréntesis). 
Relaciones florísticas entre países

El análisis de las relaciones florísticas entre países (Tablas 8 y 9) y de las especies endémicas presentes (Tablas 10 y 11) muestra una gran afinidad entre Argentina y Chile, que comparten 1268 especies endémicas y exclusivas de estos dos países y, por otra parte, la del sur de Brasil con Uruguay, Paraguay y el este de la Argentina.

Tabla 8. Número de especies compartidas entre dos países.

\begin{tabular}{|c|c|c|c|c|c|}
\hline & Argentina & S. Brasil & Chile & Paraguay & Uruguay \\
\hline Argentina & & 3970 & 2703 & 3629 & 2537 \\
\hline S. Brasil & 3970 & & 593 & 3511 & 2371 \\
\hline Chile & 2703 & 593 & & 355 & 1653 \\
\hline Paraguay & 3629 & 3511 & 355 & & 1653 \\
\hline Uruguay & 2537 & 2371 & 651 & \\
\hline
\end{tabular}

Tabla 9. Número de especies compartidas en forma exclusiva entre dos países.

\begin{tabular}{|c|c|c|c|c|c|}
\hline & Argentina & S. Brasil & Chile & Paraguay & Uruguay \\
\hline Argentina & & 487 & 1961 & 722 & 164 \\
\hline S. Brasil & 487 & & 15 & 676 & 11 \\
\hline Chile & 1961 & 15 & & 1 & 4 \\
\hline Paraguay & 722 & 676 & 1 & & \\
\hline Uruguay & 164 & 189 & 11 & 4 & \\
\hline
\end{tabular}

Tabla 10. Número de especies endémicas del Cono Sur, compartidas por dos países.

\begin{tabular}{|c|c|c|c|c|c|}
\hline & Argentina & S. Brasil & Chile & Paraguay & Uruguay \\
\hline Argentina & & 659 & 1310 & 480 & 518 \\
\hline S. Brasil & 659 & & 23 & 381 & 530 \\
\hline Chile & 1310 & 23 & & 14 & 38 \\
\hline Paraguay & 480 & 381 & 14 & & 203 \\
\hline Uruguay & 518 & 530 & 38 & 203 & \\
\hline
\end{tabular}

Tabla 11. Número de especies endémicas del Cono Sur, compartidas en forma exclusiva por dos países.

\begin{tabular}{|c|c|c|c|c|c|}
\hline & Argentina & S. Brasil & Chile & Paraguay & Uruguay \\
\hline Argentina & & 107 & 1268 & 142 & 80 \\
\hline S. Brasil & 107 & & 0 & 56 & 0 \\
\hline Chile & 1268 & 0 & & 0 & 3 \\
\hline Paraguay & 142 & 56 & 0 & 3 & \\
\hline Uruguay & 80 & 117 & 0 & & \\
\hline
\end{tabular}




\section{CONCLUSIONES Y FUTURO}

La información actualizada de las plantas vasculares del Cono Sur demuestra la importancia de la flora subtropical y templada de América del Sur y su relevancia en lo que a la biodiversidad se refiere. Estos trabajos en ejecución resultan útiles tanto para proyectos internacionales de cooperación como nacionales. En primer término se puede citar la conexión, y colaboración, con emprendimientos similares en países vecinos del Cono Sur: la Lista do Brasil (BFG, 2018), la Flora del Paraguay (editada por L. Ramella en el Jardín Botánico de Ginebra), y el catálogo de las plantas vasculares de Chile (Rodríguez et al., 2018). A nivel mundial, estos proyectos participan de la iniciativa de la World Flora (www.worldfloraonline.org) y se asocian colecciones al GBIF (Global Biodiversity Information Facility, (www.gbif.org) o de investigaciones de las plantas vasculares del Nuevo Mundo (Ulloa et al., 2017).

Los resultados obtenidos proveen una base robusta y predecible para: $a$ ) investigaciones en taxonomía, mediante el acceso a descripciones, colecciones de herbario, fotografías, ilustraciones, mapas de distribución, etc., a través del portal web del Catálogo; b) trabajos florísticos a diferentes escalas; en este sentido, la información disponible puede utilizarse, y ha sido utilizada exitosamente, para el desarrollo de manuales o guías de campo, de gran utilidad para múltiples usuarios; c) toma de decisiones, por cuanto los datos disponibles representan una herramienta indispensable para los usuarios de la tierra en regiones subtropicales y templadas, y para la gestión y conservación de la biodiversidad. Tal como se cita en trabajos previos (BFG, 2018), el ejemplo de la lista de plantas de Sudáfrica (Crouch \& Smith, 2011), muestra como una lista nacional de plantas, o regional, puede ser la base de iniciativas socioeconómicas aplicadas.

Finalmente, deseamos destacar la importancia de mantener la información florística y taxonómica actualizada y mejorada constantemente. El compendio, interpretación y articulación de esta enorme cantidad de datos constituye una base sólida para conocer la biodiversidad de plantas vasculares del Cono Sur, indispensable para trazar estrategias de desarrollo sustentable y conservación de especies.

\section{BIBLIOGRAFÍA}

BFG -The Brazil Flora Group. 2018. Brazilian Flora 2020: Innovation and collaboration to meet Target 1 of the Global Strategy for Plant Conservation (GSPC). Rodriguésia 69(4): 1513-1527. DOI: 10.1590/2175-7860201869402

Brummitt, R. K. \& C. E. Powell. 1992. Authors of Plant Names. Royal Botanic Gardens, Kew.

Byng, J. W., M. W. Chase, M. J. M. Christenhusz, M. F. Fay, W. S. Judd., A. N. Sennikov, D. E. Soltis, P. S. Soltis \& P. F. Stevens. 2016. An update of the Angiosperm Phylogeny Group classification for the orders and families of flowering plants: APG IV. Botanical Journal of the Linnean Society 181: 1-20.

Crouch, N. R. \& G. F. Smith. 2011. Informing and influencing the interface between biodiversity science and biodiversity policy in South Africa. Botanical Journal of the Linnean Society 166: 301-309.

Rodríguez, R., Marticorena, C., Alarcón, D., Baeza, C., Cavieres, L., Finot, V. L., Fuentes, N., Kiessling, A., Mihoc, M., Pauchard, A., Ruiz, E., Sanchez, P. \& Marticorena, A. 2018. Catálogo de las plantas vasculares de Chile. Gayana, Botanica 75(1): 1-430.

Thiers, B. [permanentemente actualizado, consulta 2019] Index Herbariorum: a global directory of public herbaria and associated staff. New York Botanical Garden's Virtual Herbarium, http://sweetgum.nybg.org/ih

Turland, N. J., J. H. Wiersema, F. R. Barrie, W. Greuter, D.L. Hawksworth, P.S. Herendeen, S. Knapp, W. H. Kusber, D.-Z. Li, K. Marhold, T. W. May, J. McNeill, A.M. Monro, J. Prado, M.J. Price, and G.F. Smith (eds.). 2018. International Code of Nomenclature for algae, fungi, and plants (Shenzhen Code) adopted by the Nineteenth International Botanical Congress Shenzhen, China, July 2017. Regnum Vegetabile 159. Koeltz Botanical Books, Glashütten.

Ulloa Ulloa, C., Acevedo-Rodríguez, P., Beck, S., Belgrano, M. J., Bernal, R., Berry, P. E., Brako, L., Celis, M., Davidse, G., Forzza, R., Gradstein, R., Hokche, O., León, B., LeónYáñez, S., Magill, R. E., Neill, D. A., Nee, M., Raven, P. H., Stimmel, H., Strong, M. T., Villaseñor, J. L., Zarucchi, J. L., Zuloaga, F. O. \& Jorgensen, P. M. 2017. How many vascular plant species occur in the New World. Science 358, 6370: 1614-1617. DOI: 10.1126/science.aao0398

Zuloaga, F. O., E. G. Nicora, Z. E. Rúgolo de Agrasar, O. Morrone, J. F. Pensiero \& A. M. Cialdella. 1994. Catálogo de la familia Poaceae en la República Argentina. Monographs in Systematic Botany from the Missouri Botanical Garden 47: 1-178. 
DARWINIANA, nueva serie 7(2): 208-278. 2019

Zuloaga, F. O.\& O. Morrone (eds.). 1996. Catálogo de las Plantas Vasculares de la Argentina: Pteridophyta, Gymnospermae y Monocotyledoneae (excluyendo Poaceae). Monographs in Systematic Botany from the Missouri Botanical Garden 60: $1-332$.

Zuloaga, F. O. \& O. Morrone (eds.). 1999. Catálogo de las Plantas Vasculares de la Argentina: Dicotyledoneae. Monographs in Systematic Botany from the Missouri Botanical Garden 74: 1-1246.
Zuloaga, F. O., Morrone, O. \& M. J. Belgrano. 2008 (eds.). Catálogo de las plantas vasculares del Cono Sur (Argentina, sur de Brasil, Chile, Paraguay y Uruguay). Monographs in Systematic Botany from the Missouri Botanical Garden 107: 1-3348.

Zuloaga, F. O. \& M. J. Belgrano. 2015. The Catalogue of Vascular Plants of the Southern Cone and the Flora of Argentina: their contribution to the World Flora. Rodrigúesia 66(4): 989-1024. 